\title{
Quasilinear elliptic equations with a source reaction term involving the function and its gradient and measure data
}

\author{
Marie-Françoise Bidaut-Véron* \\ Quoc-Hung Nguyen ${ }^{\dagger}$ \\ Laurent Véron ${ }^{\ddagger}$
}

\begin{abstract}
We study the equation $-\operatorname{div}(A(x, \nabla u))=|u|^{q_{1}-1} u|\nabla u|^{q_{2}}+\mu$ where $A(x, \nabla u) \sim|\nabla u|^{p-2} \nabla u$ in some suitable sense, $\mu$ is a measure and $q_{1}, q_{2}$ are nonnegative real numbers and satisfy $q_{1}+q_{2}>p-1$. We give sufficient conditions for existence of solutions expressed in terms of the Wolff potential or the Riesz potentials of the measure. Finally we connect the potential estimates on the measure with Lipchitz estimates with respect to some Bessel or Riesz capacity.

key-words: Quasilinear equations; Wolff and Riesz potentials; Hardy-Littlewood maximal function; renormalized solutions; Bessel and Riesz capacities.
\end{abstract}

2010 Mathematics Subject Classication: 31C15, 35J62, 35J92, 35R06, 45 G15.

\section{Contents}

1 Introduction and main results 1

2 Estimates on potential $\quad 6$

3 Renormalized solutions $\quad 30$

4 Proof of the main results

\section{Introduction and main results}

This article is devoted to the study of existence of solutions of some second order quasilinear equations with measure data with a source-reaction term involving the function and its gradient. First we consider the problem with a Radon measure $\mu$ in $\mathbb{R}^{N}$ in the whole space

$$
-\operatorname{div}(A(x, \nabla u))=|u|^{q_{1}-1} u|\nabla u|^{q_{2}}+\mu \text { in } \mathbb{R}^{N} .
$$

*E-mail address: veronmf@univ-tours.fr, Laboratoire de Mathématiques et Physique Théorique,Université François Rabelais, Tours, France

${ }^{\dagger}$ E-mail address: qhnguyen@shanghaitech.edu.cn, ShanghaiTech University, 393 Middle Huaxia Road, Pudong, Shanghai, 201210, China.

${ }^{\ddagger}$ E-mail address: Laurent.Veron@lmpt.univ-tours.fr, Laboratoire de Mathématiques et Physique Théorique,Université François Rabelais, Tours, France 
In this setting, $(x, \xi) \mapsto A(x, \xi)$ from $\mathbb{R}^{N} \times \mathbb{R}^{N}$ to $\mathbb{R}^{N}$ is a Carathéodory vector field satisfying for almost all $x \in \mathbb{R}^{N}$ the growth and ellipticity conditions

$$
\begin{aligned}
& |\mathcal{A}(x, \xi)|+\left.|\xi|\left|\nabla_{\xi} \mathcal{A}(x, \xi)\right|\left|\leq \Lambda_{1}\right| \xi\right|^{p-1} \quad \text { for all } \xi \in \mathbb{R}^{N}, \\
& \langle\mathcal{A}(x, \xi)-\mathcal{A}(x, \eta), \xi-\eta\rangle \geq \Lambda_{2}\left(|\xi|^{2}+|\eta|^{2}\right)^{\frac{p-2}{2}}|\xi-\eta|^{2} \text { for all } \xi, \eta \in \mathbb{R}^{N}, \\
& |A(x, \xi)-A(y, \xi)| \leq \Lambda_{1}|x-y|^{\alpha_{0}}|\xi|^{p-1} \text { for all } \xi \in \mathbb{R}^{N} \\
& A(x, \lambda \xi)=|\lambda|^{p-2} \lambda A(x, \xi) \quad \text { for all }(\lambda, \xi) \in \mathbb{R} \times \mathbb{R}^{N}
\end{aligned}
$$

where $\Lambda_{1} \geq \Lambda_{2}>0$ are constants and $\frac{3 N-2}{2 N-1}<p<N$, and where $q_{1}, q_{2}>0$ satisfy $q_{1}+q_{2}>p-1$, and $\alpha_{0} \in(0,1)$. The special case $\mathcal{A}(x, \xi)=|\xi|^{p-2} \xi$ gives rise to the standard $p$-Laplacian $\Delta_{p} u=\operatorname{div}\left(|\nabla u|^{p-2} \nabla u\right)$. Note that these conditions imply that $A(x, 0)=0$ for a.e. $x \in \mathbb{R}^{N}$, and

$$
\left\langle\nabla_{\xi} A(x, \xi) \lambda, \eta\right\rangle \geq 2^{\frac{p-2}{2}} \Lambda_{2}|\xi|^{p-2}|\eta|^{2}
$$

for every $(\eta, \xi) \in \mathbb{R}^{N} \times \mathbb{R}^{N} \backslash\{(0,0)\}$ and a.e. $x \in \mathbb{R}^{N}$.

When $p=2, q_{1}=q_{2}=1$, we obtain a toy model of the forced stationary NavierStokes equations describing the motion of incompressible fluid in the whole space $\mathbb{R}^{N}$ :

$$
\begin{aligned}
-\Delta U+\nabla p & =-U \cdot \nabla U+F, \\
\operatorname{div}(U) & =0,
\end{aligned}
$$

in $\mathbb{R}^{N}$,where $U=\left(U_{1}, \ldots, U_{N}\right): \mathbb{R}^{N} \rightarrow \mathbb{R}^{N}$ is an unknown velocity of the fluid, $P: \mathbb{R}^{N} \rightarrow \mathbb{R}$ is an unknown pressure, and $F=\left(F_{1}, \ldots, F_{N}\right): \mathbb{R}^{N} \rightarrow \mathbb{R}^{N}$ is a given external force.

We also consider the homogeneous Dirichlet problem with measure data in a bounded domain $\Omega \subset \mathbb{R}^{N}$

$$
\begin{aligned}
-\operatorname{div}(A(x, \nabla u)) & =|u|^{q_{1}-1} u|\nabla u|^{q_{2}}+\mu & & \text { in } \Omega, \\
u & =0 & & \text { on } \partial \Omega,
\end{aligned}
$$

where, in this setting, $A:(x, \xi) \mapsto A(x, \xi)$ is a Carathéodory vector field defined in $\Omega \times \mathbb{R}^{N}$ satisfying (1.2) (i)-(iv) in $\Omega \times \mathbb{R}^{N}$, and $q_{1}, q_{2}$ are as in the first case; and $\Omega \subset \mathbb{R}^{N}$ is a bounded domain with a $C^{1, \beta_{0}}$ boundary for $\beta_{0} \in(0,1)$ and such that $\Omega \subset B_{R}\left(x_{0}\right)$ for some $R>0$ and $x_{0} \in \Omega$. The two specific cases,

$$
\begin{aligned}
-\operatorname{div}(A(x, \nabla u)) & =|u|^{q_{1}-1} u+\mu & & \text { in } \Omega \\
u & =0 & & \text { in } \partial \Omega,
\end{aligned}
$$

and

$$
\begin{aligned}
-\operatorname{div}(A(x, \nabla u)) & =|\nabla u|^{q_{2}}+\mu & & \text { in } \Omega, \\
u & =0 & & \text { in } \partial \Omega,
\end{aligned}
$$

have been studied thoroughly in the last decade. Each of these equations carries a critical exponent $q_{j}^{c}: \quad q_{1}^{c}=\frac{N(p-1)}{N-p}$ for equation (1.5) and $q_{2}^{c}=\frac{N(p-1)}{N-1}$ for (1.6). These critical thresholds mean that if $0<p-1<q_{1}<q_{1}^{c}$ for (1.5) and $1-\frac{1}{N}<p-1<q_{1}<q_{1}^{c}$ for (1.6) any nonnegative bounded measure is eligible for the respective equation, provided it is small enough. Concerning equation (1.1), the criticality is expressed by a linear relation $0<q_{1}(N-p)+q_{2}(N-1)<N(p-1)$. Then, if $p>2-\frac{1}{N}$ and $q_{1}+q_{2}>p-1$, problem (1.4) any Radon measure small enough, see [27, Chap 6-2] and references therein. The treatment of the supercritical case for equations (1.5) and (1.6) have been treated more 
recently. In these cases not only the measure $\mu$ has to be small enough, but also it cannot be too concentrated with respect to some Bessel capacity, specific to each problem. It is proved in [22] that if $\mu$ is a nonnegative Radon measure with compact support in $\Omega$, a necessary and sufficient condition for the existence of a renormalized solution to (1.5) is that there exists some $c_{1}>0$ depending on the structural constants and $\|\mu\|_{\mathfrak{M}}$ such that

$$
\mu(K) \leq c_{1} \operatorname{Cap}_{\mathbf{G}_{p}, \frac{q_{1}}{q_{1}+1-p}}(K) \text { for all compact set } K \subset \bar{\Omega},
$$

where $\operatorname{Cap}_{\mathbf{G}_{p}, \frac{q_{1}}{q_{1}+1-p}}$ denotes some Bessel capacity. Concerning (1.6), assuming $\max \{p-$ $1,1\}<q_{2}$, it is proved in $[23,24,16,19,18]$ that there exists a structural constant $c_{2}>0$ as above such that if

$$
|\mu|(K) \leq c_{2} \operatorname{Cap}_{\mathbf{G}_{1}, \frac{q_{2}}{q_{2}+1-p}}(K) \text { for all compact set } K \subset \Omega,
$$

there exists a renormalized solution to (1.6) with the property that

$$
\int_{K}|\nabla u|^{q_{2}} d x \leq c_{3} \operatorname{Cap}_{\mathbf{G}_{1}, \frac{q_{2}}{q_{2}+1-p}}(K) \text { for all compact set } K \subset \bar{\Omega},
$$

for some $c_{3}>0$.

The complete expression of these results as well as the ones we will state below necessitates the introduction of several definitions and notations from harmonic analysis such as Wolff potential, Riesz potentials, Bessel spaces and maximal functions. The role of these operators has appeared to be a key-stone for conducting a fine analysis of quasilinear equations with measure data; this is very clearly presented in the introduction of the seminal paper [22]. If $D$ is either a bounded domain or whole $\mathbb{R}^{N}$, we denote by $\mathfrak{M}(D)$ (resp. $\mathfrak{M}_{b}(D)$ ) the set of Radon measures (resp. bounded Radon measures) in $D$. Their positive cones are $\mathfrak{M}^{+}(D)$ and $\mathfrak{M}_{b}^{+}(D)$ respectively. For $R \in(0, \infty]$, we define the $R$ - truncated Wolff potential $\mathbf{W}_{\alpha, p}^{R}(\alpha \in(0, N / p), p>1)$ and the $R$-truncated Riesz potential $\mathbf{I}_{\beta}^{R}(\beta \in(0, N))$ of a measure $\mu \in \mathfrak{M}^{+}\left(\mathbb{R}^{N}\right)$ by

$$
\mathbf{W}_{\alpha, p}^{R}[\mu](x)=\int_{0}^{R}\left(\frac{\mu\left(B_{\rho}(x)\right)}{\rho^{N-\alpha p}}\right)^{\frac{1}{p-1}} \frac{d \rho}{\rho} \text { and } \mathbf{I}_{\beta}^{R}[\mu](x)=\int_{0}^{R} \frac{\mu\left(B_{\rho}(x)\right)}{\rho^{N-\beta}} \frac{d \rho}{\rho},
$$

for all $x$ in $\mathbb{R}^{N}$. If $R=\infty$, we drop it in the expressions of (1.10). We write $\mathbf{W}_{\alpha, p}^{R}[f], \mathbf{I}_{\beta}^{R}[f]$ in place of $\mathbf{W}_{\alpha, p}^{R}[\mu], \mathbf{I}_{\beta}^{R}[\mu]$ whenever $d \mu=f d x$, where $f \in L_{l o c}^{1}\left(\mathbb{R}^{N}\right)$.

For $\alpha>0, p>1$, the $\left(\mathbf{I}_{\alpha}, p\right)$-capacity, $\left(\mathbf{G}_{\alpha}, p\right)$-capacity of a Borel set $O \subset \mathbb{R}^{N}$ are defined by

$$
\begin{aligned}
& \operatorname{Cap}_{\mathbf{I}_{\alpha}, p}(O)=\inf \left\{\int_{\mathbb{R}^{N}}|g|^{p} d x: g \in L_{+}^{p}\left(\mathbb{R}^{N}\right), \mathbf{I}_{\alpha}[g] \geq \chi_{O}\right\}, \\
& \operatorname{Cap}_{\mathbf{G}_{\alpha}, p}(O)=\inf \left\{\int_{\mathbb{R}^{N}}|g|^{p} d x: g \in L_{+}^{p}\left(\mathbb{R}^{N}\right), \mathbf{G}_{\alpha} * g \geq \chi_{O}\right\},
\end{aligned}
$$

where $\mathbf{G}_{\alpha}=\mathcal{F}^{-1}\left(\left(1+|\xi|^{2}\right)^{-\frac{\alpha}{2}}\right)$ is the Bessel kernel of order $\alpha$, see [1] (and $\mathcal{F}$ and $\mathcal{F}^{-1}$ are respectively the Fourier transform and its inverse).

The results we prove consist in obtaining sufficient conditions for the solvability of (1.1) or (1.4) where $A$ is of the form (1.2) in $\mathbb{R}^{N}$ (or $\Omega$ ) expressed in terms of inequalities between 
Wolff or Riesz potentials of $\mu$. In order to obtain these inequalities we will develop a series of sharp relations between these potentials and will connect them with some specific capacities. We recall that a Radon measure $\mu$ in $\mathbb{R}^{N}$ (or $\Omega$ ) is absolutely continuous with respect to some capacity Cap in $\mathbb{R}^{N}$ (or $\Omega$ ) if for a Borel set $E$

$$
\operatorname{Cap}(E)=0 \Longrightarrow|\mu|(E)=0
$$

and it is Lipschitz continuous (with constant $c>0$ ) if

$$
|\mu|(E) \leq c \operatorname{Cap}(E) \quad \text { for all Borel set } E \text {. }
$$

The capacity associated to the Sobolev space $W^{1, p}\left(\mathbb{R}^{N}\right)$ is denoted by $\operatorname{Cap}_{1, p}$. It coincides with $\mathrm{Cap}_{\mathbf{G}_{1}, p}$, [1, Th 1.2.3]. A measure is called diffuse if it is absolutely continuous with respect to $\mathrm{Cap}_{1, p}$

Our first result deals with the equation in the whole space,

Theorem 1.1 Let $q_{1}, q_{2}>0, q_{1}+q_{2}>p-1,0<q_{2}<\frac{N(p-1)}{N-1}$ and $\mu \in \mathfrak{M}\left(\mathbb{R}^{N}\right)$. Assume that $A(x, \xi)=A(\xi)$ for any $(x, \xi) \in \mathbb{R}^{N} \times \mathbb{R}^{N}$. If for some $C>0$ depending on $p, N, q_{j}$ and $\Lambda_{j}(j=1,2)$, there holds

$$
|\mu|(K) \leq C \operatorname{Cap}_{\mathbf{I}_{\frac{q_{1} p+q_{2}}{q_{1}+q_{2}}}, \frac{q_{1}+q_{2}}{q_{1}+q_{2}-p+1}}(K) \quad \text { for all compact } K \subset \mathbb{R}^{N},
$$

then problem (1.1) admits a distributional solution $u$ which satisfies

$$
|u| \leq C_{0} \mathbf{W}_{1, p}[|\mu|], \quad|\nabla u| \leq C_{0} \mathbf{W}_{\frac{1}{p}, p}[|\mu|]
$$

if $p>2$, and

$$
|u| \leq C_{0}\left(\mathbf{I}_{p}[|\mu|]\right)^{\frac{1}{p-1}}, \quad|\nabla u| \leq C_{0}\left(\mathbf{I}_{1}[|\mu|]\right)^{\frac{1}{p-1}},
$$

if $\frac{3 N-2}{2 N-1}<p \leq 2$. Moreover, if $\mu \geq 0$, then $u \geq 0$.

Notice also that if $\mu \geq 0$ the solutions $u$ in Theorem 1.1 are nonnegative p-super-harmonic functions.

When $\mathbb{R}^{N}$ is replaced by a bounded domain $\Omega$, we have the following general result.

Theorem 1.2 Let $q_{1}, q_{2}>0, q_{1}+q_{2}>p-1,0<q_{2}<\frac{N(p-1)}{N-1}$. Let $\mu \in \mathfrak{M}(\Omega)$ be such that $\operatorname{dist}(\operatorname{supp}(\mu), \partial \Omega)>0$. If for some $C>0$ depending on $p, N, q_{j}$ and $\Lambda_{j}(j=1,2), \Omega$ and dist $(\operatorname{supp}(\mu), \partial \Omega)$ there holds

$$
|\mu|(K) \leq C \operatorname{Cap}_{\mathbf{G}_{\frac{q_{1} p+q_{2}}{q_{1}+q_{2}}}, \frac{q_{1}+q_{2}}{q_{1}+q_{2}-p+1}}(K) \quad \text { for all compact } K \subset \mathbb{R}^{N},
$$

then problem (1.4) admits a renormalized solution u satisfying

$$
|u| \leq C_{0} \mathbf{W}_{1, p}[|\mu|] \quad \text { and }|\nabla u| \leq C_{0} \mathbf{W}_{\frac{1}{p}, p}[|\mu|]
$$

if $p>2$, and

$$
|u| \leq C_{0}\left(\mathbf{I}_{p}[|\mu|]\right)^{\frac{1}{p-1}}, \quad|\nabla u| \leq C_{0}\left(\mathbf{I}_{1}[|\mu|]\right)^{\frac{1}{p-1}},
$$

if $\frac{3 N-2}{2 N-1}<p \leq 2$. Moreover, if $\mu \geq 0$, then $u \geq 0$. 
The key-stone of our method which combines sharp potential estimates and Schauder fixed point theorem is to reduce our problems (1.1)-(1.4) to a system of nonlinear Wolff integral equations in the spirit of the method developed in [8] and [20]. The proof of Theorem 1.1 (and similarly for Theorem1.2) is based upon the existence of a fixed point obtained by Schauder's theorem, of the mapping $S$ which associates to

$$
v \in E_{\Lambda}=\left\{v:|v| \leq \Lambda\left(\mathbf{I}_{p}\left[\left|\mu_{n, k}\right|\right]\right)^{\frac{1}{p-1}} \text { s.t. }|\nabla v| \leq \Lambda\left(\mathbf{I}_{1}\left[\left|\mu_{n, k}\right|\right]\right)^{\frac{1}{p-1}} \text { in } B_{2 k}\right\}
$$

the solution $u=u_{n, k}=S(v)$, of

$$
\begin{aligned}
-\operatorname{div}(A(x, \nabla u)) & =\chi_{B_{k}}|v|^{q_{1}-1} v|\nabla v|^{q_{2}}+\mu_{n, k} & & \text { in } B_{2 k} \\
u & =0 & & \text { on } \partial B_{2 k}
\end{aligned}
$$

where $\mu_{n, k}$ is a smooth approximation of $\mu$ with support in $B_{k}:=B_{k}(0)$ and $\Lambda>0, k$, $n \in \mathbb{N}_{*}$ are parameters. In order to prove that the set $E_{\Lambda}$ is invariant under $S$ we use a series of a priori estimates dealing with renormalized solutions of

$$
\begin{aligned}
-\operatorname{div}(A(x, \nabla u)) & =\nu & & \text { in } B_{2 k}, \\
u & =0 & & \text { on } \partial B_{2 k},
\end{aligned}
$$

where $\nu \in \mathfrak{M}_{b}(\Omega)$, and for our purpose $\nu=\chi_{B_{k}(0)}|v|^{q_{1}-1} v|\nabla v|^{q_{2}}+\mu_{n, k}$. Then we use pointwise estimates satisfied by a renormalized solution, e.g. in the case $p \leq 2$, there holds a.e. in $B_{k}$

$$
|u(x)| \leq C\left(\mathbf{I}_{p}\left[\left.\left|\chi_{B_{k}}\right| v\right|^{q_{1}-1} v|\nabla v|^{q_{2}}+\mu_{n, k} \mid\right](x)\right)^{\frac{1}{p-1}}
$$

and

$$
|\nabla u(x)| \leq C\left(\mathbf{I}_{1}\left[\left.\left|\chi_{B_{k}}\right| v\right|^{q_{1}-1} v|\nabla v|^{q_{2}}+\mu_{n, k} \mid\right](x)\right)^{\frac{1}{p-1}},
$$

see Theorem 3.6 and Corollary 3.8.

Using the fact that $v \in E_{\Lambda}$ we derive

$$
|u(x)| \leq C\left(\Lambda^{q_{1}+q_{2}} \mathbf{I}_{p}\left[\left(\mathbf{I}_{p}\left[\left|\mu_{n, k}\right|\right]\right)^{\frac{q_{1}}{p-1}}\left(\mathbf{I}_{1}\left[\left|\mu_{n, k}\right|\right]\right)^{\frac{q_{2}}{p-1}}\right](x)+\mathbf{I}_{p}\left[\left|\mu_{n, k}\right|\right](x)\right)^{\frac{1}{p-1}}
$$

and

$$
|\nabla u(x)| \leq C\left(\Lambda^{q_{1}+q_{2}} \mathbf{I}_{1}\left[\left(\mathbf{I}_{p}\left[\left|\mu_{n, k}\right|\right]\right)^{\frac{q_{1}}{p-1}}\left(\mathbf{I}_{1}\left[\left|\mu_{n, k}\right|\right]\right)^{\frac{q_{2}}{p-1}}\right](x)+\mathbf{I}_{1}\left[\left|\mu_{n, k}\right|\right](x)\right)^{\frac{1}{p-1}} .
$$

At this point we use the multplicative inequalities concerning the Riesz potential provided the measures satisfies some Lipschitz continuity estimate with respect to $\operatorname{Cap}_{\mathbf{I}_{\frac{p q_{1}+\beta p q_{2}}{q_{1}+q_{2}}, \frac{q_{1}+q_{2}}{q_{1}+q_{2}+1-p}}}$ :

$$
\mathbf{I}_{\alpha}\left[\left(\mathbf{I}_{p}\left[\left|\mu_{n, k}\right|\right]\right)^{\frac{q_{1}}{p-1}}\left(\mathbf{I}_{1}\left[\left|\mu_{n, k}\right|\right]\right)^{\frac{q_{2}}{p-1}}\right] \leq M \mathbf{I}_{\alpha}\left[\left|\mu_{n, k}\right|\right] \quad \text { for } \alpha=1 \text { or } p .
$$

These multiplicative inequalities are the key of our construction, since they imply that for suitable choice of $\Lambda, E_{\Lambda}$ is invariant. The compactness of $S$ being easy to prove we derive the existence of a solution to (1.19).

One of the tools is a series of equivalence linking the Lipschitz continuity of $\mu$ with respect to some capacity with integral estimates of the Wolff (or Riesz) potential of the measure and even to a system of nonlinear Wolff integral equations as in [20]. In this spirit we prove the following: 
Theorem 1.3 Let $1<p<N, 0<\beta<1, q_{1}, q_{2}>0$ such that $q_{1}+q_{2}>p-1$ and $q_{2}<\frac{N(p-1)}{N-\beta p}$. If $\mu$ is a nonnegative measure in $\mathbb{R}^{N}$, the following statements are equivalent: (a) The inequality

$$
\mu(K) \leq C_{1} \operatorname{Cap}_{I_{\frac{p q_{1}+\beta p q_{2}}{q_{1}+q_{2}}}, \frac{q_{1}+q_{2}}{q_{1}+q_{2}+1-p}}(K),
$$

holds for any compact set $K \subset \mathbb{R}^{N}$, for some $C_{1}>0$.

(b) The inequality

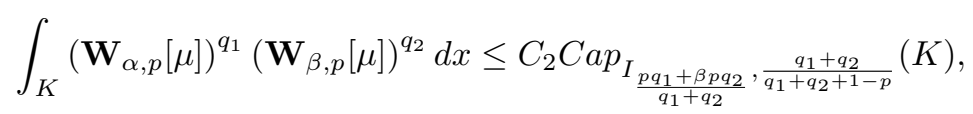

holds for any compact set $K \subset \mathbb{R}^{N}$ and some $C_{2}>0$.

(c) The inequalities

$$
\begin{aligned}
& \mathbf{W}_{1, p}\left[\left(\mathbf{W}_{1, p}[\mu]\right)^{q_{1}}\left(\mathbf{W}_{\frac{1}{p}, p}[\mu]\right)^{q_{2}}\right] \leq C_{3} \mathbf{W}_{1, p}[\mu] \\
& \mathbf{W}_{\frac{1}{p}, p}\left[\left(\mathbf{W}_{1, p}[\mu]\right)^{q_{1}}\left(\mathbf{W}_{\frac{1}{p}, p}[\mu]\right)^{q_{2}}\right] \leq C_{3} \mathbf{W}_{\frac{1}{p}, p}[\mu],
\end{aligned}
$$

in $\mathbb{R}^{N}$ are verified for some $C_{3}>0$.

(d) The system of equations

$$
\begin{aligned}
& U=\mathbf{W}_{1, p}\left[U^{q_{1}} V^{q_{2}}\right]+\epsilon \mathbf{W}_{1, p}[\mu] \\
& V=\mathbf{W}_{\frac{1}{p}, p}\left[U^{q_{1}} V^{q_{2}}\right]+\epsilon \mathbf{W}_{\frac{1}{p}, p}[\mu],
\end{aligned}
$$

in $\mathbb{R}^{N}$ has a solution $U, V \geq 0$ for $\epsilon>0$ small enough.

Actually the full statement is more complete and the above Theorem is a consequence of Theorem 2.10. Furthermore it has an analogue in $\Omega$, see Theorem 2.12.

\section{Estimates on potential}

In the sequel $C$ denotes a generic constant depending essentially on some structural constants (i.e. the ones associated to the operator and reaction term) and the domain, the value of which may change from one occurence to another. Sometimes, in order to avoid confusion, we introduce notations $C_{j}, j=0,1,2 \ldots$. We also use the notation $\asymp$ to assert that the two quantities linked by this relation are comparable up to multiplication by constants of the previous type. The following result is a general version of results of Phuc and Verbitsky $[22, \mathrm{Th} 2.3]$. It connects the Lipschitz continuity of a positive measure in $\mathbb{R}^{N}$ with respect to some Riesz capacity to various integral or pointwise estimates of Wolff potentials of this measure.

Theorem 2.1 Let $1<p<N / \alpha, q>p-1, \mu \in \mathfrak{M}^{+}\left(\mathbb{R}^{N}\right)$. Then, the following statements are equivalent:

(a) The inequality

$$
\mu(K) \leq C_{1} \operatorname{Cap}_{\mathbf{I}_{\alpha p}, \frac{q}{q-p+1}}(K),
$$

holds for any compact set $K \subset \mathbb{R}^{N}$, for some $C_{1}>0$. 
(b) The inequality

$$
\int_{K}\left(\mathbf{W}_{\alpha, p}[\mu](y)\right)^{q} d y \leq C_{2} \operatorname{Cap}_{\mathbf{I}_{\alpha p, \frac{q}{q-p+1}}}(K),
$$

holds for any compact set $K \subset \mathbb{R}^{N}$, for some $C_{2}>0$.

(c) The inequality

$$
\int_{\mathbb{R}^{N}}\left(\mathbf{W}_{\alpha, p}\left[\chi_{B_{t}(x)} \mu\right](y)\right)^{q} d y \leq C_{3} \mu\left(B_{t}(x)\right),
$$

holds for any $x \in \mathbb{R}^{N}$ and $t>0$, for some $C_{3}>0$.

(d) The inequality

$$
\mathbf{W}_{\alpha, p}\left[\left(\mathbf{W}_{\alpha, p}[\mu]\right)^{q}\right] \leq C_{4} \mathbf{W}_{\alpha, p}[\mu]<\infty,
$$

holds almost everywhere in $\mathbb{R}^{N}$, for some $C_{4}>0$.

Proof. Step 1: Proof of (a) $\Leftrightarrow$ (b). By [12, Theorem 1.1], (see also [7, Theorem 2.3]) we have

$$
\int_{\mathbb{R}^{N}}\left(\mathbf{I}_{\alpha p}[\nu](y)\right)^{\frac{q}{p-1}} w(y) d y \asymp \int_{\mathbb{R}^{N}}\left(\mathbf{W}_{\alpha, p}[\nu](y)\right)^{q} w(y) d y \text { for all } \nu \in \mathfrak{M}^{+}\left(\mathbb{R}^{N}\right),
$$

where $w$ belongs to the Muckenhoupt class $\mathbf{A}_{\infty}$. So, thanks to [21, Lemma 3.1] we obtain

$$
\sup _{K \in \mathcal{K}\left(\mathbb{R}^{N}\right)} \frac{\int_{K}\left(\mathbf{I}_{\alpha p}[\nu](y)\right)^{\frac{q}{p-1}} d y}{\operatorname{Cap}_{\mathbf{I}_{\alpha p}, \frac{q}{q-p+1}}(K)} \asymp \sup _{K \in \mathcal{K}\left(\mathbb{R}^{N}\right)} \frac{\int_{K}\left(\mathbf{W}_{\alpha, p}[\nu](y)\right)^{q} d y}{\operatorname{Cap}_{\mathbf{I}_{\alpha p}, \frac{q}{q-p+1}}(K)} \quad \text { for all } \nu \in \mathfrak{M}^{+}\left(\mathbb{R}^{N}\right),
$$

where $\mathcal{K}\left(\mathbb{R}^{N}\right)$ denotes the set of compact subsets of $\mathbb{R}^{N}$. Moreover, by [21, Theorem 2.1],

$$
\sup _{K \in \mathcal{K}\left(\mathbb{R}^{N}\right)} \frac{\nu(K)}{\operatorname{Cap}_{\mathbf{I}_{\alpha p}, \frac{q}{q-p+1}}(K)} \asymp \sup _{K \in \mathcal{K}\left(\mathbb{R}^{N}\right)} \frac{\int_{K}\left(\mathbf{I}_{\alpha p}[\nu](y)\right)^{\frac{q}{p-1}} d y}{\operatorname{Cap}_{\mathbf{I}_{\alpha p}, \frac{q}{q-p+1}}(K)} \quad \text { for all } \nu \in \mathfrak{M}^{+}\left(\mathbb{R}^{N}\right) .
$$

From this we infer the equivalence between (a) and (b).

Step 2: Proof of (a) $\Leftrightarrow$ (c). By [21, Theorem 2.1] (a) is equivalent to

$$
\int_{\mathbb{R}^{N}}\left(\mathbf{I}_{\alpha p}\left[\chi_{B_{t}(x)} \mu\right](y)\right)^{\frac{q}{p-1}} d y \leq C \mu\left(B_{t}(x)\right),
$$

for any ball $B_{t}(x) \subset \mathbb{R}^{N}$. It is equivalent to (c) because of (2.5).

Step 3: By Proposition 2.4, we obtain (c) $\Rightarrow$ (d).

Step 4: Proof of $(\mathrm{d}) \Rightarrow(\mathrm{b})$. Set $d \nu(x)=\left(\mathbf{W}_{\alpha, p}[\mu](x)\right)^{q} d x$. Clearly, (d) implies

$$
\left(\mathbf{W}_{\alpha, p}[\nu](x)\right)^{q} d x \leq C d \nu(x) .
$$

Let $\mathbf{M}_{\nu}$ denote the centered Hardy-Littlewood maximal function defined for any $f \in L_{l o c}^{1}\left(\mathbb{R}^{N}, d \nu\right)$ by

$$
\mathbf{M}_{\nu} f(x)=\sup _{t>0} \frac{1}{\nu\left(B_{t}(x)\right)} \int_{B_{t}(x)}|f| d \nu .
$$


If $E \subset \mathbb{R}^{N}$ is a Borel set, we have

$$
\int_{\mathbb{R}^{N}}\left(\mathbf{W}_{\alpha, p}\left[\chi_{E} \nu\right]\right)^{q} d x \leq \int_{\mathbb{R}^{N}}\left(\mathbf{M}_{\nu} \chi_{E}\right)^{\frac{q}{p-1}}\left(\mathbf{W}_{\alpha, p}[\nu]\right)^{q} d x \leq C \int_{\mathbb{R}^{N}}\left(\mathbf{M}_{\nu} \chi_{E}\right)^{\frac{q}{p-1}} d \nu .
$$

Since $\mathbf{M}_{\nu}$ is bounded on $L^{s}\left(\mathbb{R}^{N}, d \nu\right), s>1$, by the Besicovitch's theorem, see e.g. [11], we deduce that

$$
\int_{\mathbb{R}^{N}}\left(\mathbf{W}_{\alpha, p}\left[\chi_{E} \nu\right]\right)^{q} d x \leq C \nu(E)
$$

for any Borel set $E$. Applying the equivalence of (a) and (c) with $\mu=\nu$, we derive (b).

The next result is the analogue of the previous one when the whole space is replaced by a ball. It can be proved in the same way, see also [23, Proof of Theorem 2.3].

Theorem 2.2 Let $1<p<N / \alpha, q>p-1, \omega \in \mathfrak{M}_{b}^{+}\left(B_{R}\left(x_{0}\right)\right)$ for some $R>0$ and $x_{0} \in \mathbb{R}^{N}$. Then, the following statements are equivalent:

(a) The inequality

$$
\omega(K) \leq C_{1} \operatorname{Cap}_{\mathbf{G}_{\alpha p}, \frac{q}{q-p+1}}(K),
$$

holds for any compact set $K \subset \mathbb{R}^{N}$, for some $C_{1}=C_{1}(R)>0$.

(b) The inequality

$$
\int_{K}\left(\mathbf{W}_{\alpha, p}^{4 R}[\omega](y)\right)^{q} d y \leq C_{2} \operatorname{Cap}_{\mathbf{G}_{\alpha p}, \frac{q}{q-p+1}}(K),
$$

holds for any compact set $K \subset \mathbb{R}^{N}$, for some $C_{2}=C_{2}(R)>0$.

(c) The inequality

$$
\int_{\mathbb{R}^{N}}\left(\mathbf{W}_{\alpha, p}^{4 R}\left[\chi_{B_{t}(x)} \omega\right](y)\right)^{q} d y \leq C_{3} \omega\left(B_{t}(x)\right)
$$

holds for any $x \in \mathbb{R}^{N}$ and $t>0$, for some $C_{3}=C_{3}(R)>0$.

(d) The inequality

$$
\mathbf{W}_{\alpha, p}^{4 R}\left[\left(\mathbf{W}_{\alpha, p}^{4 R}[\omega]\right)^{q}\right] \leq C_{4} \mathbf{W}_{\alpha, p}^{4 R}[\omega]
$$

holds almost everywhere in $B_{2 R}\left(x_{0}\right)$, for some $C_{4}=C_{4}(R)>0$.

The following stability result of the Lipschitz continuity of a measure with respect a capacity will be used several times in the sequel since we will approximate the initial data by smooth and truncated ones; its proof is easy, see e.g. [24, Lemma 2.7].

Proposition 2.3 Let $1<p<N / \alpha$ and $0<\beta<N / p, \mu \in \mathfrak{M}^{+}\left(\mathbb{R}^{N}\right), \omega \in \mathfrak{M}_{b}^{+}\left(B_{R}\left(x_{0}\right)\right)$ for some $R>0$ and $x_{0} \in \mathbb{R}^{N}$. Set $d \mu_{n}(x)=\left(\varphi_{n} * \mu\right)(x) d x, d \omega_{n}(x)=\left(\varphi_{n} * \omega\right)(x) d x$ where $\left\{\varphi_{n}\right\}$ is a sequence of mollifiers. Then,

(i) If inequality (2.1) in Theorem 2.1 holds with $q>\frac{(p-1) N}{N-\alpha p}$ and constant $C_{1}$, then

$$
\mu_{n}(K) \leq C C_{1} \operatorname{Cap}_{\mathbf{I}_{\alpha p}, \frac{q}{q-p+1}}(K) \text { for all } K \subset \mathbb{R}^{N}, n \in \mathbb{N}
$$

for some $C=C(N, \alpha, p, q)>0$ 
(ii) If inequality (2.6) in Theorem 2.2 holds with $q>p-1$ and constant $C_{2}$, then

$$
\omega_{n}(K) \leq C C_{2} \operatorname{Cap}_{\mathbf{G}_{\alpha p}, \frac{q}{q-p+1}}(K) \text { for all } K \subset \mathbb{R}^{N}, n \in \mathbb{N}
$$

for some $C=C(N, \alpha, p, q)>0$.

The next proposition is crucial as it gives pointwise estimates of interates of Wolff potentials of positive measures and connect them with the capacitary estimates of the Wolff potentials of the same measures.

Proposition 2.4 Let $1<p<N / \alpha$ and $0<\beta<N / p, \mu \in \mathfrak{M}^{+}\left(\mathbb{R}^{N}\right), \omega \in \mathfrak{M}_{b}^{+}\left(B_{R}\left(x_{0}\right)\right)$ for some $B_{R}\left(x_{0}\right) \subset \mathbb{R}^{N}$. Then,

(i) The inequality (2.3) in Theorem 2.1 with $q>\frac{(p-1) N}{N-\alpha p}$ implies that

$$
\mathbf{W}_{\beta, p}\left[\left(\mathbf{W}_{\alpha, p}[\mu]\right)^{q}\right] \leq C_{1} \mathbf{W}_{\beta, p}[\mu]<\infty,
$$

holds almost everywhere in $\mathbb{R}^{N}$, for some $C_{1}>0$.

(ii) The inequality (2.8) in Theorem 2.2 with $q>p-1$ implies that

$$
\mathbf{W}_{\beta, p}^{4 R}\left[\left(\mathbf{W}_{\alpha, p}^{4 R}[\omega]\right)^{q}\right] \leq C_{2} \mathbf{W}_{\beta, p}^{4 R}[\omega],
$$

holds almost everywhere in $B_{2 R}\left(x_{0}\right)$, for some $C_{2}>0$.

Proof. Assertion (i). First we assume that $\mu$ has compact support. Let $x \in \mathbb{R}^{N}$ and $t>0$. For any $y \in B_{t}(x)$,

$$
\begin{aligned}
\mathbf{W}_{\alpha, p}\left[\chi_{B_{t}(x)} \mu\right](y) & \geq \int_{2 t}^{+\infty}\left(\frac{\mu\left(B_{t}(x) \cap B_{r}(y)\right)}{r^{N-\alpha p}}\right)^{\frac{1}{p-1}} \frac{d r}{r} \\
& \geq \int_{2 t}^{+\infty}\left(\frac{\mu\left(B_{t}(x)\right)}{r^{N-\alpha p}}\right)^{\frac{1}{p-1}} \frac{d r}{r} \\
& \geq C\left(\frac{\mu\left(B_{t}(x)\right)}{t^{N-\alpha p}}\right)^{\frac{1}{p-1}} .
\end{aligned}
$$

From (2.3) we have

$$
\mu\left(B_{t}(x)\right) \geq C \int_{B_{t}(x)}\left(\mathbf{W}_{\alpha, p}\left[\chi_{B_{t}(x)} \mu\right](y)\right)^{q} d y \geq C t^{N}\left(\frac{\mu\left(B_{t}(x)\right)}{t^{N-\alpha p}}\right)^{\frac{1}{p-1}} .
$$

Hence, $\mu\left(B_{t}(x)\right) \leq C t^{N-\frac{\alpha p q}{q-p+1}}$. Therefore

$$
\int_{r}^{\infty}\left(\frac{\mu\left(B_{t}(x)\right)}{t^{N-\alpha p}}\right)^{\frac{1}{p-1}} \frac{d t}{t} \leq C r^{-\frac{\alpha p}{q-p+1}} .
$$


Since, $B_{t}(y) \subset B_{2 \max \{t, r\}}(x)$ for any $y \in B_{r}(x)$, we have

$$
\begin{aligned}
& \int_{B_{r}(x)}\left(\mathbf{W}_{\alpha, p}[\mu](y)\right)^{q} d y \leq C \int_{B_{r}(x)}\left(\int_{0}^{r}\left(\frac{\mu\left(B_{t}(y) \cap B_{2 r}(x)\right)}{t^{N-\alpha p}}\right)^{\frac{1}{p-1}} \frac{d t}{t}\right)^{q} d y \\
&+C \int_{B_{r}(x)}\left(\int_{r}^{\infty}\left(\frac{\mu\left(B_{t}(y) \cap B_{2 t}(x)\right)}{t^{N-\alpha p}}\right)^{\frac{1}{p-1}} \frac{d t}{t}\right)^{q} d y \\
& \leq C \int_{B_{r}(x)}\left(\mathbf{W}_{\alpha, p}\left[\chi_{B_{2 r}(x)} \mu\right]\right)^{q} d y+C r^{N}\left(\int_{r}^{\infty}\left(\frac{\mu\left(B_{2 t}(x)\right)}{t^{N-\alpha p}}\right)^{\frac{1}{p-1}} \frac{d t}{t}\right)^{q} \\
& \leq C \mu\left(B_{2 r}(x)\right)+C r^{N}\left(\int_{r}^{\infty}\left(\frac{\mu\left(B_{2 t}(x)\right)}{t^{N-\alpha p}}\right)^{\frac{1}{p-1}} \frac{d t}{t}\right)^{q} .
\end{aligned}
$$

Note that, in the last inequality, we have used (2.3). Thus,

$$
\begin{aligned}
& \mathbf{W}_{\beta, p}\left[\left(\mathbf{W}_{\alpha, p}[\mu]\right)^{q}\right](x)=\int_{0}^{\infty}\left(\frac{\int_{B_{r}(x)}\left(\mathbf{W}_{\alpha, p}[\mu](y)\right)^{q} d y}{r^{N-\beta p}}\right)^{\frac{1}{p-1}} \frac{d r}{r} \\
& \quad \leq C \int_{0}^{\infty}\left(\frac{\mu\left(B_{2 r}(x)\right)}{r^{N-\beta p}}\right)^{\frac{1}{p-1}} \frac{d r}{r}+C \int_{0}^{\infty} r^{\frac{\beta p}{p-1}-1}\left(\int_{r}^{\infty}\left(\frac{\mu\left(B_{2 t}(x)\right)}{t^{N-\alpha p}}\right)^{\frac{1}{p-1}} \frac{d t}{t}\right)^{\frac{q}{p-1}} d r .
\end{aligned}
$$

Therefore, it remains to prove

$$
\int_{0}^{\infty} r^{\frac{\beta p}{p-1}-1}\left(\int_{r}^{\infty}\left(\frac{\mu\left(B_{2 t}(x)\right)}{t^{N-\alpha p}}\right)^{\frac{1}{p-1}} \frac{d t}{t}\right)^{\frac{q}{p-1}} d r \leq C \mathbf{W}_{\beta, p}[\mu](x) .
$$

Notice that

$$
r^{\frac{\beta p}{p-1}}\left(\int_{r}^{\infty}\left(\frac{\mu\left(B_{2 t}(x)\right)}{t^{N-\alpha p}}\right)^{\frac{1}{p-1}} \frac{d t}{t}\right)^{\frac{q}{p-1}} \rightarrow 0 \text { as } t \rightarrow 0
$$

and

$$
r^{\frac{\beta p}{p-1}}\left(\int_{r}^{\infty}\left(\frac{\mu\left(B_{2 t}(x)\right)}{t^{N-\alpha p}}\right)^{\frac{1}{p-1}} \frac{d t}{t}\right)^{\frac{q}{p-1}} \leq C r^{\frac{\beta p}{p-1}-\frac{N-\alpha p}{p-1} \frac{q}{p-1}}\left(\mu\left(\mathbb{R}^{N}\right)\right)^{\frac{q}{(p-1)^{2}}} \rightarrow 0
$$

as $t \rightarrow \infty$, since $\frac{\beta p}{p-1}-\frac{N-\alpha p}{p-1} \frac{q}{p-1}<\frac{\beta p}{p-1}-\frac{N}{p-1}<0$. Hence, using integration by parts and inequality (2.14), we have

$$
\begin{aligned}
& \int_{0}^{\infty} r^{\frac{\beta p}{p-1}-1}\left(\int_{r}^{\infty}\left(\frac{\mu\left(B_{2 t}(x)\right)}{t^{N-\alpha p}}\right)^{\frac{1}{p-1}} \frac{d t}{t}\right)^{\frac{q}{p-1}} d r \\
& =\frac{q}{\beta p} \int_{0}^{\infty} r^{\frac{\beta p}{p-1}}\left(\int_{r}^{\infty}\left(\frac{\mu\left(B_{2 t}(x)\right)}{t^{N-\alpha p}}\right)^{\frac{1}{p-1}} \frac{d t}{t}\right)^{\frac{q}{p-1}-1}\left(\frac{\mu\left(B_{2 r}(x)\right)}{r^{N-\alpha p}}\right)^{\frac{1}{p-1}} \frac{d r}{r} \\
& \leq C \int_{0}^{\infty} r^{\frac{\beta p}{p-1}}\left(r^{-\frac{\alpha p}{q-p+1}}\right)^{\frac{q}{p-1}-1}\left(\frac{\mu\left(B_{2 r}(x)\right)}{r^{N-\alpha p}}\right)^{\frac{1}{p-1}} \frac{d r}{r} \\
& =C \mathbf{W}_{\beta, p}[\mu](x) .
\end{aligned}
$$


Next, we assume that $\mu$ is not necessarily compactly supported. From the previous step,

$$
\mathbf{W}_{\beta, p}\left[\left(\mathbf{W}_{\alpha, p}\left[\chi_{B_{n}(0)} \mu\right]\right)^{q}\right] \leq C \mathbf{W}_{\beta, p}\left[\chi_{B_{n}(0)} \mu\right] \leq C \mathbf{W}_{\beta, p}[\mu]<\infty \text { a.e in } \mathbb{R}^{N} .
$$

Then we derive (2.12) by Fatou's lemma.

Assertion (ii). For any $x \in B_{2 R}\left(x_{0}\right), 0<t<R / 2$ and $y \in B_{t}(x)$,

$$
\begin{aligned}
\mathbf{W}_{\alpha, p}^{4 R}\left[\chi_{B_{t}(x)} \omega\right](y) & \geq \int_{2 t}^{4 R}\left(\frac{\omega\left(B_{t}(x) \cap B_{r}(y)\right)}{r^{N-\alpha p}}\right)^{\frac{1}{p-1}} \frac{d r}{r} \\
& \geq C\left(\frac{\omega\left(B_{t}(x)\right)}{t^{N-\alpha p}}\right)^{\frac{1}{p-1}} .
\end{aligned}
$$

From (2.8) we have

$$
\omega\left(B_{t}(x)\right) \geq C \int_{B_{t}(x)}\left(\mathbf{W}_{\alpha, p}^{4 R}\left[\chi_{B_{t}(x)} \omega\right](y)\right)^{q} d y \geq C t^{N}\left(\frac{\omega\left(B_{t}(x)\right)}{t^{N-\alpha p}}\right)^{\frac{1}{p-1}} .
$$

Hence, $\omega\left(B_{t}(x)\right) \leq C t^{N-\frac{\alpha p q}{q-p+1}}$ for all $t \in(0, R / 2)$ and $x \in B_{2 R}\left(x_{0}\right)$. It implies

$$
\int_{r}^{4 R}\left(\frac{\omega\left(B_{2 t}(x)\right)}{t^{N-\alpha p}}\right)^{\frac{1}{p-1}} \frac{d t}{t} \leq C r^{-\frac{\alpha p}{q-p+1}} \text { for all } x \in B_{2 R}\left(x_{0}\right), 0<r<4 R .
$$

Since $B_{t}(y) \subset B_{2 \max \{t, r\}}(x)$ for any $0<r<4 R$ and $y \in B_{r}(x)$,

$$
\begin{aligned}
& \int_{B_{r}(x)}\left(\mathbf{W}_{\alpha, p}^{4 R}[\omega](y)\right)^{q} d y \leq C \int_{B_{r}(x)}\left(\int_{0}^{r}\left(\frac{\omega\left(B_{t}(y) \cap B_{2 r}(x)\right)}{t^{N-\alpha p}}\right)^{\frac{1}{p-1}} \frac{d t}{t}\right)^{q} d y \\
&+C \int_{B_{r}(x)}\left(\int_{r}^{4 R}\left(\frac{\omega\left(B_{t}(y) \cap B_{2 t}(x)\right)}{t^{N-\alpha p}}\right)^{\frac{1}{p-1}} \frac{d t}{t}\right)^{q} d y \\
& \leq C \int_{B_{r}(x)}\left(\mathbf{W}_{\alpha, p}^{4 R}\left[\chi_{B_{2 r}(x)} \omega\right]\right)^{q} d y+C r^{N}\left(\int_{r}^{4 R}\left(\frac{\mu\left(B_{2 t}(x)\right)}{t^{N-\alpha p}}\right)^{\frac{1}{p-1}} \frac{d t}{t}\right)^{q} \\
& \leq C \mu\left(B_{2 r}(x)\right)+C r^{N}\left(\int_{r}^{4 R}\left(\frac{\mu\left(B_{2 t}(x)\right)}{t^{N-\alpha p}}\right)^{\frac{1}{p-1}} \frac{d t}{t}\right)^{q} .
\end{aligned}
$$

In the last inequality we have used (2.8). Thus, as above, we only need to prove that

$$
\int_{0}^{4 R} r^{\frac{\beta p}{p-1}-1}\left(\int_{r}^{4 R}\left(\frac{\omega\left(B_{2 t}(x)\right)}{t^{N-\alpha p}}\right)^{\frac{1}{p-1}} \frac{d t}{t}\right)^{\frac{q}{p-1}} d r \leq C \mathbf{W}_{\beta, p}^{2 R}[\omega](x) .
$$

Using integration by parts and (2.15)

$$
\begin{aligned}
& \int_{0}^{4 R} r^{\frac{\beta p}{p-1}-1}\left(\int_{r}^{4 R}\left(\frac{\omega\left(B_{2 t}(x)\right)}{t^{N-\alpha p}}\right)^{\frac{1}{p-1}} \frac{d t}{t}\right)^{\frac{q}{p-1}} d r \\
& =\frac{q}{\beta p} \int_{0}^{4 R} r^{\frac{\beta p}{p-1}}\left(\int_{r}^{4 R}\left(\frac{\omega\left(B_{2 t}(x)\right)}{t^{N-\alpha p}}\right)^{\frac{1}{p-1}} \frac{d t}{t}\right)^{\frac{q}{p-1}-1}\left(\frac{\omega\left(B_{2 r}(x)\right)}{r^{N-\alpha p}}\right)^{\frac{1}{p-1}} \frac{d r}{r} \\
& \leq C \int_{0}^{4 R} r^{\frac{\beta p}{p-1}}\left(r^{-\frac{\alpha p}{q-p+1}}\right)^{\frac{q}{p-1}-1}\left(\frac{\omega\left(B_{2 r}(x)\right)}{r^{N-\alpha p}}\right)^{\frac{1}{p-1}} \frac{d r}{r} \\
& \leq C \mathbf{W}_{\beta, p}^{8 R}[\omega](x) \leq C \mathbf{W}_{\beta, p}^{4 R}[\omega](x),
\end{aligned}
$$


since $\mathbf{W}_{\beta, p}^{8 R}[\omega](x) \leq C \mathbf{W}_{\beta, p}^{4 R}[\omega](x)$ for any $x \in B_{2 R}\left(x_{0}\right)$, because $\operatorname{supp} \omega \subset B_{2 R}\left(x_{0}\right)$.

The next result is at the core of our construction since it connects the integral of product of Wolff potentials to some power to the integral of a new Wolff potential. In this highly technical construction, the role of Hardy Littlewood maximal function plays an important role as well as classical tools from harmonic analysis such as the Vitali Covering Lemma.

Theorem 2.5 Let $\alpha, \beta, q_{1}, q_{2}>0, \alpha>\beta, 1<p<\frac{N}{\alpha}, q_{1}+q_{2}>p-1, q_{2}<\frac{N(p-1)}{N-\beta p}$ and $\frac{\alpha p q_{1}+\beta p q_{2}}{q_{1}+q_{2}}<N$. Then, there holds

$$
\begin{aligned}
\int_{\mathbb{R}^{N}}\left(\mathbf{M}_{\frac{\alpha p q_{1}+\beta p q_{2}}{q_{1}+q_{2}}}[\mu](x)\right)^{\frac{q_{1}+q_{2}}{p-1}} d x & \asymp \int_{\mathbb{R}^{N}}\left(\mathbf{W}_{\frac{\alpha p q_{1}+\beta p q_{2}}{q_{1}+q_{2}}, p}[\mu](x)\right)^{q_{1}+q_{2}} d x \\
& \asymp \int_{\mathbb{R}^{N}}\left(\mathbf{W}_{\alpha, p}[\mu](x)\right)^{q_{1}}\left(\mathbf{W}_{\beta, p}[\mu](x)\right)^{q_{2}} d x,
\end{aligned}
$$

for any $\mu \in \mathfrak{M}^{+}\left(\mathbb{R}^{N}\right)$, and

$$
\begin{aligned}
\int_{\mathbb{R}^{N}}\left(\mathbf{M}_{\frac{\alpha p q+\beta p q_{2}}{q_{1}+q_{2}}}^{2 R}[\omega](x)\right)^{\frac{q_{1}+q_{2}}{p-1}} & \asymp \int_{\mathbb{R}^{N}}\left(\mathbf{W}_{\frac{\alpha p q_{1}+\beta p q_{2}}{q_{1}+q_{2}}, p}^{2 R}[\omega](x)\right)^{q_{1}+q_{2}} d x \\
& \asymp \int_{\mathbb{R}^{N}}\left(\mathbf{W}_{\alpha, p}^{2 R}[\omega](x)\right)^{q_{1}}\left(\mathbf{W}_{\beta, p}^{2 R}[\omega](x)\right)^{q_{2}} d x,
\end{aligned}
$$

for any $R>0$ and $\omega \in \mathfrak{M}^{+}\left(\mathbb{R}^{N}\right)$ with diam $(\operatorname{supp} \omega) \leq R$.

For proving this theorem we need several intermediate results. For any $\alpha \in(0, N), s>0$, $R \in(0, \infty]$ we denote

$$
\mathbf{L}_{\alpha, s}^{R}[\mu](x)=\int_{0}^{R}\left(\frac{\mu\left(B_{t}(x)\right)}{t^{N-\alpha}}\right)^{s} \frac{d t}{t},
$$

and $\mathbf{L}_{\alpha, s}[\mu]:=\mathbf{L}_{\alpha, s}^{\infty}[\mu]$ when $R=\infty$. We notice that $\mathbf{L}_{\alpha, s}^{R}$ is actually a Wolff potential since

$$
\mathbf{L}_{\alpha, s}^{R}[\mu]=\mathbf{W}_{\frac{\alpha s}{s+1}, \frac{s+1}{s}}^{R}[\mu] \quad \text { and } \quad \mathbf{L}_{\alpha, s}[\mu]=\mathbf{W}_{\frac{\alpha s}{s+1}, \frac{s+1}{s}}[\mu] .
$$

Lemma 2.6 Let $\alpha_{1}, \alpha_{2}, s_{1}, s_{2}>0,0<\alpha_{2}<\alpha_{1}<N$. There exist $C=C\left(N, \alpha_{1}, \alpha_{2}, s_{1}, s_{2}\right)>$ 0 and $\varepsilon_{0}=\varepsilon_{0}\left(N, \alpha_{1}, \alpha_{2}, s_{1}, s_{2}\right)>0$ such that for any $\mu \in \mathfrak{M}_{+}\left(\mathbb{R}^{N}\right), R \in(0, \infty], \varepsilon \in\left(0, \varepsilon_{0}\right)$ and $\lambda>0$, the inequality

$$
\left|\left\{\mathbf{L}_{\alpha_{1}, s_{1}}^{2 R}[\mu] \mathbf{L}_{\alpha_{2}, s_{2}}^{2 R}[\mu]>\varepsilon^{1 / 2} \lambda\right\}\right|<\infty,
$$

implies

$$
\begin{aligned}
\mid\left\{\mathbf{L}_{\alpha_{1}, s_{1}}^{R}[\mu] \mathbf{L}_{\alpha_{2}, s_{2}}^{R}[\mu]>a \lambda\right\} \cap\{ & \left.\left(\mathbf{M}_{\frac{\alpha_{1} s_{1}+\alpha_{2} s_{2}}{s_{1}+s_{2}}}^{2 R}[\mu]\right)^{s_{1}+s_{2}} \leq \varepsilon \lambda\right\} \mid \\
& \leq C \varepsilon^{\frac{\varepsilon_{2}\left(N-\alpha_{2}\right)}{2 s_{2}\left(N-\alpha_{1}\right.}}\left|\left\{\mathbf{L}_{\alpha_{1}, s_{1}}^{2 R}[\mu] \mathbf{L}_{\alpha_{2}, s_{2}}^{2 R}[\mu]>\varepsilon^{1 / 2} \lambda\right\}\right| .
\end{aligned}
$$

To prove this, we need the following two lemmas:

Lemma 2.7 Let $0<\alpha<N$ and $s>0$. There exists $C=C(N, \alpha, s)$ such that

$$
\left|\left\{\mathbf{L}_{\alpha, s}[\omega]>\lambda\right\}\right| \leq C\left(\frac{\left(\omega\left(\mathbb{R}^{N}\right)\right)^{s}}{\lambda}\right)^{\frac{N}{s(N-\alpha)}} \quad \text { for all } \lambda>0,
$$

for any $\omega \in \mathfrak{M}_{b}^{+}\left(\mathbb{R}^{N}\right)$. 
Proof. It is easy to see that $\mathbf{L}_{\alpha, s}[\omega](x) \leq C(\mathbf{M}(\omega)(x))^{\frac{s(N-\alpha)}{N}}\left(\omega\left(\mathbb{R}^{N}\right)\right)^{\frac{\alpha s}{N}}$. Thus, thanks to boundedness of the operator $\mathbf{M}$ from $\mathfrak{M}_{b}^{+}\left(\mathbb{R}^{N}\right)$ to $L^{1, \infty}\left(\mathbb{R}^{d}\right)$, we get $(2.22)$. The proof is complete.

The next result is a consequence of Vitali Covering Lemma.

Lemma 2.8 Let $0<\varepsilon<1, R>0$ and $B:=B_{R}\left(x_{0}\right)$ for some $x_{0} \in \mathbb{R}^{N}$. Let $E \subset F \subset B$ be two measurable sets in $\mathbb{R}^{N}$ with $|E|<\varepsilon|B|$ and satisfying the following property: for all $x \in B$ and $r \in(0, R]$, we have $B_{r}(x) \cap B \subset F$ provided $\left|E \cap B_{r}(x)\right| \geq \varepsilon\left|B_{r}(x)\right|$. Then $|E| \leq C \varepsilon|F|$ for some $C=C(N)$.

Proof of Lemma 2.6. We only consider the case $R<\infty$, the case $R=\infty$ being similar. Let $\left\{B_{R}\left(x_{j}\right)\right\}$ be a cover of $\mathbb{R}^{N}$ such that, for some constant $M=M(N)>0$,

$$
\sum_{j} \chi_{B_{R / 4}\left(x_{j}\right)}(x) \leq M \quad \text { for all } x \in \mathbb{R}^{N} .
$$

It is sufficient to show that there exist constants $c_{1}, c_{2}>0$ and $\varepsilon_{0} \in(0,1)$ depending on $N, \alpha_{1}, \alpha_{2}, s_{1}, s_{2}, p$ such that for any $B \in\left\{B_{R / 4}\left(x_{j}\right)\right\}, \lambda>0$ and $\varepsilon \in\left(0, \varepsilon_{0}\right)$, there holds

$$
\begin{aligned}
\mid B \cap\left\{\mathbf{L}_{\alpha_{1}, s_{1}}^{R}[\mu] \mathbf{L}_{\alpha_{1}, s_{1}}^{R}[\mu]>\right. & a \lambda\} \cap\left\{\left(\mathbf{M}_{\frac{\alpha_{1} s_{1}+\alpha_{2} s_{2}}{s_{1}+s_{2}}}^{2 R}[\mu]\right)^{s_{1}+s_{2}} \leq \varepsilon \lambda\right\} \mid \\
& \leq C \varepsilon^{\frac{N}{s_{2}\left(N-\alpha_{2}\right)}}\left|B \cap\left\{\mathbf{L}_{\alpha_{1}, s_{1}}^{2 R}[\mu] \mathbf{L}_{\alpha_{1}, s_{1}}^{2 R}[\mu]>\varepsilon^{1 / 2} \lambda\right\}\right|,
\end{aligned}
$$

where

$$
a=1+\left(\frac{s_{1}+s_{2}}{s_{1} s_{2}\left(\alpha_{1}-\alpha_{2}\right)}\right)^{2}+2^{\left(N-\alpha_{1}\right) s_{1}+\left(N-\alpha_{2}\right) s_{2}+1} .
$$

Fix $\lambda>0$ and $0<\varepsilon<\min \left\{1 / 10,2^{-\frac{10 s_{1} s_{2}\left(\alpha_{1}-\alpha_{2}\right)}{s_{1}+s_{2}}}\right\}$. We set

$$
E=B \cap\left\{\mathbf{L}_{\alpha_{1}, s_{1}}^{R}[\mu] \mathbf{L}_{\alpha_{1}, s_{1}}^{R}[\mu]>a \lambda\right\} \cap\left\{\left(\mathbf{M}_{\frac{\alpha_{1} s_{1}+\alpha_{2} s_{2}}{s_{1}+s_{2}}}^{2 R}[\mu]\right)^{s_{1}+s_{2}} \leq \varepsilon \lambda\right\},
$$

and

$$
F=B \cap\left\{\mathbf{L}_{\alpha_{1}, s_{1}}^{2 R}[\mu] \mathbf{L}_{\alpha_{1}, s_{1}}^{2 R}[\mu]>\varepsilon^{1 / 2} \lambda\right\} .
$$

Thanks to Lemma 2.8 we will obtain (2.23) provided we verify the following two claims:

$$
|E| \leq C \varepsilon^{\frac{N}{2 s_{2}\left(N-\alpha_{2}\right)}}|B|,
$$

and, for any $x \in B$ and $0<r \leq R / 4$,

$$
\left|E \cap B_{r}(x)\right|<C \varepsilon^{\frac{N}{2 s_{2}\left(N-\alpha_{2}\right)}}\left|B_{r}(x)\right|,
$$

whenever $B_{r}(x) \cap B \cap F^{c} \neq \emptyset$ and $E \cap B_{r}(x) \neq \emptyset$. 
Proof of (2.24): For any $x \in E$, we have

$$
\begin{aligned}
\mathbf{L}_{\alpha_{1}, s_{1}}^{R}[\mu](x) & \leq \int_{0}^{R}\left(t^{-\frac{\alpha_{1} s_{1}+\alpha_{2} s_{2}}{s_{1}+s_{2}}+\alpha_{1}} \mathbf{M}_{\frac{\alpha_{1} s_{1}+\alpha_{2} s_{2}}{s_{1}+s_{2}}}^{2 R}[\mu](x)\right)^{s_{1}} \frac{d t}{t} \\
& \leq \frac{s_{1}+s_{2}}{s_{1} s_{2}\left(\alpha_{1}-\alpha_{2}\right)} R^{\frac{s_{1} s_{2}\left(\alpha_{1}-\alpha_{2}\right)}{s_{1}+s_{2}}}(\varepsilon \lambda)^{\frac{s_{1}}{s_{1}+s_{2}}}
\end{aligned}
$$

Hence, the inequality $\mathbf{L}_{\alpha_{1}, s_{1}}^{R}[\mu](x) \mathbf{L}_{\alpha_{2}, s_{2}}^{R}[\mu](x)>\lambda$ implies

$$
\mathbf{L}_{\alpha_{2}, s_{2}}^{R}[\mu](x)>\frac{s_{1} s_{2}\left(\alpha_{1}-\alpha_{2}\right)}{s_{1}+s_{2}} R^{-\frac{s_{1} s_{2}\left(\alpha_{1}-\alpha_{2}\right)}{s_{1}+s_{2}}} \varepsilon^{-\frac{s_{1}}{s_{1}+s_{2}}} \lambda^{\frac{s_{2}}{s_{1}+s_{2}}}
$$

Clearly, $\mathbf{L}_{\alpha_{2}, s_{2}}^{R}[\mu]=\mathbf{L}_{\alpha_{2}, s_{2}}^{R}\left[\chi_{B_{2} R}\left(y_{0}\right) \mu\right]$ in $B$ for any $y_{0} \in B$. Fix $y_{0} \in E$, we have

$$
|E| \leq\left|\left\{\mathbf{L}_{\alpha_{2}, s_{2}}\left[\chi_{B_{2 R}\left(y_{0}\right)} \mu\right]>\frac{s_{1} s_{2}\left(\alpha_{1}-\alpha_{2}\right)}{s_{1}+s_{2}} R^{-\frac{s_{1} s_{2}\left(\alpha_{1}-\alpha_{2}\right)}{s_{1}+s_{2}}} \varepsilon^{-\frac{s_{1}}{s_{1}+s_{2}}} \lambda^{\frac{s_{2}}{s_{1}+s_{2}}}\right\}\right| .
$$

Using (2.21) from Lemma 2.7 and the fact that $\left(\mathbf{M}_{\frac{\alpha_{1} s_{1}+\alpha_{2} s_{2}}{s_{1}+s_{2}}}^{2 R}[\mu]\left(y_{0}\right)\right)^{s_{1}+s_{2}} \leq \varepsilon \lambda$, we get

$$
\begin{aligned}
|E| & \leq C\left(\frac{\left(\mu\left(B_{2 R}\left(y_{0}\right)\right)\right)^{s_{2}}}{R^{-\frac{s_{1} s_{2}\left(\alpha_{1}-\alpha_{2}\right)}{s_{1}+s_{2}}} \varepsilon^{-\frac{s_{1}}{s_{1}+s_{2}} \lambda^{\frac{s_{2}}{s_{1}+s_{2}}}}}\right)^{\frac{N}{s_{2}\left(N-\alpha_{2}\right)}} \\
& \leq C\left(\frac{(\varepsilon \lambda)^{\frac{s_{2}}{s_{1}+s_{2}}}(2 R)^{s_{2} N-s_{2} \frac{\alpha_{1} s_{1}+\alpha_{2} s_{2}}{s_{1}+s_{2}}}}{R^{-\frac{s_{1} s_{2}\left(\alpha_{1}-\alpha_{2}\right)}{s_{1}+s_{2}}} \varepsilon^{-\frac{s_{1}}{s_{1}+s_{2}} \lambda^{\frac{s_{2}}{s_{1}+s_{2}}}}}\right)^{\frac{N}{s_{2}\left(N-\alpha_{2}\right)}} \\
& =C \varepsilon^{\frac{N}{s_{2}\left(N-\alpha_{2}\right)}}|B| \\
& \leq C \varepsilon^{\frac{N}{s_{2}\left(N-\alpha_{2}\right)}}|B| .
\end{aligned}
$$

We obtain (2.24).

Proof of (2.25): Take $x \in B$ and $0<r \leq R / 4$. Now assume that $B_{r}(x) \cap B \cap F^{c} \neq \emptyset$ and $E \cap B_{r}(x) \neq \emptyset$, then there exists $x_{1} \in B_{r}(x) \cap B$ such that $\mathbf{L}_{\alpha_{1}, s_{1}}^{2 R}[\mu]\left(x_{1}\right) \mathbf{L}_{\alpha_{2}, s_{2}}^{2 R}[\mu]\left(x_{1}\right) \leq \varepsilon^{1 / 2} \lambda$. We need to prove that

$$
\left|E \cap B_{r}(x)\right|<C \varepsilon^{\frac{N}{2 s_{2}\left(N-\alpha_{2}\right)}}\left|B_{r}(x)\right| .
$$

To do this, we can write

$$
\mathbf{L}_{\alpha_{1}, s_{1}}^{R}[\mu](y) \mathbf{L}_{\alpha_{1}, s_{1}}^{R}[\mu](y)=\mathbf{T}_{1}(y)+\mathbf{T}_{2}(y)+\mathbf{T}_{3}(y)+\mathbf{T}_{4}(y)
$$

where

$$
\begin{aligned}
& \mathbf{T}_{1}(y)=\mathbf{L}_{\alpha_{1}, s_{1}}^{4 r}[\mu](y) \mathbf{L}_{\alpha_{2}, s_{2}}^{4 r}[\mu](y), \\
& \mathbf{T}_{2}(y)=\mathbf{L}_{\alpha_{1}, s_{1}}^{4 r}[\mu](y) \int_{4 r}^{R}\left(\frac{\mu\left(B_{\rho}(y)\right)}{\rho^{N-\alpha_{2}}}\right)^{s_{2}} \frac{d \rho}{\rho}, \\
& \mathbf{T}_{3}(y)=\int_{4 r}^{R}\left(\frac{\mu\left(B_{\rho}(y)\right)}{\rho^{N-\alpha_{1}}}\right)^{s_{1}} \frac{d \rho}{\rho} \mathbf{L}_{\alpha_{2}, s_{2}}^{4 r}[\mu](y), \\
& \mathbf{T}_{4}(y)=\int_{4 r}^{R}\left(\frac{\mu\left(B_{\rho}(y)\right)}{\rho^{N-\alpha_{1}}}\right)^{s_{1}} \frac{d \rho}{\rho} \int_{4 r}^{R}\left(\frac{\mu\left(B_{\rho}(y)\right)}{\rho^{N-\alpha_{2}}}\right)^{s_{2}} \frac{d \rho}{\rho} .
\end{aligned}
$$


For all $y \in E \cap B_{r}(x)$, we have

$$
\begin{aligned}
\mathbf{T}_{2}(y) & \leq \int_{0}^{4 r}\left(\rho^{-\frac{\alpha_{1} s_{1}+\alpha_{2} s_{2}}{s_{1}+s_{2}}+\alpha_{1}} \mathbf{M}_{\frac{\alpha_{1} s_{1}+\alpha_{2} s_{2}}{s_{1}+s_{2}}}^{R}[\mu](y)\right)^{s_{1}} \frac{d \rho}{\rho} \\
& \times \int_{4 r}^{R}\left(\rho^{-\frac{\alpha_{1} s_{1}+\alpha_{2} s_{2}}{s_{1}+s_{2}}+\alpha_{2}} \mathbf{M}_{\frac{\alpha_{1} s_{1}+\alpha_{2} s_{2}}{s_{1}+s_{2}}}^{R}[\mu](y)\right)^{s_{2}} \frac{d \rho}{\rho} \\
& \leq\left(\frac{s_{1}+s_{2}}{s_{1} s_{2}\left(\alpha_{1}-\alpha_{2}\right)}\right)^{2} r^{\frac{\left(\alpha_{1}-\alpha_{2}\right) s_{1} s_{2}}{s_{1}+s_{2}}} r^{-\frac{\left(\alpha_{1}-\alpha_{2}\right) s_{1} s_{2}}{s_{1}+s_{2}}}\left(\mathbf{M}_{\frac{\alpha_{1} s_{1}+\alpha_{2} s_{2}}{s_{1}+s_{2}}}^{R}[\mu](y)\right)^{s_{1}+s_{2}} \\
& \leq\left(\frac{s_{1}+s_{2}}{s_{1} s_{2}\left(\alpha_{1}-\alpha_{2}\right)}\right)^{2} \varepsilon \lambda,
\end{aligned}
$$

also

$$
\begin{aligned}
\mathbf{T}_{4}(y) & \leq \int_{4 r}^{R}\left(\frac{\mu\left(B_{2 \rho}\left(x_{1}\right)\right)}{\rho^{N-\alpha_{1}}}\right)^{s_{1}} \frac{d \rho}{\rho} \int_{4 r}^{R}\left(\frac{\mu\left(B_{2 \rho}\left(x_{1}\right)\right)}{\rho^{N-\alpha_{2}}}\right)^{s_{2}} \frac{d \rho}{\rho} \\
& \leq 2^{\left(N-\alpha_{1}\right) s_{1}+\left(N-\alpha_{2}\right) s_{2}} \mathbf{L}_{\alpha_{1}, s_{1}}^{2 R}[\mu]\left(x_{1}\right) \mathbf{L}_{\alpha_{2}, s_{2}}^{2 R}[\mu]\left(x_{1}\right) \\
& \leq 2^{\left(N-\alpha_{1}\right) s_{1}+\left(N-\alpha_{2}\right) s_{2}} \lambda,
\end{aligned}
$$

and

$$
\begin{aligned}
\mathbf{T}_{3}(y) & \leq \int_{4 r}^{R}\left(\frac{\mu\left(B_{2 \rho}\left(x_{1}\right)\right)}{\rho^{N-\alpha_{1}}}\right)^{s_{1}} \frac{d \rho}{\rho} \mathbf{L}_{\alpha_{2}, s_{2}}^{4 r}[\mu](y) \\
& \leq 2^{s_{1}\left(N-\alpha_{1}\right)} \mathbf{L}_{\alpha_{1}, s_{1}}^{2 R}[\mu]\left(x_{1}\right) \mathbf{L}_{\alpha_{2}, s_{2}}^{4 r}[\mu](y),
\end{aligned}
$$

Thus,

$$
\left|E \cap B_{r}(x)\right| \leq \mathbf{Y}_{1}+\mathbf{Y}_{2}+\mathbf{Y}_{3}+\mathbf{Y}_{4}
$$

where

$$
\begin{aligned}
& \mathbf{Y}_{1}=\left|E \cap B_{r}(x) \cap\left\{\mathbf{T}_{1}>\lambda\right\} \cap\left\{\left(\mathbf{M}_{\frac{\alpha_{1} s_{1}+\alpha_{2} s_{2}}{s_{1}+s_{2}}}^{2 R}[\mu]\right)^{s_{1}+s_{2}} \leq \varepsilon \lambda\right\}\right|, \\
& \mathbf{Y}_{2}=\left|E \cap B_{r}(x) \cap\left\{\mathbf{T}_{2}>\left(\frac{s_{1}+s_{2}}{s_{1} s_{2}\left(\alpha_{1}-\alpha_{2}\right)}\right)^{2} \lambda\right\} \cap\left\{\left(\mathbf{M}_{\frac{\alpha_{1} s_{1}+\alpha_{2} s_{2}}{s_{1}+s_{2}}}^{2 R}[\mu]\right)^{s_{1}+s_{2}} \leq \varepsilon \lambda\right\}\right|, \\
& \mathbf{Y}_{3}=\left|E \cap B_{r}(x) \cap\left\{\mathbf{T}_{3}>2^{s_{1}\left(N-\alpha_{1}\right)} \lambda\right\} \cap\left\{\left(\mathbf{M}_{\frac{\alpha_{1} s_{1}+\alpha_{2} s_{2}}{s_{1}+s_{2}}}^{2 R}[\mu]\right)^{s_{1}+s_{2}} \leq \varepsilon \lambda\right\}\right|, \\
& \mathbf{Y}_{4}=\left|E \cap B_{r}(x) \cap\left\{\mathbf{T}_{4}>2^{\left(N-\alpha_{1}\right) s_{1}+\left(N-\alpha_{2}\right) s_{2}} \lambda\right\} \cap\left\{\left(\mathbf{M}_{\frac{\alpha_{1} s_{1}+\alpha_{2} s_{2}}{s_{1}+s_{2}}}^{2 R}[\mu]\right)^{s_{1}+s_{2}} \leq \varepsilon \lambda\right\}\right| .
\end{aligned}
$$

As in the proof of (2.24), it can be shown that

$$
\mathbf{Y}_{1} \leq c_{11} \varepsilon^{\frac{N}{2 s_{2}\left(N-\alpha_{2}\right)}}\left|B_{r}(x)\right| .
$$

From (2.27)-(2.29), we obtain $\mathbf{Y}_{2}=\mathbf{Y}_{4}=0$ and

$$
\begin{aligned}
\mathbf{Y}_{3} & \leq\left|B_{r}(x) \cap\left\{\mathbf{L}_{\alpha_{2}, s_{2}}^{4 r}[\mu]>\lambda\left(\mathbf{L}_{\alpha_{1}, s_{1}}^{2 R}[\mu]\left(x_{1}\right)\right)^{-1}\right\}\right| \\
& =\left|B_{r}(x) \cap\left\{\mathbf{L}_{\alpha_{2}, s_{2}}^{4 r}\left[\chi_{B_{6 r}\left(x_{1}\right)} \mu\right]>\lambda\left(\mathbf{L}_{\alpha_{1}, s_{1}}^{2 R}[\mu]\left(x_{1}\right)\right)^{-1}\right\}\right|
\end{aligned}
$$


since $B_{4 r}(y) \subset B_{6 r}\left(x_{1}\right)$ for all $y \in B_{r}(x)$. Using (2.21) from Lemma 2.7, we get

$$
\begin{aligned}
\mathbf{Y}_{3} & \leq C\left(\frac{\left(\mu\left(B_{6 r}\left(x_{1}\right)\right)\right)^{s_{2}}}{\lambda\left(\mathbf{L}_{\alpha_{1}, s_{1}}^{2 R}[\mu]\left(x_{1}\right)\right)^{-1}}\right)^{\frac{N}{s_{2}\left(N-\alpha_{2}\right)}} \\
& \leq C\left(\frac{\mathbf{L}_{\alpha_{1}, s_{1}}^{2 R}[\mu]\left(x_{1}\right) \mathbf{L}_{\alpha_{2}, s_{2}}^{2 R}[\mu]\left(x_{1}\right)}{\lambda}\right)^{\frac{N}{s_{2}\left(N-\alpha_{2}\right)}} r^{N} \\
& \leq C \varepsilon^{\frac{N}{2 s_{2}\left(N-\alpha_{2}\right)}}\left|B_{r}(x)\right| .
\end{aligned}
$$

Combining these inequalities, we infer (2.26).

Proof of Theorem 2.5. Step 1: Proof of (2.16). By [7, Theorem 2.3], we have

$$
\int_{\mathbb{R}^{N}}\left(\mathbf{M}_{\frac{\alpha p q_{1}+\beta p q_{2}}{q_{1}+q_{2}}}[\mu](x)\right)^{\frac{q_{1}+q_{2}}{p-1}} d x \asymp \int_{\mathbb{R}^{N}}\left(\mathbf{W}_{\frac{\alpha p q_{1}+\beta p q_{2}}{q_{1}+q_{2}}, p}[\mu](x)\right)^{q_{1}+q_{2}} d x .
$$

Next, we prove

$$
\int_{\mathbb{R}^{N}}\left(\mathbf{M}_{\frac{\alpha p q_{1}+\beta p q_{2}}{q_{1}+q_{2}}}[\mu](x)\right)^{\frac{q_{1}+q_{2}}{p-1}} d x \asymp \int_{\mathbb{R}^{N}}\left(\mathbf{W}_{\alpha, p}[\mu](x)\right)^{q_{1}}\left(\mathbf{W}_{\beta, p}[\mu](x)\right)^{q_{2}} d x .
$$

Since for all $x \in \mathbb{R}^{N}$ there holds

$$
\begin{aligned}
& \left(\mathbf{W}_{\alpha, p}[\mu](x)\right)^{q_{1}}\left(\mathbf{W}_{\beta, p}[\mu](x)\right)^{q_{2}} \geq C\left(\mathbf{M}_{\frac{\alpha p q_{1}+\beta p q_{2}}{q_{1}+q_{2}}}[\mu](x)\right)^{\frac{q_{1}+q_{2}}{p-1}}, \\
& \left(\mathbf{W}_{\alpha, p}[\mu](x)\right)^{q_{1}} \leq C\left(\mathbf{L}_{\alpha p, \frac{q_{1}}{\left(q_{1}+q_{2}\right)(p-1)}}[\mu](x)\right)^{q_{1}+q_{2}}, \\
& \left(\mathbf{W}_{\beta, p}[\mu](x)\right)^{q_{2}} \leq C\left(\mathbf{L}_{\beta p, \frac{q_{2}}{\left(q_{1}+q_{2}\right)(p-1)}}[\mu](x)\right)^{q_{1}+q_{2}},
\end{aligned}
$$

It is therefore enough to show that

$$
\int_{\mathbb{R}^{N}}\left(\mathbf{L}_{\alpha p, \frac{q_{1}}{\left(q_{1}+q_{2}\right)(p-1)}}[\mu] \mathbf{L}_{\beta p, \frac{q_{2}}{\left(q_{1}+q_{2}\right)(p-1)}}[\mu]\right)^{q_{1}+q_{2}} d x \leq C \int_{\mathbb{R}^{N}}\left(\mathbf{M}_{\frac{\alpha p q_{1}+\beta p q_{2}}{q_{1}+q_{2}}}[\mu]\right)^{\frac{q_{1}+q_{2}}{p-1}} d x .
$$

Set $d \mu_{n}=\chi_{B_{n}(0)} d \mu$, then we have

$$
\left|\left\{\mathbf{L}_{\alpha p, \frac{q_{1}}{\left(q_{1}+q_{2}\right)(p-1)}}\left[\mu_{n}\right] \mathbf{L}_{\beta p, \frac{q_{2}}{\left(q_{1}+q_{2}\right)(p-1)}}\left[\mu_{n}\right]>t\right\}\right|<\infty \text { for all } t>0 .
$$

Hence, by Lemma (2.6), there exist positive constants $C, \varepsilon_{0}, a$ such that for any $\lambda>0, \varepsilon \in$ $\left(0, \varepsilon_{0}\right)$

$$
\begin{aligned}
&\left|\left\{\mathbf{L}_{\alpha p, \frac{q_{1}}{\left(q_{1}+q_{2}\right)(p-1)}}\left[\mu_{n}\right] \mathbf{L}_{\beta p, \frac{q_{2}}{\left(q_{1}+q_{2}\right)(p-1)}}\left[\mu_{n}\right]>a \lambda\right\}\right| \\
& \leq C \varepsilon^{\frac{N\left(q_{1}+q_{2}\right)(p-1)}{2 q_{2}(N-\beta p)}}\left|\left\{\mathbf{L}_{\alpha p, \frac{q_{1}}{\left(q_{1}+q_{2}\right)(p-1)}}\left[\mu_{n}\right] \mathbf{L}_{\beta p, \frac{q_{2}}{\left(q_{1}+q_{2}\right)(p-1)}}\left[\mu_{n}\right]>\varepsilon^{1 / 2} \lambda\right\}\right| \\
&+\mid\left\{\left(\mathbf{M}_{\left.\left.\frac{\alpha p q_{1}+\beta p q_{2}}{q_{1}+q_{2}}[\mu]\right)^{\frac{1}{p-1}}>\varepsilon \lambda\right\} \mid .}\right.\right.
\end{aligned}
$$


Multiplying by $\lambda^{q_{1}+q_{2}-1}$ and integrating over $(0, \infty)$, we get

$$
\begin{gathered}
\int_{0}^{\infty} \lambda^{q_{1}+q_{2}}\left|\left\{\mathbf{L}_{\alpha p, \frac{q_{1}}{\left(q_{1}+q_{2}\right)(p-1)}}\left[\mu_{n}\right] \mathbf{L}_{\beta p, \frac{q_{2}}{\left(q_{1}+q_{2}\right)(p-1)}}\left[\mu_{n}\right]>a \lambda\right\}\right| \frac{d \lambda}{\lambda} \\
\leq C \varepsilon^{\frac{N\left(q_{1}+q_{2}\right)(p-1)}{2 q_{2}(N-\beta p)}} \int_{0}^{\infty} \lambda^{q_{1}+q_{2}}\left|\left\{\mathbf{L}_{\alpha p, \frac{q_{1}}{\left(q_{1}+q_{2}\right)(p-1)}}\left[\mu_{n}\right] \mathbf{L}_{\beta p, \frac{q_{2}}{\left(q_{1}+q_{2}\right)(p-1)}}\left[\mu_{n}\right]>\varepsilon^{1 / 2} \lambda\right\}\right| \frac{d \lambda}{\lambda} \\
+\int_{0}^{\infty} \lambda^{q_{1}+q_{2}}\left|\left\{\left(\mathbf{M}_{\frac{\alpha p q_{1}+\beta p q_{2}}{q_{1}+q_{2}}}[\mu]\right)^{\frac{1}{p-1}}>\varepsilon \lambda\right\}\right| \frac{d \lambda}{\lambda} .
\end{gathered}
$$

By a change of variable, we derive

$$
\begin{aligned}
& \left(a^{-q_{1}-q_{2}}-C \varepsilon^{\frac{N\left(q_{1}+q_{2}\right)(p-1)}{2 q_{2}(N-\beta p)}-\frac{q_{1}+q_{2}}{2}}\right) \\
& \quad \times \int_{0}^{\infty} \lambda^{q_{1}+q_{2}}\left|\left\{\mathbf{L}_{\alpha p, \frac{q_{1}}{\left(q_{1}+q_{2}\right)(p-1)}}\left[\mu_{n}\right] \mathbf{L}_{\beta p, \frac{q_{2}}{\left(q_{1}+q_{2}\right)(p-1)}}\left[\mu_{n}\right]>\lambda\right\}\right| \frac{d \lambda}{\lambda} \\
& \leq \varepsilon^{-q_{1}-q_{2}} \int_{0}^{\infty} \lambda^{q_{1}+q_{2}}\left|\left\{\left(\mathbf{M}_{\frac{\alpha p q_{1}+\beta p q_{2}}{q_{1}+q_{2}}}[\mu]\right)^{\frac{1}{p-1}}>\lambda\right\}\right| \frac{d \lambda}{\lambda} .
\end{aligned}
$$

Since $\frac{N\left(q_{1}+q_{2}\right)(p-1)}{2 q_{2}(N-\beta p)}-\frac{q_{1}+q_{2}}{2}>0$, there exists $\varepsilon_{0}>0$ such that for any $0<\epsilon \leq \epsilon_{0}$, there holds $a^{-q_{1}-q_{2}}-C \varepsilon^{\frac{N\left(q_{1}+q_{2}\right)(p-1)}{2 q_{2}(N-\beta p)}-\frac{q_{1}+q_{2}}{2}}>0$. Hence we obtain $(2.31)$ by Fatou's Lemma.

Step 2: Proof of (2.17). By [7, Theorem 2.3], we have

$$
\int_{\mathbb{R}^{N}}\left(\mathbf{M}_{\frac{\alpha p q_{1}+\beta p q_{2}}{q_{1}+q_{2}}}^{2 R}[\omega](x)\right)^{\frac{q_{1}+q_{2}}{p-1}} d x \asymp \int_{\mathbb{R}^{N}}\left(\mathbf{W}_{\frac{\alpha p q_{1}+\beta p q_{2}}{q_{1}+q_{2}}, p}^{2 R}[\omega](x)\right)^{q_{1}+q_{2}} d x .
$$

Next, we prove

$$
\int_{\mathbb{R}^{N}}\left(\mathbf{M}_{\frac{\alpha p q_{1}+\beta p q_{2}}{q_{1}+q_{2}}}^{2 R}[\omega](x)\right)^{\frac{q_{1}+q_{2}}{p-1}} d x \asymp \int_{\mathbb{R}^{N}}\left(\mathbf{W}_{\alpha, p}^{2 R}[\omega](x)\right)^{q_{1}}\left(\mathbf{W}_{\beta, p}^{2 R}[\omega](x)\right)^{q_{2}} d x .
$$

Let $x_{0} \in \mathbb{R}^{N}$ such that $\operatorname{supp}(\omega) \subset B_{R}\left(x_{0}\right)$. Since for all $x \in \mathbb{R}^{N}$,

$$
\left(\mathbf{W}_{\alpha, p}^{4 R}[\omega](x)\right)^{q_{1}}\left(\mathbf{W}_{\beta, p}^{4 R}[\omega](x)\right)^{q_{2}} \geq C\left(\mathbf{M}_{\frac{\alpha p q_{1}+\beta p q_{2}}{q_{1}+q_{2}}}^{2 R}[\omega](x)\right)^{\frac{q_{1}+q_{2}}{p-1}},
$$

and for any $y \in B_{3 R / 2}\left(x_{0}\right)$,

$$
\mathbf{W}_{\alpha, p}^{4 R}[\omega](y) \leq C \mathbf{W}_{\alpha, p}^{2 R}[\omega](y), \quad \mathbf{W}_{\beta, p}^{4 R}[\omega](y) \leq C \mathbf{W}_{\beta, p}^{2 R}[\omega](y),
$$

we have,

$$
\begin{aligned}
\int_{\mathbb{R}^{N}}\left(\mathbf{M}_{\left.\frac{\alpha p q+\beta p q_{2}}{q_{1}+q_{2}}[\omega](x)\right)^{\frac{q_{1}+q_{2}}{p-1}}}^{2 R} \leq C \int_{B_{5 R}\left(x_{0}\right)}\left(\mathbf{W}_{\alpha, p}^{4 R}[\omega](x)\right)^{q_{1}}\left(\mathbf{W}_{\beta, p}^{4 R}[\omega](x)\right)^{q_{2}} d x\right. \\
\leq C \int_{B_{3 R / 2}\left(x_{0}\right)}\left(\mathbf{W}_{\alpha, p}^{2 R}[\omega](x)\right)^{q_{1}}\left(\mathbf{W}_{\beta, p}^{2 R}[\omega](x)\right)^{q_{2}} d x \\
+C R^{N}\left(\frac{\omega\left(\mathbb{R}^{N}\right)}{R^{N-\frac{\alpha p q_{1}+\beta p q_{2}}{q_{1}+q_{2}}}}\right)^{\frac{q_{1}+q_{2}}{p-1}} \\
\leq C \int_{\mathbb{R}^{N}}\left(\mathbf{W}_{\alpha, p}^{2 R}[\omega](x)\right)^{q_{1}}\left(\mathbf{W}_{\beta, p}^{2 R}[\omega](x)\right)^{q_{2}} d x .
\end{aligned}
$$


On the other hand, since there holds almost everywhere,

$$
\left(\mathbf{W}_{\alpha, p}^{2 R}[\omega](x)\right)^{q_{1}} \leq C\left(\mathbf{L}_{\alpha p, \frac{q_{1}}{\left(q_{1}+q_{2}\right)(p-1)}}^{3 R}[\omega](x)\right)^{q_{1}+q_{2}},\left(\mathbf{W}_{\beta, p}^{2 R}[\omega](x)\right)^{q_{2}} \leq C\left(\mathbf{L}_{\beta p, \frac{q_{2}}{\left(q_{1}+q_{2}\right)(p-1)}}^{3 R}[\omega](x)\right)^{q_{1}+q_{2}}
$$

it is enough to prove that

$$
\int_{\mathbb{R}^{N}}\left(\mathbf{L}_{\alpha p, \frac{q_{1}}{3 R}\left[\left(q_{1}+q_{2}\right)(p-1)\right.}[\omega](x) \mathbf{L}_{\beta p, \frac{q_{2}}{\left(q_{1}+q_{2}\right)(p-1)}}^{3 R}[\omega](x)\right)^{q_{1}+q_{2}} d x \leq C \int_{\mathbb{R}^{N}}\left(\mathbf{M}_{\frac{\alpha p q_{1}+\beta p q_{2}}{q_{1}+q_{2}}}^{2 R}[\omega](x)\right)^{\frac{q_{1}+q_{2}}{p-1}} d x .
$$

By Lemma (2.6) there exist positive constants $C, \varepsilon_{0}$ and $a$ such that for any $\lambda>0, \varepsilon \in\left(0, \varepsilon_{0}\right)$,

$$
\begin{aligned}
& \left|\left\{\mathbf{L}_{\alpha p, \frac{q_{1}}{3 R}}^{3 R}[\omega] \mathbf{L}_{\beta p, \frac{q_{2}}{\left(q_{1}+q_{2}\right)(p-1)}}^{3 R}[\omega]>a \lambda\right\}\right| \\
& \leq C \varepsilon^{\frac{N\left(q_{1}+q_{2}\right)(p-1)}{2 q_{2}(N-\beta p)}}\left|\left\{\mathbf{L}_{\alpha p, \frac{q_{1}}{\left(q_{1}+q_{2}\right)(p-1)}}^{6 R}[\omega] \mathbf{L}_{\beta p, \frac{q_{2}}{\left(q_{1}+q_{2}\right)(p-1)}}^{6 R}[\omega]>\varepsilon^{1 / 2} \lambda\right\}\right| \\
& \quad+\left|\left\{\left(\mathbf{M}_{\frac{\alpha p q_{1}+\beta p q_{2}}{q_{1}+q_{2}}}^{6 R}[\omega]\right)^{\frac{1}{p-1}}>\varepsilon \lambda\right\}\right| .
\end{aligned}
$$

Multiplying by $\lambda^{q_{1}+q_{2}-1}$ and integrating over $(0, \infty)$, we obtain

$$
\begin{gathered}
a^{-q_{1}-q_{2}} \int_{\mathbb{R}^{N}}\left(\mathbf{L}_{\alpha p, \frac{q_{1}}{\left(q_{1}+q_{2}\right)(p-1)}}^{3 R}[\omega](x) \mathbf{L}_{\beta p, \frac{q_{2}}{\left(q_{1}+q_{2}\right)(p-1)}}^{3 R}[\omega](x)\right)^{q_{1}+q_{2}} d x \\
\leq C \varepsilon^{\frac{N\left(q_{1}+q_{2}\right)(p-1)}{2 q_{2}(N-\beta p)}-\frac{q_{1}+q_{2}}{2}} \int_{\mathbb{R}^{N}}\left(\mathbf{L}_{\alpha p, \frac{q_{1}}{\left(q_{1}+q_{2}\right)(p-1)}}^{6 R}[\omega](x) \mathbf{L}_{\beta p, \frac{q_{2}}{\left(q_{1}+q_{2}\right)(p-1)}}^{6 R}[\omega](x)\right)^{q_{1}+q_{2}} d x \\
+\varepsilon^{-q_{1}-q_{2}} \int_{\mathbb{R}^{N}}\left(\mathbf{M}_{\frac{\alpha p q_{1}+\beta p q_{2}}{q_{1}+q_{2}}}^{6 R}[\omega](x)\right)^{\frac{q_{1}+q_{2}}{p-1}} d x .
\end{gathered}
$$

Similarly as (2.32), we can see that

$$
\begin{aligned}
\int_{\mathbb{R}^{N}} & \left(\mathbf{L}_{\alpha p, \frac{q_{1}}{\left(q_{1}+q_{2}\right)(p-1)}}^{6 R}[\omega](x) \mathbf{L}_{\beta p, \frac{q_{2}}{\left(q_{1}+q_{2}\right)(p-1)}}^{6 R}[\omega](x)\right)^{q_{1}+q_{2}} d x \\
& \leq C \int_{\mathbb{R}^{N}}\left(\mathbf{L}_{\alpha p, \frac{q_{1}}{\left(q_{1}+q_{2}\right)(p-1)}}^{2 R}[\omega](x) \mathbf{L}_{\beta p, \frac{q_{2}}{\left(q_{1}+q_{2}\right)(p-1)}}^{2 R}[\omega](x)\right)^{q_{1}+q_{2}} d x
\end{aligned}
$$

and

$$
\int_{\mathbb{R}^{N}}\left(\mathbf{M}_{\frac{\alpha p q_{1}+\beta p q_{2}}{q_{1}+q_{2}}}^{6 R}[\omega](x)\right)^{\frac{q_{1}+q_{2}}{p-1}} d x \leq \int_{\mathbb{R}^{N}}\left(\mathbf{M}_{\frac{\alpha p q_{1}+\beta p q_{2}}{q_{1}+q_{2}}}^{2 R}[\omega](x)\right)^{\frac{q_{1}+q_{2}}{p-1}} d x
$$

Therefore, since $\frac{N\left(q_{1}+q_{2}\right)(p-1)}{2 q_{2}(N-\beta p)}-\frac{q_{1}+q_{2}}{2}>0$, for some $\varepsilon>0$ small enough we infer (2.33).

Lemma 2.9 Let $\alpha>0, p>1,0<\alpha p<N$ and $0<\gamma<\frac{N(p-1)}{N-\alpha p}$. There exists a constant $C=C(N, \alpha, p, \gamma)$ such that for any $\mu \in \mathfrak{M}^{+}\left(\mathbb{R}^{N}\right)$,

$$
\int_{B_{r}(x)}\left(\mathbf{W}_{\alpha, p}^{r}[\mu]\right)^{\gamma} d y \leq C r^{N}\left(\frac{\mu\left(B_{2 r}(x)\right)}{r^{N-\alpha p}}\right)^{\frac{\gamma}{p-1}} \text { for all } x \in \mathbb{R}^{N} \text { and } r>0 .
$$


Proof. We have

$$
\begin{aligned}
\int_{B_{r}(x)}\left(\mathbf{W}_{\alpha, p}^{r}[\mu]\right)^{\gamma} d y & \leq \int_{B_{r}(x)}\left(\mathbf{W}_{\alpha, p}\left[\chi_{B_{2 r}(x)} \mu\right]\right)^{\gamma} d y \\
& =\gamma \int_{0}^{\infty} \lambda^{\gamma-1}\left|\left\{\mathbf{W}_{\alpha, p}\left[\chi_{B_{2 r}(x)} \mu\right]>\lambda\right\} \cap B_{r}(x)\right| d \lambda .
\end{aligned}
$$

By Lemma 2.7, we obtain

$$
\begin{gathered}
\int_{B_{r}(x)}\left(\mathbf{W}_{\alpha, p}^{r}[\mu]\right)^{\gamma} d y \leq \gamma\left(\frac{\mu\left(B_{2 r}(x)\right)}{r^{N-\alpha p}}\right)^{\frac{\gamma}{p-1}}\left|B_{r}(x)\right| \\
\left.\quad+\int_{\left(\frac{\mu\left(B_{2 r}(x)\right)}{r^{N-\alpha p}}\right)^{\frac{1}{p-1}} \lambda^{\gamma-1}\left|\left\{\mathbf{W}_{\alpha, p}\left[\chi_{B_{2 r}(x)} \mu\right]>\lambda\right\}\right| d \lambda}^{\infty}\right)_{\leq} C^{N}\left(\frac{\mu\left(B_{2 r}(x)\right)}{r^{N-\alpha p}}\right)^{\frac{\gamma}{p-1}}+C \int_{\left(\frac{\mu\left(B_{2 r}(x)\right)}{r^{N-\alpha p}}\right)^{\frac{1}{p-1}} \lambda^{\gamma-1}\left(\frac{\left(\mu\left(B_{2 r}(x)\right)\right)^{\frac{1}{p-1}}}{\lambda}\right)^{\frac{N(p-1)}{N-\alpha p}} d \lambda}^{\infty} d r^{N}\left(\frac{\mu\left(B_{2 r}(x)\right)}{r^{N-\alpha p}}\right)^{\frac{\gamma}{p-1}} .
\end{gathered}
$$

which is the claim.

The next result is fundamental inasmuch it shows the equivalence between the capacitary estimates, the potential inequalities used in our construction and the solvability of the system of nonlinear integral equations connected to (1.1).

Theorem 2.10 Let $\alpha, \beta, q_{1}, q_{2}>0, \alpha>\beta, 1<p<\min \{N / \alpha, N / \beta\}, q_{1}+q_{2}>p-1$, $q_{2}<\frac{N(p-1)}{N-\beta p}$ and $\frac{\alpha p q_{1}+\beta p q_{2}}{q_{1}+q_{2}}<N$ and $\mu \in \mathfrak{M}^{+}\left(\mathbb{R}^{N}\right)$. Then, the following statements are equivalent:

(a) The inequality

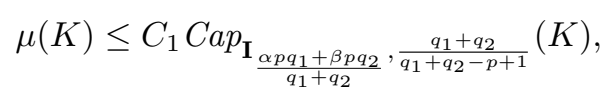

holds for any compact set $K \subset \mathbb{R}^{N}$, for some $C_{1}>0$.

(b) The inequality

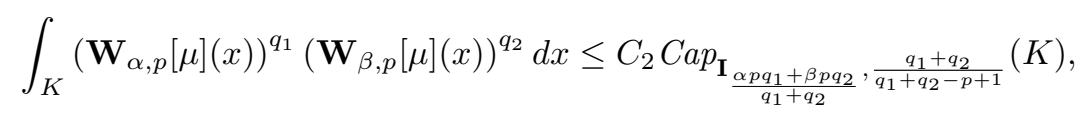

holds for any compact set $K \subset \mathbb{R}^{N}$, for some $C_{2}>0$.

(c) The inequality

$$
\int_{\mathbb{R}^{N}}\left(\mathbf{W}_{\frac{\alpha q_{1}+\beta q_{2}}{q_{1}+q_{2}}, p}\left[\chi_{B_{t}(x)} \mu\right](y)\right)^{q_{1}+q_{2}} d y \leq C_{3} \mu\left(B_{t}(x)\right),
$$

holds for any ball $B_{t}(x) \subset \mathbb{R}^{N}$, for some $C_{3}>0$.

(d) The inequality

$$
\int_{\mathbb{R}^{N}}\left(\mathbf{W}_{\alpha, p}\left[\chi_{B_{t}(x)} \mu\right](y)\right)^{q_{1}}\left(\mathbf{W}_{\beta, p}\left[\chi_{B_{t}(x)} \mu\right](y)\right)^{q_{2}} d y \leq C_{4} \mu\left(B_{t}(x)\right),
$$

holds for any ball $B_{t}(x) \subset \mathbb{R}^{N}$, for some $C_{4}>0$. 
(e) The inequalities

$$
\begin{aligned}
& \mathbf{W}_{\alpha, p}\left[\left(\mathbf{W}_{\alpha, p}[\mu]\right)^{q_{1}}\left(\mathbf{W}_{\beta, p}[\mu]\right)^{q_{2}}\right] \leq C_{5} \mathbf{W}_{\alpha, p}[\mu]<\infty \\
& \mathbf{W}_{\beta, p}\left[\left(\mathbf{W}_{\alpha, p}[\mu]\right)^{q_{1}}\left(\mathbf{W}_{\beta, p}[\mu]\right)^{q_{2}}\right] \leq C_{5} \mathbf{W}_{\beta, p}[\mu]<\infty
\end{aligned}
$$

hold for some $C_{5}>0$.

(f) The system equation

$$
\begin{aligned}
& U=\mathbf{W}_{\alpha, p}\left[U^{q_{1}} V^{q_{2}}\right]+\varepsilon \mathbf{W}_{\alpha, p}[\mu] \\
& V=\mathbf{W}_{\beta, p}\left[U^{q_{1}} V^{q_{2}}\right]+\varepsilon \mathbf{W}_{\beta, p}[\mu],
\end{aligned}
$$

in $\mathbb{R}^{N}$ has a nonnegative solution for some $\varepsilon>0$.

Proof. By Theorem 2.1 we have (a) $\Leftrightarrow$ (c), by Theorem 2.5, (c) $\Leftrightarrow$ (d). We now assume $(e)$. Put $\mathbf{T}[\mu]=\left(\mathbf{W}_{\alpha, p}[\mu](x)\right)^{q_{1}}\left(\mathbf{W}_{\beta, p}[\mu](x)\right)^{q_{2}}$ for any $\mu \in \mathfrak{M}^{+}\left(\mathbb{R}^{N}\right)$. It is easy to see that

$$
(\mathbf{T}[\mu](x))^{\gamma} \geq C \int_{0}^{\infty}\left(\frac{\mu\left(B_{\rho}(x)\right)}{\rho^{N-\frac{\alpha q_{1} p+\beta q_{2} p}{q_{1}+q_{2}}}}\right)^{\frac{\gamma\left(q_{1}+q_{2}\right)}{p-1}} \frac{d \rho}{\rho}=C \mathbf{W}_{\beta, s}[\mu](x) \text { for all } x \in \mathbb{R}^{N}
$$

where $\gamma=\frac{p-1}{q_{1}}+\frac{p-1}{q_{2}}, \beta=\frac{\gamma\left(\alpha q_{1} p+\beta q_{2} p\right)}{\gamma\left(q_{1}+q_{2}\right)+p-1}$ and $s=\frac{\gamma\left(q_{1}+q_{2}\right)+p-1}{\gamma\left(q_{1}+q_{2}\right)}<1+\frac{1}{\gamma}$. From (2.39) and (2.40), we have

$$
\mathbf{T}[\mathbf{T}[\mu]] \leq C \mathbf{T}[\mu]<\infty \text { almost everywhere. }
$$

Using (2.42), we obtain

$$
\left(\mathbf{W}_{\beta, s}[\mathbf{T}[\mu]]\right)^{\frac{1}{\gamma}} \leq C \mathbf{T}[\mu]<\infty \text { almost everywhere. }
$$

Applying $\mathbf{W}_{\beta, s}$ to both sides of the above inequality and using Theorem 2.1 with $\alpha=\beta, p=$ $s, q=\frac{1}{\gamma}$, we derive

$$
\int_{K} \mathbf{T}[\mu](x) d x \leq C \operatorname{Cap}_{\mathbf{I}_{\beta s}, \frac{1}{1+\gamma-\gamma s}}(K),
$$

for any compact set $K \subset \mathbb{R}^{N}$, which implies (b). So, (e) $\Rightarrow$ (b). Next, assume (b), using (2.42) again, we derive from (b) that

$$
\int_{K}\left(\mathbf{W}_{\beta, s}[\mu](x)\right)^{\frac{1}{\gamma}} d x \leq C \operatorname{Cap}_{\mathbf{I}_{\beta s}, \frac{1}{1+\gamma-\gamma s}}(K),
$$

for any compact set $K \subset \mathbb{R}^{N}$. Thanks to Theorem 2.1, we get (a). So, (b) $\Rightarrow$ (a). It remains to prove that (i): (f) $\Rightarrow$ (a), (ii): (e) $\Rightarrow$ (f), (iii): (a)+(c)+(d) $\Rightarrow$ (e).

(i): Assume that (2.41) has a nonnegative solution for some $\varepsilon>0$. Set $d \nu(x)=U^{q_{1}} V^{q_{2}} d x+$ $\varepsilon d \mu(x)$. Clearly

$$
\left(\mathbf{W}_{\alpha, p}[\nu]\right)^{q_{1}}\left(\mathbf{W}_{\beta, p}[\nu]\right)^{q_{2}} \leq C d \nu(x) \text { in } \mathbb{R}^{N} .
$$

If $E \subset \mathbb{R}^{N}$ is a Borel set, we have

$$
\begin{aligned}
\int_{\mathbb{R}^{N}}\left(\mathbf{W}_{\alpha, p}\left[\chi_{E} \nu\right]\right)^{q_{1}}\left(\mathbf{W}_{\beta, p}\left[\chi_{E} \nu\right]\right)^{q_{2}} d x & \leq \int_{\mathbb{R}^{N}}\left(\mathbf{M}_{\nu} \chi_{E}\right)^{\frac{q_{1}+q_{2}}{p-1}}\left(\mathbf{W}_{\alpha, p}[\nu]\right)^{q_{1}}\left(\mathbf{W}_{\beta, p}[\nu]\right)^{q_{2}} d x \\
& \leq C \int_{\mathbb{R}^{N}}\left(\mathbf{M}_{\nu} \chi_{E}\right)^{\frac{q_{1}+q_{2}}{p-1}} d \nu .
\end{aligned}
$$


Since $M_{\omega} f$ is bounded on $L^{s}\left(\mathbb{R}^{N}, d \omega\right), s>1$, we deduce from Fefferman's result [11] that

$$
\int_{\mathbb{R}^{N}}\left(\mathbf{W}_{\alpha, p}\left[\chi_{E} \nu\right]\right)^{q_{1}}\left(\mathbf{W}_{\beta, p}\left[\chi_{E} \nu\right]\right)^{q_{2}} d x \leq C \nu(E),
$$

is verified for any Borel set $E \subset \mathbb{R}^{N}$. Applying (a) $\Leftrightarrow$ (c) to $\mu=\nu$, we derive that

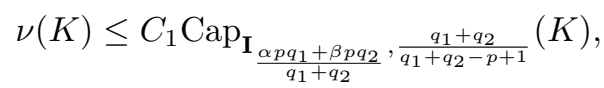

holds for any compact set $K \subset \mathbb{R}^{N}$. Since $\nu \geq \mu$, we obtain (c).

(ii): Suppose that (2.39) and (2.40) hold with constant $C_{5}>0$. Take $0<\varepsilon \leq \frac{1}{2\left(2 C_{5}\right)^{\frac{p-1}{q_{1}+q_{2}-p+1}}}$. Consider the sequence $\left\{U_{m}, V_{m}\right\}_{m \geq 0}$ of nonnegative functions defined by $U_{0}=\mathbf{W}_{\alpha, p}[\mu], V_{0}=$ $\mathbf{W}_{\beta, p}[\mu]$ and

$$
\begin{aligned}
& U_{m+1}=\mathbf{W}_{\alpha, p}\left[U_{m}^{q_{1}} V_{m}^{q_{2}}\right]+\varepsilon \mathbf{W}_{\alpha, p}[\mu] \\
& V_{m+1}=\mathbf{W}_{\beta, p}\left[U_{m}^{q_{1}} V_{m}^{q_{2}}\right]+\varepsilon \mathbf{W}_{\beta, p}[\mu] .
\end{aligned}
$$

It is easy to see that $\left\{U_{m}, V_{m}\right\}_{m \geq 0}$ is well defined and satisfies

$$
U_{m} \leq 2 \varepsilon \mathbf{W}_{\alpha, p}[\mu], V_{m} \leq 2 \varepsilon \mathbf{W}_{\alpha, p}[\mu] \text { for all } m \geq 0 .
$$

Clearly $\left\{U_{m}\right\},\left\{V_{m}\right\}$ are nondecreasing. Using the dominated convergence theorem, it follows that $(U(x), V(x)):=\lim _{m \rightarrow \infty}\left(U_{m}(x), V_{m}(x)\right)$ is a solution of $(2.41)$.

(iii): Assume that statements (a), (c) and (d) hold true. We first assume that $\mu$ has compact support. From (a) we have

$$
\mu\left(B_{r}(x)\right) \leq C r^{N-\frac{\alpha p q_{1}+\beta p q_{2}}{q_{1}+q_{2}-p+1}} \text { for all } x \in \mathbb{R}^{N} \text { and } r>0 .
$$

From (b)

$$
\int_{B_{r}(x)}\left(\mathbf{W}_{\frac{\alpha q_{1}+\beta q_{2}}{q_{1}+q_{2}}, p}^{r}[\mu](y)\right)^{q_{1}+q_{2}} d y \leq C_{2} \mu\left(B_{2 r}(x)\right) \text { for all } x \in \mathbb{R}^{N} \text { and } r>0 .
$$

Using Hölder's inequality and $\mathbf{W}_{\frac{\alpha q_{1}+\beta q_{2}}{q_{1}+q_{2}}, p}^{r}[\mu] \geq r^{-\frac{(\alpha-\beta) p q_{2}}{(p-1)\left(q_{1}+q_{2}\right)}} \mathbf{W}_{\alpha, p}^{r}[\mu]$, we obtain,

$$
\int_{B_{r}(x)}\left(\mathbf{W}_{\alpha, p}^{r}[\mu](y)\right)^{q_{1}} d y \leq C r^{\frac{(\alpha-\beta) p q_{1} q_{2}+(p-1) N q_{2}+(N-\beta p)(p-1) q_{1}}{(p-1)\left(q_{1}+q_{2}\right)}}\left(\frac{\mu\left(B_{2 r}(x)\right)}{r^{N-\beta p}}\right)^{\frac{q_{1}}{q_{1}+q_{2}}}
$$

again for all $x \in \mathbb{R}^{N}$ and $r>0$. From (c),

$$
\int_{B_{r}(x)}\left(\mathbf{W}_{\alpha, p}^{r}[\mu](y)\right)^{q_{1}}\left(\mathbf{W}_{\beta, p}^{r}[\mu](y)\right)^{q_{2}} d y \leq C_{3} \mu\left(B_{2 r}(x)\right) \text { for all } x \in \mathbb{R}^{N} \text { and } r>0 \text {. }
$$

By Lemma 2.9,

$$
\int_{B_{r}(x)}\left(\mathbf{W}_{\beta, p}^{r}[\mu]\right)^{q_{2}} d y \leq C r^{N}\left(\frac{\mu\left(B_{2 r}(x)\right)}{r^{N-\beta p}}\right)^{\frac{q_{2}}{p-1}} \text { for all } x \in \mathbb{R}^{N} \text { and } r>0 .
$$

We have, with $\eta=\alpha$ or $\eta=\beta$,

$$
\mathbf{W}_{\eta, p}\left[\left(\mathbf{W}_{\alpha, p}[\mu]\right)^{q_{1}}\left(\mathbf{W}_{\beta, p}[\mu]\right)^{q_{2}}\right](x) \leq C \sum_{i=1}^{4} \int_{0}^{\infty}\left(\frac{\mathbf{A}_{i}(x, r)}{r^{N-\eta p}}\right)^{\frac{1}{p-1}} \frac{d r}{r}
$$


where

$$
\begin{aligned}
& \mathbf{A}_{1}(x, r)=\int_{B_{r}(x)}\left(\mathbf{W}_{\alpha, p}^{r}[\mu](y)\right)^{q_{1}}\left(\mathbf{W}_{\beta, p}^{r}[\mu](y)\right)^{q_{2}} d y \\
& \mathbf{A}_{2}(x, r)=\int_{B_{r}(x)}\left(\mathbf{W}_{\alpha, p}^{r}[\mu](y)\right)^{q_{1}}\left(\int_{r}^{\infty}\left(\frac{\mu\left(B_{t}(y)\right)}{t^{N-\beta p}}\right)^{\frac{1}{p-1}} \frac{d t}{t}\right)^{q_{2}} d y, \\
& \mathbf{A}_{3}(x, r)=\int_{B_{r}(x)}\left(\int_{r}^{\infty}\left(\frac{\mu\left(B_{t}(y)\right)}{t^{N-\alpha p}}\right)^{\frac{1}{p-1}} \frac{d t}{t}\right)^{q_{1}}\left(\mathbf{W}_{\beta, p}^{r}[\mu](y)\right)^{q_{2}} d y, \\
& \mathbf{A}_{4}(x, r)=\int_{B_{r}(x)}\left(\int_{r}^{\infty}\left(\frac{\mu\left(B_{t}(y)\right)}{t^{N-\alpha p}}\right)^{\frac{1}{p-1}} \frac{d t}{t}\right)^{q_{1}}\left(\int_{r}^{\infty}\left(\frac{\mu\left(B_{t}(y)\right)}{t^{N-\beta p}}\right)^{\frac{1}{p-1}} \frac{d t}{t}\right)^{q_{2}} d y .
\end{aligned}
$$

Thanks to (2.47) we get

$$
\mathbf{A}_{1}(x, r) \leq C \mu\left(B_{2 r}(x)\right)
$$

which implies

$$
\int_{0}^{\infty}\left(\frac{\mathbf{A}_{1}(x, r)}{r^{N-\eta p}}\right)^{\frac{1}{p-1}} \frac{d r}{r} \leq C \mathbf{W}_{\eta, p}[\mu](x) .
$$

Since $B_{t}(y) \leq B_{2 t}(x)$ for any $y \in B_{r}(x), t \geq r$ and thanks to (2.46), (2.9) we deduce

$$
\begin{aligned}
& \mathbf{A}_{2}(x, t) \leq \int_{B_{r}(x)}\left(\mathbf{W}_{\alpha, p}^{r}[\mu](y)\right)^{q_{1}} d y\left(\int_{r}^{\infty}\left(\frac{\mu\left(B_{2 t}(x)\right)}{t^{N-\beta p}}\right)^{\frac{1}{p-1}} \frac{d t}{t}\right)^{q_{2}} \\
& \leq C r^{\frac{(\alpha-\beta) p q_{1} q_{2}+(p-1) N q_{2}+(N-\beta p)(p-1) q_{1}}{(p-1)\left(q_{1}+q_{2}\right)}}\left(\frac{\mu\left(B_{2 r}(x)\right)}{r^{N-\beta p}}\right)^{\frac{q_{1}}{q_{1}+q_{2}}}\left(\int_{r}^{\infty}\left(\frac{\mu\left(B_{2 t}(x)\right)}{t^{N-\beta p}}\right)^{\frac{1}{p-1}} \frac{d t}{t}\right)^{q_{2}} \\
& \leq C r^{\frac{(\alpha-\beta) p q_{1} q_{2}+(p-1) N q_{2}+(N-\beta p)(p-1) q_{1}}{(p-1)\left(q_{1}+q_{2}\right)}}\left(\int_{r}^{\infty}\left(\frac{\mu\left(B_{2 t}(x)\right)}{t^{N-\beta p}}\right)^{\frac{1}{p-1}} \frac{d t}{t}\right)^{q_{2}+\frac{q_{1}(p-1)}{q_{1}+q_{2}}},
\end{aligned}
$$

then

$$
\begin{aligned}
\mathbf{A}_{3}(x, t) & \leq\left(\int_{r}^{\infty}\left(\frac{\mu\left(B_{2 t}(x)\right)}{t^{N-\alpha p}}\right)^{\frac{1}{p-1}} \frac{d t}{t}\right)^{q_{1}} \int_{B_{r}(x)}\left(\mathbf{W}_{\beta, p}^{r}[\mu](y)\right)^{q_{2}} d y \\
& \leq C\left(\int_{r}^{\infty}\left(\frac{\mu\left(B_{2 t}(x)\right)}{t^{N-\alpha p}}\right)^{\frac{1}{p-1}} \frac{d t}{t}\right)^{q_{1}} r^{N}\left(\frac{\mu\left(B_{2 r}(x)\right)}{r^{N-\beta p}}\right)^{\frac{q_{2}}{p-1}} \\
& \leq C r^{N}\left(\int_{r}^{\infty}\left(\frac{\mu\left(B_{2 t}(x)\right)}{t^{N-\alpha p}}\right)^{\frac{1}{p-1}} \frac{d t}{t}\right)^{q_{1}}\left(\int_{r}^{\infty}\left(\frac{\mu\left(B_{2 t}(x)\right)}{t^{N-\beta p}}\right)^{\frac{1}{p-1}} \frac{d t}{t}\right)^{q_{2}},
\end{aligned}
$$

and finally

$$
\mathbf{A}_{4}(x, t) \leq C r^{N}\left(\int_{r}^{\infty}\left(\frac{\mu\left(B_{2 t}(x)\right)}{t^{N-\alpha p}}\right)^{\frac{1}{p-1}} \frac{d t}{t}\right)^{q_{1}}\left(\int_{r}^{\infty}\left(\frac{\mu\left(B_{2 t}(x)\right)}{t^{N-\beta p}}\right)^{\frac{1}{p-1}} \frac{d t}{t}\right)^{q_{2}}
$$


I- From the estimate of $\mathbf{A}_{2}$ we derive

$$
\begin{aligned}
\int_{0}^{\infty}\left(\frac{\mathbf{A}_{2}(x, r)}{r^{N-\eta p}}\right)^{\frac{1}{p-1}} \frac{d r}{r} & \leq C \int_{0}^{\infty} r^{\frac{(\alpha-\beta) p q_{1} q_{2}+(p-1) N q_{2}+(N-\beta p)(p-1) q_{1}}{(p-1)^{2}\left(q_{1}+q_{2}\right)}-\frac{N-\eta p}{p-1}} \\
& \times\left(\int_{r}^{\infty}\left(\frac{\mu\left(B_{2 t}(x)\right)}{t^{N-\beta p}}\right)^{\frac{1}{p-1}} \frac{d t}{t}\right)^{\frac{q_{2}}{p-1}+\frac{q_{1}}{q_{1}+q_{2}}} \frac{d r}{r}
\end{aligned}
$$

Since $\frac{\alpha p q_{1}+\beta p q_{2}}{q_{1}+q_{2}}<N$, it follows that

$$
\begin{aligned}
0<\kappa: & =\frac{(\alpha-\beta) p q_{1} q_{2}+(p-1) N q_{2}+(N-\beta p)(p-1) q_{1}}{(p-1)^{2}\left(q_{1}+q_{2}\right)}-\frac{N-\eta p}{p-1} \\
& <\frac{N-\beta p}{p-1}\left(\frac{q_{2}}{p-1}+\frac{q_{1}}{q_{1}+q_{2}}\right) .
\end{aligned}
$$

Hence,

$$
r^{\kappa}\left(\int_{r}^{\infty}\left(\frac{\mu\left(B_{2 t}(x)\right)}{t^{N-\beta p}}\right)^{\frac{1}{p-1}} \frac{d t}{t}\right)^{\frac{q_{2}}{p-1}+\frac{q_{1}}{q_{1}+q_{2}}} \rightarrow 0 \text { as } t \rightarrow 0
$$

and therefore

$$
\begin{aligned}
r^{\kappa}\left(\int_{r}^{\infty}\left(\frac{\mu\left(B_{2 t}(x)\right)}{t^{N-\beta p}}\right)^{\frac{1}{p-1}} \frac{d t}{t}\right)^{\frac{q_{2}}{p-1}+\frac{q_{1}}{q_{1}+q_{2}}} \\
\leq C r^{\kappa-\frac{N-\beta p}{p-1}\left(\frac{q_{2}}{p-1}+\frac{q_{1}}{q_{1}+q_{2}}\right)}\left(\mu\left(\mathbb{R}^{N}\right)\right)^{\frac{q_{2}}{(p-1)^{2}}+\frac{q_{1}}{(p-1)\left(q_{1}+q_{2}\right)}}
\end{aligned}
$$

a quantity which converges to 0 when $t \rightarrow 0$. Hence, by integration be parts, we obtain

$$
\begin{gathered}
\int_{0}^{\infty}\left(\frac{\mathbf{A}_{2}(x, r)}{r^{N-\eta p}}\right)^{\frac{1}{p-1}} \frac{d r}{r} \leq C \int_{0}^{\infty} r^{\kappa}\left(\int_{r}^{\infty}\left(\frac{\mu\left(B_{2 t}(x)\right)}{t^{N-\beta p}}\right)^{\frac{1}{p-1}} \frac{d t}{t}\right)^{\frac{q_{2}}{p-1}+\frac{q_{1}}{q_{1}+q_{2}}} \frac{d r}{r} \\
\leq C \int_{0}^{\infty} r^{\kappa}\left(\int_{r}^{\infty}\left(\frac{\mu\left(B_{2 t}(x)\right)}{t^{N-\beta p}}\right)^{\frac{1}{p-1}} \frac{d t}{t}\right)^{\frac{q_{2}}{p-1}+\frac{q_{1}}{q_{1}+q_{2}}-1}\left(\frac{\mu\left(B_{2 r}(x)\right)}{r^{N-\beta p}}\right)^{\frac{1}{p-1}} \frac{d r}{r} \\
=C \int_{0}^{\infty} r^{\frac{(\alpha-\beta) p q_{1} q_{2}+(p-1) N q_{2}+(N-\beta p)(p-1) q_{1}}{(p-1)^{2}\left(q_{1}+q_{2}\right)}-\frac{N-\beta p}{p-1}} \\
\quad \times\left(\int_{r}^{\infty}\left(\frac{\mu\left(B_{2 t}(x)\right)}{t^{N-\beta p}}\right)^{\frac{1}{p-1}} \frac{d t}{t}\right)^{\frac{q_{2}}{p-1}+\frac{q_{1}}{q_{1}+q_{2}}-1}\left(\frac{\mu\left(B_{2 r}(x)\right)}{r^{N-\eta p}}\right)^{\frac{1}{p-1}} \frac{d r}{r}
\end{gathered}
$$

Observing that we have from (2.45),

$$
r^{\frac{(\alpha-\beta) p q_{1} q_{2}+(p-1) N q_{2}+(N-\beta p)(p-1) q_{1}}{(p-1)^{2}\left(q_{1}+q_{2}\right)}-\frac{N-\beta p}{p-1}}\left(\int_{r}^{\infty}\left(\frac{\mu\left(B_{2 t}(x)\right)}{t^{N-\beta p}}\right)^{\frac{1}{p-1}} \frac{d t}{t}\right)^{\frac{q_{2}}{p-1}+\frac{q_{1}}{q_{1}+q_{2}}-1} \leq C,
$$

we derive

$$
\int_{0}^{\infty}\left(\frac{\mathbf{A}_{2}(x, r)}{r^{N-\eta p}}\right)^{\frac{1}{p-1}} \frac{d r}{r} \leq C \mathbf{W}_{\eta, p}[\mu](x)
$$


II- From the estimate of $\mathbf{A}_{3}$ and $\mathbf{A}_{4}$, we have, as above, by integration be parts,

$$
\begin{aligned}
& \int_{0}^{\infty}\left(\frac{\mathbf{A}_{3}(x, r)}{r^{N-\eta p}}\right)^{\frac{1}{p-1}} \frac{d r}{r}+\int_{0}^{\infty}\left(\frac{\mathbf{A}_{4}(x, r)}{r^{N-\eta p}}\right)^{\frac{1}{p-1}} \frac{d r}{r} \\
& \leq C \int_{0}^{\infty} r^{\frac{\eta p}{p-1}}\left(\int_{r}^{\infty}\left(\frac{\mu\left(B_{2 t}(x)\right)}{t^{N-\alpha p}}\right)^{\frac{1}{p-1}} \frac{d t}{t}\right)^{\frac{q_{1}}{p-1}}\left(\int_{r}^{\infty}\left(\frac{\mu\left(B_{2 t}(x)\right)}{t^{N-\beta p}}\right)^{\frac{1}{p-1}} \frac{d t}{t}\right)^{\frac{q_{2}}{p-1}} \frac{d r}{r} \\
& \quad=C \int_{0}^{\infty} \mathbf{D}_{1}(x, r)\left(\frac{\mu\left(B_{2 r}(x)\right)}{r^{N-\eta p}}\right)^{\frac{1}{p-1}} \frac{d r}{r}+C \int_{0}^{\infty} \mathbf{D}_{2}(x, r)\left(\frac{\mu\left(B_{2 r}(x)\right)}{r^{N-\eta p}}\right)^{\frac{1}{p-1}} \frac{d r}{r},
\end{aligned}
$$

where

$$
\begin{aligned}
& \mathbf{D}_{1}(x, r)=r^{\frac{\alpha p}{p-1}}\left(\int_{r}^{\infty}\left(\frac{\mu\left(B_{2 t}(x)\right)}{t^{N-\alpha p}}\right)^{\frac{1}{p-1}} \frac{d t}{t}\right)^{\frac{q_{1}}{p-1}-1}\left(\int_{r}^{\infty}\left(\frac{\mu\left(B_{2 t}(x)\right)}{t^{N-\beta p}}\right)^{\frac{1}{p-1}} \frac{d t}{t}\right)^{\frac{q_{2}}{p-1}}, \\
& \mathbf{D}_{2}(x, r)=r^{\frac{\beta p}{p-1}}\left(\int_{r}^{\infty}\left(\frac{\mu\left(B_{2 t}(x)\right)}{t^{N-\alpha p}}\right)^{\frac{1}{p-1}} \frac{d t}{t}\right)^{\frac{q_{1}}{p-1}}\left(\int_{r}^{\infty}\left(\frac{\mu\left(B_{2 t}(x)\right)}{t^{N-\beta p}}\right)^{\frac{1}{p-1}} \frac{d t}{t}\right)^{\frac{q_{2}}{p-1}-1} .
\end{aligned}
$$

Clearly,

$$
\begin{aligned}
& \int_{r}^{\infty}\left(\frac{\mu\left(B_{2 t}(x)\right)}{t^{N-\beta p}}\right)^{\frac{1}{p-1}} \frac{d t}{t} \leq r^{-\frac{(\alpha-\beta) p}{p-1}} \int_{r}^{\infty}\left(\frac{\mu\left(B_{2 t}(x)\right)}{t^{N-\alpha p}}\right)^{\frac{1}{p-1}} \frac{d t}{t}, \\
& \int_{r}^{\infty}\left(\frac{\mu\left(B_{2 t}(x)\right)}{t^{N-\alpha p}}\right)^{\frac{1}{p-1}} \frac{d t}{t} \leq C r^{\frac{\alpha p}{p-1}-\frac{\alpha p q_{1}+\beta p q_{2}}{(p-1)\left(q_{1}+q_{2}-p+1\right)}} .
\end{aligned}
$$

We derive

$$
\begin{aligned}
\mathbf{D}_{1}(x, r) & \leq C r^{\frac{\alpha p}{p-1}}\left(\int_{r}^{\infty}\left(\frac{\mu\left(B_{2 t}(x)\right)}{t^{N-\alpha p}}\right)^{\frac{1}{p-1}} \frac{d t}{t}\right)^{\frac{q_{1}}{p-1}-1}\left(r^{-\frac{(\alpha-\beta) p}{p-1}} \int_{r}^{\infty}\left(\frac{\mu\left(B_{2 t}(x)\right)}{t^{N-\alpha p}}\right)^{\frac{1}{p-1}} \frac{d t}{t}\right)^{\frac{q_{2}}{p-1}} \\
& =C_{1} r^{\frac{\alpha p}{p-1}-\frac{(\alpha-\beta) p q_{2}}{(p-1)^{2}}}\left(\int_{r}^{\infty}\left(\frac{\mu\left(B_{2 t}(x)\right)}{t^{N-\alpha p}}\right)^{\frac{1}{p-1}} \frac{d t}{t}\right)^{\frac{q_{1}+q_{2}}{p-1}-1} \\
& \leq C_{2} r^{\frac{\alpha p}{p-1}-\frac{(\alpha-\beta) p q_{2}}{(p-1)^{2}}}\left(r^{\frac{\alpha p}{p-1}-\frac{\alpha p q_{1}+\beta p q_{2}}{(p-1)\left(q_{1}+q_{2}-p+1\right)}}\right)^{\frac{q_{1}+q_{2}}{p-1}-1}=C_{3} .
\end{aligned}
$$

Next, we estimate $\mathbf{D}_{2}(x, r)$. If $\frac{q_{1}}{p-1} \geq 1$, similarly as for estimate of $\mathbf{D}_{1}(x, r)$ we obtain $\mathbf{D}_{2}(x, r) \leq C$. If $\frac{q_{1}}{p-1}<1$, we have

$$
\begin{aligned}
& \mathbf{D}_{2}(x, r)=\frac{q_{1}}{p-1} r^{\frac{\beta p}{p-1}} \int_{r}^{\infty}\left(\frac{\mu\left(B_{2 t}(x)\right)}{t^{N-\alpha p}}\right)^{\frac{1}{p-1}}\left(\int_{t}^{\infty}\left(\frac{\mu\left(B_{2 s}(x)\right)}{s^{N-\alpha p}}\right)^{\frac{1}{p-1}} \frac{d s}{s}\right)^{\frac{q_{1}}{p-1}-1} \frac{d t}{t} \times\left(\int_{r}^{\infty}\left(\frac{\mu\left(B_{2 t}(x)\right)}{t^{N-\beta p}}\right)^{\frac{1}{p-1}} \frac{d t}{t}\right)^{\frac{q_{2}}{p-1}-1} \\
& \leq r^{\frac{\beta p}{p-1}} \int_{r}^{\infty}\left(\frac { \mu ( B _ { 2 t } ( x ) ) } { t ^ { N - \alpha p } ) ^ { \frac { 1 } { p - 1 } } } \left(\int _ { t } ^ { \infty } \left(\frac{\mu\left(B_{2 s}(x)\right)}{\left.\left.s^{N-\alpha p}\right)^{\frac{1}{p-1}} \frac{d s}{s}\right)^{\frac{q_{1}}{p-1}-1}}\right.\right.\right. \\
& \times\left(\int_{t}^{\infty}\left(\frac{\mu\left(B_{2 s}(x)\right)}{s^{N-\beta p}}\right)^{\frac{1}{p-1}} \frac{d s}{s}\right)^{\frac{q_{2}}{p-1}-1} \frac{d t}{t}
\end{aligned}
$$


On the other hand,

$$
\begin{aligned}
& \left(\frac{\mu\left(B_{2 t}(x)\right)}{t^{N-\alpha p}}\right)^{\frac{1}{p-1}}=t^{\frac{(\alpha-\beta) p\left(p-1-q_{2}\right)}{(p-1)^{2}}}\left(\left(\frac{\mu\left(B_{2 t}(x)\right)}{t^{N-\alpha p}}\right)^{\frac{1}{p-1}}\right)^{\frac{q_{2}}{p-1}}\left(\left(\frac{\mu\left(B_{2 t}(x)\right)}{t^{N-\beta p}}\right)^{\frac{1}{p-1}}\right)^{1-\frac{q_{2}}{p-1}} \\
& \leq C t^{\frac{(\alpha-\beta) p\left(p-1-q_{2}\right)}{(p-1)^{2}}}\left(\int_{t}^{\infty}\left(\frac{\mu\left(B_{2 s}(x)\right)}{s^{N-\alpha p}}\right)^{\frac{1}{p-1}} \frac{d s}{s}\right)^{\frac{q_{2}}{p-1}}\left(\int_{t}^{\infty}\left(\frac{\mu\left(B_{2 s}(x)\right)}{s^{N-\beta p}}\right)^{\frac{1}{p-1}} \frac{d s}{s}\right)^{1-\frac{q_{2}}{p-1}}
\end{aligned}
$$

therefore,

$$
\begin{aligned}
\mathbf{D}_{2}(x, r) & \leq C r^{\frac{\beta p}{p-1}} \int_{r}^{\infty} t^{\frac{(\alpha-\beta) p\left(p-1-q_{2}\right)}{(p-1)^{2}}}\left(\int_{t}^{\infty}\left(\frac{\mu\left(B_{2 s}(x)\right)}{s^{N-\alpha p}}\right)^{\frac{1}{p-1}} \frac{d s}{s}\right)^{\frac{q_{1}+q_{2}}{p-1}-1} \frac{d t}{t} \\
& \leq C_{1} r^{\frac{\beta p}{p-1}} \int_{r}^{\infty} t^{\frac{(\alpha-\beta) p\left(p-1-q_{2}\right)}{(p-1)^{2}}}\left(r^{\frac{\alpha p}{p-1}-\frac{\alpha p q_{1}+\beta p q_{2}}{(p-1)\left(q_{1}+q_{2}-p+1\right)}}\right)^{\frac{q_{1}+q_{2}}{p-1}-1} \frac{d t}{t} \\
& =C_{2} .
\end{aligned}
$$

Hence,

$$
\int_{0}^{\infty}\left(\frac{\mathbf{A}_{3}(x, r)}{r^{N-\eta p}}\right)^{\frac{1}{p-1}} \frac{d r}{r}+\int_{0}^{\infty}\left(\frac{\mathbf{A}_{4}(x, r)}{r^{N-\eta p}}\right)^{\frac{1}{p-1}} \frac{d r}{r} \leq C \mathbf{W}_{\eta, p}[\mu](x) .
$$

Combining (2.49) with (2.50), (2.51) and (2.52) we obtain

$$
\mathbf{W}_{\eta, p}\left[\left(\mathbf{W}_{\alpha, p}[\mu]\right)^{q_{1}}\left(\mathbf{W}_{\beta, p}[\mu]\right)^{q_{2}}\right] \leq C \mathbf{W}_{\eta, p}[\mu]<\infty,
$$

for $\eta=\alpha$ or $\beta$, provided $\mu$ has compact support in $\mathbb{R}^{N}$. Next, we assume that $\mu$ may not have compact support. Since the above constants noted $C$ are independent of $\mu$, for $n \in \mathbb{N}^{*}$, we set $\mu_{n}=\chi_{B_{n}(0)} \mu$

$$
\mathbf{W}_{\eta, p}\left[\left(\mathbf{W}_{\alpha, p}\left[\mu_{n}\right]\right)^{q_{1}}\left(\mathbf{W}_{\beta, p}\left[\mu_{n}\right]\right)^{q_{2}}\right] \leq C \mathbf{W}_{\eta, p}\left[\mu_{n}\right] \leq C \mathbf{W}_{\eta, p}[\mu]<\infty<C^{\prime},
$$

for $\eta=\alpha$ or $\beta$. Then we infer (e) by Fatou's lemma.

An important step for proving relative compactness in nonlinear problems is the convergence of the nonlinear terms and their equi-integrability is one of the key tool for such a task.

Lemma 2.11 Let $\mu$ be satisfying (2.35) with compact support in $\mathbb{R}^{N}$. Set $\mu_{n}=\varphi_{n} \star \mu$. Then,

$$
\left(\mathbf{W}_{\alpha, p}\left[\mu_{n}\right](x)\right)^{q_{1}}\left(\mathbf{W}_{\beta, p}\left[\mu_{n}\right](x)\right)^{q_{2}}
$$

is equi-integrable in $B_{t}(0)$ for all $t>1$.

Proof. Since supp $\mu_{n} \subset B_{t_{0}}(0)$ for some $t_{0}>0$ and

$$
\left(\mathbf{W}_{\alpha, p}^{2 T}\left[\mu_{n}\right]\right)^{q_{1}} \leq C\left(\mathbf{L}_{\alpha p, \frac{q_{1}}{3 T}\left(q_{1}+q_{2}\right)(p-1)}\left[\mu_{n}\right]\right)^{q_{1}+q_{2}},\left(\mathbf{W}_{\beta, p}^{2 T}\left[\mu_{n}\right]\right)^{q_{2}} \leq C\left(\mathbf{L}_{\beta p, \frac{q_{2}}{3 T}\left(q_{1}+q_{2}\right)(p-1)}\left[\mu_{n}\right]\right)^{q_{1}+q_{2}},
$$

it suffices to show that

$$
\left(\mathbf{L}_{\alpha p, \frac{q_{1}}{\left(q_{1}+q_{2}\right)(p-1)}}^{2\left(t_{0}+t\right)}\left[\mu_{n}\right] \mathbf{L}_{\beta p, \frac{q_{2}}{\left(q_{1}+q_{2}\right)(p-1)}}^{2\left(t_{0}+t\right)}\left[\mu_{n}\right]\right)^{q_{1}+q_{2}}
$$


is equi-integrable in $B_{t}(0)$. Since $\left(\mathbf{I}_{\frac{\alpha p q_{1}+p \beta q_{2}}{q_{1}+q_{2}}}^{2\left(t_{0}+t\right)}\left[\mu_{n}\right]\right)^{\frac{q_{1}+q_{2}}{p-1}} \leq C\left(\mathbf{I}_{\frac{\alpha p q_{1}+p \beta q_{2}}{q_{1}+q_{2}}}^{4\left(t_{0}+t\right)}[\mu] \star \varphi_{n}\right)^{\frac{q_{1}+q_{2}}{p-1}}$, so $\left(\mathbf{I}_{\frac{\alpha p q_{1}+p \beta q_{2}}{q_{1}+q_{2}}}^{2\left(t_{0}+t\right)}\left[\mu_{n}\right]\right)^{\frac{q_{1}+q_{2}}{p-1}}$ is equi-integrable in $B_{t}(0)$ for any $t>t_{0}$. Thus, by [3, Proposition 1.27] we can find a nondecreasing function $\Phi:[0, \infty) \rightarrow[0, \infty)$ such that $\Phi(\lambda) / \lambda \rightarrow \infty$ as $\lambda \rightarrow \infty$, and $\phi\left(2^{j} \lambda\right) \leq j \phi(\lambda)$ for all $\lambda>0, j \in \mathbb{N}$ and $\Phi^{\prime}(\lambda)=\phi(\lambda)$

$$
\int_{0}^{\infty} \phi(\lambda)\left|\left\{\left(\mathbf{I}_{\frac{\alpha p q_{1}+p q_{2}}{q_{1}+q_{2}}}^{2\left(t_{0}+t\right)}\left[\mu_{n}\right]\right)^{\frac{q_{1}+q_{2}}{p-1}}>\lambda\right\}\right| d \lambda \leq 1 .
$$

On the other hand, by Lemma 2.6, there exists $C>0$ and $\varepsilon_{0}>0$ such that

$$
\begin{aligned}
& \left|\left\{\mathbf{L}_{\alpha p, \frac{q_{1}}{\left(q_{1}+q_{2}\right)(p-1)}}^{2\left(t_{0}+t\right)}\left[\mu_{n}\right] \mathbf{L}_{\beta p, \frac{q_{2}}{\left(q_{1}+q_{2}\right)(p-1)}}^{2\left(t_{0}+t\right)}\left[\mu_{n}\right]>a \lambda,\left(\mathbf{I}_{\frac{\alpha p q_{1}+p \beta q_{2}}{q_{1}+q_{2}}}^{2\left(t_{0}+t\right)}\left[\mu_{n}\right]\right)^{\frac{1}{p-1}} \leq \varepsilon \lambda\right\}\right| \\
& \leq C \varepsilon^{\frac{N\left(q_{1}+q_{2}\right)(p-1)}{2 q_{2}(N-\beta p)}}\left|\left\{\mathbf{L}_{\alpha p, \frac{q_{1}}{\left(q_{1}+q_{2}\right)(p-1)}}^{2\left(t_{0}+t\right)}\left[\mu_{n}\right] \mathbf{L}_{\beta p, \frac{q_{2}}{\left(q_{1}+q_{2}\right)(p-1)}}^{2\left(t_{0}+t\right)}\left[\mu_{n}\right]>\varepsilon^{1 / 2} \lambda\right\}\right|
\end{aligned}
$$

for any $\varepsilon \in\left(0, \varepsilon_{0}\right)$ and $t>0$, for some $a>1$. This gives

$$
\begin{aligned}
& \left|\left\{\left(\mathbf{L}_{\alpha p, \frac{q_{1}}{\left(q_{1}+q_{2}\right)(p-1)}}^{2\left(t_{0}+t\right)}\left[\mu_{n}\right] \mathbf{L}_{\beta p, \frac{q_{2}}{\left(q_{1}+q_{2}\right)(p-1)}}^{2\left(t_{0}+t\right)}\left[\mu_{n}\right]\right)^{q_{1}+q_{2}}>a \lambda,\left(\mathbf{I}_{\frac{\alpha p q_{1}+p \beta q_{2}}{q_{1}+q_{2}}}^{2\left(t_{0}+t\right)}\left[\mu_{n}\right]\right)^{\frac{q_{1}+q_{2}}{p-1}} \leq \varepsilon \lambda\right\}\right| \\
& \leq C \varepsilon^{\frac{N(p-1)}{2 q_{2}(N-\beta p)}}\left|\left\{\left(\mathbf{L}_{\alpha p, \frac{q_{1}}{\left(q_{1}+q_{2}\right)(p-1)}}^{2\left(t_{0}+t\right)}\left[\mu_{n}\right] \mathbf{L}_{\beta p, \frac{q_{2}}{\left(q_{1}+q_{2}\right)(p-1)}}^{2\left(t_{0}+t\right)}\left[\mu_{n}\right]\right)^{q_{1}+q_{2}}>\varepsilon^{1 / 2} \lambda\right\}\right|
\end{aligned}
$$

for any $\varepsilon \in\left(0, \varepsilon_{0}\right)$ and $t>0$, for some $a>1$. It is easy to obtain from the above two inequality that

$$
\begin{aligned}
& \int_{0}^{\infty} \phi(\lambda)\left|\left\{\left(\mathbf{L}_{\alpha p, \frac{q_{1}}{\left(q_{1}+q_{2}\right)(p-1)}}^{2\left(t_{0}+t\right)}\left[\mu_{n}\right] \mathbf{L}_{\beta p, \frac{q_{2}}{\left(q_{1}+q_{2}\right)(p-1)}}^{2\left(t_{0}+t\right)}\left[\mu_{n}\right]\right)^{q_{1}+q_{2}}>\lambda\right\}\right| d \lambda \\
& \leq C \varepsilon^{\frac{N(p-1)}{2 q_{2}(N-\beta)}} \int_{0}^{\infty} \phi(\lambda)\left|\left\{\left(\mathbf{L}_{\alpha p, \frac{q_{1}}{\left(q_{1}+q_{2}\right)(p-1)}}^{2\left(t_{0}+\right)_{n}}\left[\mu_{n}\right] \mathbf{L}_{\beta p, \frac{q_{2}}{\left(q_{1}+q_{2}\right)(p-1)}}^{2\left(t_{0}+t\right)}\left[\mu_{n}\right]\right)^{q_{1}+q_{2}}>a^{-1} \varepsilon^{1 / 2} \lambda\right\}\right| d \lambda \\
& +C \int_{0}^{\infty} \phi(\lambda)\left|\left\{\left(\mathbf{I}_{\frac{\alpha p q_{1}+p \beta q_{2}}{q_{1}+q_{2}}}^{2\left(t_{1}+t\right)}\left[\mu_{n}\right]\right)^{\frac{q_{1}+q_{2}}{p-1}}>\varepsilon \lambda\right\}\right| d \lambda \\
& \leq C \varepsilon^{\frac{N(p-1)}{2 q_{2}(N-\beta p)}-1 / 2} \int_{0}^{\infty} \phi\left(a \varepsilon^{-1 / 2} \lambda\right) \mid\left\{\left(\mathbf{L}_{\alpha p, \frac{q_{1}}{\left(q_{1}+q_{2}\right)(p-1)}}^{2\left(t_{0}+t\right)_{n}}\left[\mu_{\beta}\right] \mathbf{L}_{\beta p, \frac{q_{2}}{\left(q_{1}+q_{2}\right)(p-1)}}^{2\left(t_{0}+t\right)_{n}}\left[\mu^{q_{1}+q_{2}}>\lambda\right\} \mid d \lambda\right.\right. \\
& +C \int_{0}^{\infty} \phi(\varepsilon \lambda)\left|\left\{\left(\mathbf{I}_{\frac{\alpha p q_{1}+p \beta q_{2}}{q_{1}+q_{2}}}^{2\left(t_{1}+t\right)}\left[\mu_{n}\right]\right)^{\frac{q_{1}+q_{2}}{p-1}}>\lambda\right\}\right| d \lambda
\end{aligned}
$$

Since $\phi(\varepsilon \lambda), \phi\left(a \varepsilon^{-1 / 2} \lambda\right) \leq C|\log (\varepsilon)| \phi(\lambda)$ for any $\lambda>0, \varepsilon<<1$ and $\frac{N(p-1)}{2 q_{2}(N-\beta p)}-1 / 2>0$, so it is easy to get that

$$
\int_{0}^{\infty} \phi(\lambda)\left|\left\{\left(\mathbf{L}_{\alpha p, \frac{q_{1}}{\left(q_{1}+q_{2}\right)(p-1)}}^{2\left(t_{0}+t\right)}\left[\mu_{n}\right] \mathbf{L}_{\beta p, \frac{q_{2}}{\left(q_{1}+q_{2}\right)(p-1)}}^{2\left(t_{0}+t\right)}\left[\mu_{n}\right]\right)^{q_{1}+q_{2}}>\lambda\right\}\right| d \lambda \leq C
$$


Hence,

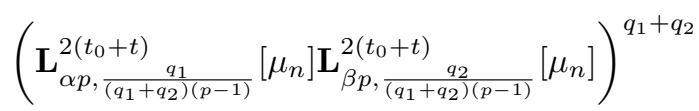

is equi-integrable in $B_{t}(0)$. The proof is complete.

The next statement is the analogue of Theorem 2.10 in a bounded domain.

Theorem 2.12 Let $\alpha, \beta, q_{1}, q_{2}>0, \alpha>\beta, 1<p<\frac{N}{\alpha}, q_{1}+q_{2}>p-1, q_{2}<\frac{N(p-1)}{N-\beta p}$, $\omega \in \mathfrak{M}_{b}^{+}\left(B_{R}\left(x_{0}\right)\right)$ for some $B_{R}\left(x_{0}\right) \subset \mathbb{R}^{N}$, extended by 0 in $B_{R}^{c}\left(x_{0}\right)$. Then, the following statements are equivalent:

(a) The inequality

$$
\omega(K) \leq C_{1} \operatorname{Cap}_{\mathbf{G}_{\frac{\alpha p q_{1}+\beta p q_{2}}{q_{1}+q_{2}}}, \frac{q_{1}+q_{2}}{q_{1}+q_{2}-p+1}}(K),
$$

holds for any compact set $K \subset \mathbb{R}^{N}$, for some $C_{1}=C_{1}(R)>0$.

(b) The inequality

$$
\int_{K}\left(\mathbf{W}_{\alpha, p}^{4 R}[\omega](x)\right)^{q_{1}}\left(\mathbf{W}_{\beta, p}^{4 R}[\omega](x)\right)^{q_{2}} d x \leq C_{2} \operatorname{Cap}_{\mathbf{G}_{\frac{\alpha p q_{1}+\beta p q_{2}}{q_{1}+q_{2}}}, \frac{q_{1}+q_{2}}{q_{1}+q_{2}-p+1}}(K),
$$

holds for any compact set $K \subset \mathbb{R}^{N}$, for some $C_{2}=C_{2}(R)>0$.

(c) The inequality

$$
\int_{\mathbb{R}^{N}}\left(\mathbf{W}_{\frac{\alpha q_{1}+\beta q_{2}}{q_{1}+q_{2}}, p}^{4 R}\left[\chi_{B_{t}(x)} \omega\right](y)\right)^{q_{1}+q_{2}} d y \leq C_{3} \omega\left(B_{t}(x)\right),
$$

holds for any ball $B_{t}(x) \subset \mathbb{R}^{N}$, for some $C_{3}=C_{3}(R)>0$.

(d) The inequality

$$
\int_{\mathbb{R}^{N}}\left(\mathbf{W}_{\alpha, p}^{4 R}\left[\chi_{B_{t}(x)} \omega\right](y)\right)^{q_{1}}\left(\mathbf{W}_{\beta, p}^{4 R}\left[\chi_{B_{t}(x)} \omega\right](y)\right)^{q_{2}} d y \leq C_{4} \omega\left(B_{t}(x)\right),
$$

holds for any ball $B_{t}(x) \subset \mathbb{R}^{N}$, for some $C_{4}=C_{4}(R)>0$.

(e) The system of inequalities

$$
\begin{gathered}
\mathbf{W}_{\alpha, p}^{4 R}\left[\left(\mathbf{W}_{\alpha, p}^{4 R}[\omega]\right)^{q_{1}}\left(\mathbf{W}_{\beta, p}^{4 R}[\omega]\right)^{q_{2}}\right] \leq C_{5} \mathbf{W}_{\alpha, p}^{4 R}[\omega] \\
\mathbf{W}_{\beta, p}^{4 R}\left[\left(\mathbf{W}_{\alpha, p}^{4 R}[\omega]\right)^{q_{1}}\left(\mathbf{W}_{\beta, p}^{4 R}[\omega]\right)^{q_{2}}\right] \leq C_{5} \mathbf{W}_{\beta, p}^{4 R}[\omega],
\end{gathered}
$$

holds in $B_{2 R}\left(x_{0}\right)$ for some $C_{5}=C_{5}(R)>0$.

Proof. By Theorem 2.2 we have (a) $\Leftrightarrow$ (c); by Theorem 2.5, (c) $\Leftrightarrow$ (d). As in the proof of Theorem 2.10, we can see that $(e) \Rightarrow(a)$ and $(e) \Rightarrow(b)$. Since

$$
\begin{aligned}
\left(\mathbf{W}_{\alpha, p}^{4 R}[\omega](x)\right)^{q_{1} \gamma}\left(\mathbf{W}_{\beta, p}^{4 R}[\omega](x)\right)^{q_{2} \gamma} & \geq C \int_{0}^{4 R}\left(\frac{\omega\left(B_{r}(x)\right)}{r^{N-\alpha p}}\right)^{\frac{q_{1} \gamma}{p-1}}\left(\frac{\omega\left(B_{r}(x)\right)}{r^{N-\beta p}}\right)^{\frac{q_{2} \gamma}{p-1}} \frac{d r}{r} \\
& =C \mathbf{W}_{\alpha_{0}, p_{0}}^{4 R}[\omega](x) \quad \text { for all } x \in B_{2 R}\left(x_{0}\right),
\end{aligned}
$$


where $\gamma=\frac{1}{q_{1}}+\frac{1}{q_{2}}, \alpha_{0}=\frac{\gamma\left(\alpha p q_{1}+\beta p q_{2}\right)}{\gamma\left(q_{1}+q_{2}\right)+p-1}$ and $p_{0}=\frac{\gamma\left(q_{1}+q_{2}\right)+p-1}{\gamma\left(q_{1}+q_{2}\right)}$, then, (b) implies that

$$
\int_{K}\left(\mathbf{W}_{\alpha_{0}, p_{0}}^{4 R}[\omega](x)\right)^{\frac{1}{\gamma}} d x \leq C \operatorname{Cap}_{\mathbf{G}_{\frac{\alpha p q_{1}+\beta q_{2}}{q_{1}+q_{2}}}, \frac{q_{1}+q_{2}}{q_{1}+q_{2}-p+1}}(K)=\operatorname{Cap}_{\mathbf{G}_{\alpha_{0} p_{0}, \frac{1}{\gamma}-p_{0}+1}}(K),
$$

is verified for any compact set $K \subset \mathbb{R}^{N}$. Therefore (a) follows by Theorem 2.2.

It remains to prove $(a)+(c)+(d) \Rightarrow(e)$. From (a) we have

$$
\omega\left(B_{r}(x)\right) \leq C r^{N-\frac{\alpha p q_{1}+\beta p q_{2}}{q_{1}+q_{2}-p+1}} \text { for all } x \in \mathbb{R}^{N} \text { and } r>0 .
$$

From (b)

$$
\int_{B_{r}(x)}\left(\mathbf{W}_{\frac{\alpha q_{1}+\beta q_{2}}{q_{1}+q_{2}}, p}^{r}[\omega](y)\right)^{q_{1}+q_{2}} d y \leq C_{2} \omega\left(B_{2 r}(x)\right) \text { for all } x \in \mathbb{R}^{N} \text { and } 0<r<8 R .
$$

Using Hölder's inequality and $\mathbf{W}_{\frac{\alpha q_{1}+\beta q_{2}}{q_{1}+q_{2}}, p}^{r}[\omega] \geq r^{-\frac{(\alpha-\beta) p q_{2}}{(p-1)\left(q_{1}+q_{2}\right)}} \mathbf{W}_{\alpha, p}^{r}[\omega]$, we get

$$
\int_{B_{r}(x)}\left(\mathbf{W}_{\alpha, p}^{r}[\omega](y)\right)^{q_{1}} d y \leq C r^{\frac{(\alpha-\beta) p q_{1} q_{2}+(p-1)\left(q_{2}+(N-\beta p)(p-1) q_{1}\right.}{(p-1)\left(q_{1}+q_{2}\right)}}\left(\frac{\omega\left(B_{2 r}(x)\right)}{r^{N-\beta p}}\right)^{\frac{q_{1}}{q_{1}+q_{2}}}
$$

for all $x \in \mathbb{R}^{N}$ and $0<r<8 R$.

From (c),

$$
\int_{B_{r}(x)}\left(\mathbf{W}_{\alpha, p}^{r}[\omega](y)\right)^{q_{1}}\left(\mathbf{W}_{\beta, p}^{r}[\omega](y)\right)^{q_{2}} d y \leq C_{3} \omega\left(B_{2 r}(x)\right) \text { for all } x \in \mathbb{R}^{N} \text { and } 0<r<8 R \text {. }
$$

By Lemma 2.9,

$$
\int_{B_{r}(x)}\left(\mathbf{W}_{\beta, p}^{r}[\mu]\right)^{q_{2}} d y \leq C r^{N}\left(\frac{\mu\left(B_{2 r}(x)\right)}{r^{N-\beta p}}\right)^{\frac{q_{2}}{p-1}} \text { for all } x \in \mathbb{R}^{N} \text { and } 0<r<8 R .
$$

Next we have for $\eta=\alpha$ or $\eta=\beta$ and almost all $x \in B_{2 R}\left(x_{0}\right)$,

$$
\mathbf{W}_{\eta, p}^{4 R}\left[\left(\mathbf{W}_{\alpha, p}^{4 R}[\mu]\right)^{q_{1}}\left(\mathbf{W}_{\beta, p}^{4 R}[\mu]\right)^{q_{2}}\right](x) \leq C \sum_{i=1}^{4} \int_{0}^{4 R}\left(\frac{\mathbf{A}_{i}(x, r)}{r^{N-\eta p}}\right)^{\frac{1}{p-1}} \frac{d r}{r},
$$

where

$$
\begin{aligned}
& \mathbf{A}_{1}(x, r)=\int_{B_{r}(x)}\left(\mathbf{W}_{\alpha, p}^{r}[\mu](y)\right)^{q_{1}}\left(\mathbf{W}_{\beta, p}^{r}[\mu](y)\right)^{q_{2}} d y, \\
& \mathbf{A}_{2}(x, r)=\int_{B_{r}(x)}\left(\mathbf{W}_{\alpha, p}^{r}[\mu](y)\right)^{q_{1}}\left(\int_{r}^{4 R}\left(\frac{\mu\left(B_{t}(y)\right)}{t^{N-\beta p}}\right)^{\frac{1}{p-1}} \frac{d t}{t}\right)^{q_{2}} d y, \\
& \mathbf{A}_{3}(x, r)=\int_{B_{r}(x)}\left(\int_{r}^{4 R}\left(\frac{\mu\left(B_{t}(y)\right)}{t^{N-\alpha p}}\right)^{\frac{1}{p-1}} \frac{d t}{t}\right)^{q_{1}}\left(\mathbf{W}_{\beta, p}^{r}[\mu](y)\right)^{q_{2}} d y, \\
& \mathbf{A}_{4}(x, r)=\int_{B_{r}(x)}\left(\int_{r}^{4 R}\left(\frac{\mu\left(B_{t}(y)\right)}{t^{N-\alpha p}}\right)^{\frac{1}{p-1}} \frac{d t}{t}\right)^{q_{1}}\left(\int_{r}^{4 R}\left(\frac{\mu\left(B_{t}(y)\right)}{t^{N-\beta p}}\right)^{\frac{1}{p-1}} \frac{d t}{t}\right)^{q_{2}} d y .
\end{aligned}
$$


Thanks to (2.47) there holds

$$
\mathbf{A}_{1}(x, r) \leq C \mu\left(B_{2 r}(x)\right)
$$

which implies

$$
\int_{0}^{4 R}\left(\frac{\mathbf{A}_{1}(x, r)}{r^{N-\eta p}}\right)^{\frac{1}{p-1}} \frac{d r}{r} \leq C \mathbf{W}_{\eta, p}^{8 R}[\mu](x) \leq C \mathbf{W}_{\eta, p}^{4 R}[\mu](x) \text { for all } x \in B_{2 R}\left(x_{0}\right) .
$$

Since $B_{t}(y) \leq B_{2 t}(x)$ for any $y \in B_{r}(x)$ and $t \geq r$, and thanks to (2.46) and (2.9) we deduce that there holds, for $0<r<4 R$ and $x \in B_{2 R}\left(x_{0}\right)$,

$$
\begin{aligned}
\mathbf{A}_{2}(x, r) & \leq \int_{B_{r}(x)}\left(\mathbf{W}_{\alpha, p}^{r}[\mu](y)\right)^{q_{1}} d y\left(\int_{r}^{4 R}\left(\frac{\mu\left(B_{2 t}(x)\right)}{t^{N-\beta p}}\right)^{\frac{1}{p-1}} \frac{d t}{t}\right)^{q_{2}} \\
& \leq C r^{\frac{(\alpha-\beta) p q_{1} q_{2}+(p-1) N q_{2}+(N-\beta p)(p-1) q_{1}}{(p-1)\left(q_{1}+q_{2}\right)}}\left(\frac{\mu\left(B_{2 r}(x)\right)}{r^{N-\beta p}}\right)^{\frac{q_{1}}{q_{1}+q_{2}}}\left(\int_{r}^{4 R}\left(\frac{\mu\left(B_{2 t}(x)\right)}{t^{N-\beta p}}\right)^{\frac{1}{p-1}} \frac{d t}{t}\right)^{q_{2}} \\
& \leq C r^{\frac{(\alpha-\beta) p q_{1} q_{2}+(p-1) N q_{2}+(N-\beta p)(p-1) q_{1}}{(p-1)\left(q_{1}+q_{2}\right)}}\left(\int_{r}^{4 R}\left(\frac{\mu\left(B_{2 t}(x)\right)}{t^{N-\beta p}}\right)^{\frac{1}{p-1}} \frac{d t}{t}\right)^{q_{2}+\frac{q_{1}(p-1)}{q_{1}+q_{2}}}
\end{aligned}
$$

Next

$$
\begin{aligned}
\mathbf{A}_{3}(x, r) & \leq\left(\int_{r}^{4 R}\left(\frac{\mu\left(B_{2 t}(x)\right)}{t^{N-\alpha p}}\right)^{\frac{1}{p-1}} \frac{d t}{t}\right)^{q_{1}} \int_{B_{r}(x)}\left(\mathbf{W}_{\beta, p}^{r}[\mu](y)\right)^{q_{2}} d y \\
& \leq C\left(\int_{r}^{4 R}\left(\frac{\mu\left(B_{2 t}(x)\right)}{t^{N-\alpha p}}\right)^{\frac{1}{p-1}} \frac{d t}{t}\right)^{q_{1}} r^{N}\left(\frac{\mu\left(B_{2 r}(x)\right)}{r^{N-\beta p}}\right)^{\frac{q_{2}}{p-1}} \\
& \leq C r^{N}\left(\int_{r}^{4 R}\left(\frac{\mu\left(B_{2 t}(x)\right)}{t^{N-\alpha p}}\right)^{\frac{1}{p-1}} \frac{d t}{t}\right)^{q_{1}}\left(\int_{r}^{4 R}\left(\frac{\mu\left(B_{2 t}(x)\right)}{t^{N-\beta p}}\right)^{\frac{1}{p-1}} \frac{d t}{t}\right)^{q_{2}},
\end{aligned}
$$

and

$$
\mathbf{A}_{4}(x, r) \leq C r^{N}\left(\int_{r}^{4 R}\left(\frac{\mu\left(B_{2 t}(x)\right)}{t^{N-\alpha p}}\right)^{\frac{1}{p-1}} \frac{d t}{t}\right)^{q_{1}}\left(\int_{r}^{4 R}\left(\frac{\mu\left(B_{2 t}(x)\right)}{t^{N-\beta p}}\right)^{\frac{1}{p-1}} \frac{d t}{t}\right)^{q_{2}} .
$$

As in the proof of Theorem 2.10, we easily obtain

$$
\int_{0}^{4 R}\left(\frac{\mathbf{A}_{2}(x, r)}{r^{N-\eta p}}\right)^{\frac{1}{p-1}} \frac{d r}{r} \leq C \mathbf{W}_{\eta, p}^{4 R}[\mu](x) \text { for all } x \in B_{2 R}\left(x_{0}\right)
$$

and

$$
\int_{0}^{4 R}\left(\frac{\mathbf{A}_{3}(x, r)}{r^{N-\eta p}}\right)^{\frac{1}{p-1}} \frac{d r}{r}+\int_{0}^{4 R}\left(\frac{\mathbf{A}_{4}(x, r)}{r^{N-\eta p}}\right)^{\frac{1}{p-1}} \frac{d r}{r} \leq C \mathbf{W}_{\eta, p}^{4 R}[\mu](x) \text { for all } x \in B_{2 R}\left(x_{0}\right) .
$$

Combining these inequalities with (2.66) and (2.67), we get (e). 


\section{Renormalized solutions}

Let $\Omega$ be a bounded domain in $\mathbb{R}^{N}$. If $\mu \in \mathfrak{M}_{b}(\Omega)$, we denote by $\mu^{+}$and $\mu^{-}$respectively its positive and negative parts in the Jordan decomposition. We denote by $\mathfrak{M}_{0}(\Omega)$ the space of diffuse measures in $\Omega$ and by $\mathfrak{M}_{s}(\Omega)$ the space of measures in $\Omega$ which are singular with respect to the $\operatorname{Cap}_{\mathbf{G}_{1}, p}$ which means that their support is set of zero $\operatorname{Cap}_{\mathbf{G}_{1}, p}$-capacity. Classically, any $\mu \in \mathfrak{M}_{b}(\Omega)$ can be written in a unique way under the form $\mu=\mu_{0}+\mu_{s}$ where $\mu_{0} \in \mathfrak{M}_{0}(\Omega) \cap \mathfrak{M}_{b}(\Omega)$ and $\mu_{s} \in \mathfrak{M}_{s}(\Omega)$. It is well known that any $\mu_{0} \in \mathfrak{M}_{0}(\Omega) \cap \mathfrak{M}_{b}(\Omega)$ can be written under the form $\mu_{0}=f-\operatorname{div} g$ where $f \in L^{1}(\Omega)$ and $g \in L^{p^{\prime}}\left(\Omega, \mathbb{R}^{N}\right)$.

For $k>0$ and $s \in \mathbb{R}$ we set $T_{k}(s)=\max \{\min \{s, k\},-k\}$. If $u$ is a measurable function defined in $\Omega$, finite a.e. and such that $T_{k}(u) \in W_{l o c}^{1, p}(\Omega)$ for any $k>0$, there exists a measurable function $v: \Omega \mapsto \mathbb{R}^{N}$ such that $\nabla T_{k}(u)=\chi_{|u| \leq k} v$ a.e. in $\Omega$ and for all $k>0$. We define the gradient of $u$ by $v=\nabla u$ almost everywhere. We recall the definition of a renormalized solution given in [9].

Definition 3.1 Let $A: \mathbb{R}^{N} \mapsto \mathbb{R}^{N}$ satisfy (1.2). Let $\mu=\mu_{0}+\mu_{s} \in \mathfrak{M}_{b}(\Omega)$. A measurable function $u$ defined in $\Omega$ and finite a.e. is called a renormalized solution of

$$
\begin{aligned}
-\operatorname{div}(A(x, \nabla u)) & =\mu & & \text { in } \Omega, \\
u & =0 & & \text { on } \partial \Omega,
\end{aligned}
$$

if $T_{k}(u) \in W_{0}^{1, p}(\Omega)$ for any $k>0,|\nabla u|^{p-1} \in L^{r}(\Omega)$ for any $0<r<\frac{N}{N-1}$, and $u$ has the property that for any $k>0$ there exist $\lambda_{k}^{+}$and $\lambda_{k}^{-}$belonging to $\mathfrak{M}_{b}^{+} \cap \mathfrak{M}_{0}(\Omega)$, respectively concentrated on the sets $u=k$ and $u=-k$, with the property that $\mu_{k}^{+} \rightarrow \mu_{s}^{+}, \mu_{k}^{-} \rightarrow \lambda_{s}^{-}$in the narrow topology of measures and such that

$$
\int_{\{|u|<k\}} A(x, \nabla u) \cdot \nabla \varphi d x=\int_{\{|u|<k\}} \varphi d \mu_{0}+\int_{\Omega} \varphi d \lambda_{k}^{+}-\int_{\Omega} \varphi d \lambda_{k}^{-},
$$

for every $\varphi \in W_{0}^{1, p}(\Omega) \cap L^{\infty}(\Omega)$.

Proposition 3.2 [26] If $\mu \in \mathfrak{M}_{0}(\Omega)$, then problem (3.1) has a unique renormalized solution.

We recall the next two important results which are proved in [9, Th 4.1, Sec 5.1].

Theorem 3.3 Let $\left\{\mu_{n}\right\} \subset \mathfrak{M}_{b}(\Omega)$ be a sequence such that $\sup _{n}\left|\mu_{n}\right|(\Omega)<\infty$ and let $\left\{u_{n}\right\}$ be renormalized solutions of

$$
\begin{aligned}
\left.-\operatorname{div} A\left(x, \nabla u_{n}\right)\right) & =\mu_{n} & & \text { in } \Omega \\
u_{n} & =0 & & \text { on } \partial \Omega .
\end{aligned}
$$

Then, up to a subsequence, $\left\{u_{n}\right\}$ converges a.e. to a solution $u$ of $-\operatorname{div}(A(x, \nabla u))=\mu$ in the sense of distributions in $\Omega$, for some measure $\mu \in \mathfrak{M}_{b}(\Omega)$, and for every $k>0$, $k^{-1}\left\|\nabla T_{k}(u)\right\|_{L^{p}}^{p} \leq M$ for some $M>0$.

The following fundamental stability result of [9] extends Theorem 3.3.

Theorem 3.4 Let $\mu=\mu_{0}+\mu_{s}^{+}-\mu_{s}^{-} \in \mathfrak{M}_{b}(\Omega)$, with $\mu_{0}=f-\operatorname{divg} \in \mathfrak{M}_{0}(\Omega)$, $\mu_{s}^{+}, \mu_{s}^{-} \in$ $\mathfrak{M}_{s}^{+}(\Omega)$. Assume there are sequences $\left\{f_{n}\right\} \subset L^{1}(\Omega),\left\{g_{n}\right\}, \subset\left(L^{p^{\prime}}(\Omega)\right)^{N},\left\{\eta_{n}^{1}\right\},\left\{\eta_{n}^{2}\right\} \subset$ $\mathfrak{M}_{b}^{+}(\Omega)$ such that $f_{n} \rightarrow f$ weakly in $L^{1}(\Omega), g_{n} \rightarrow g$ in $L^{p^{\prime}}(\Omega)$ and div $g_{n}$ is bounded in $\mathfrak{M}_{b}(\Omega), \eta_{n}^{1} \rightarrow \mu_{s}^{+}$and $\eta_{n}^{2} \rightarrow \mu_{s}^{-}$in the narrow topology. If $\mu_{n}=f_{n}-\operatorname{div} g_{n}+\eta_{n}^{1}-\eta_{n}^{2}$ and $u_{n}$ is a renormalized solution of (3.2), then, up to a subsequence, $u_{n}$ converges a.e. to a renormalized solution $u$ of $(3.1)$. Furthermore, $T_{k}\left(u_{n}\right) \rightarrow T_{k}(u)$ in $W_{0}^{1, p}(\Omega)$ for any $k>0$. 
The next result is proved in [6, Th 3.2]. Therein it plays an important role in study the stability of the renormalized solutions of the following problem with absorption,

$$
\begin{aligned}
-\operatorname{div}\left(A\left(x, \nabla u_{n_{k}}\right)\right)+\left|u_{n_{k}}\right|^{q-1} u_{n_{k}}=\mu_{n_{k}} & \text { in } \Omega, \\
u_{n_{k}}=0 & \text { on } \partial \Omega .
\end{aligned}
$$

Theorem 3.5 Let $\left\{n_{k}\right\}_{k}$ be an increasing sequence in $\mathbb{N}, q>p-1,\left\{\mu_{n_{k}}\right\}_{k}$ be a sequence in $\mathfrak{M}\left(\mathbb{R}^{N}\right)$ such that

$$
\sup _{k \geq k_{0}}\left|\mu_{n_{k}}\right|\left(B_{n_{k_{0}}}(0)\right)<+\infty \text { for all } k_{0} \in \mathbb{N} .
$$

Let $u_{n_{k}}$ be a renormalized solution of (3.1) with data $\mu_{n_{k}}$ and $\Omega=B_{n_{k}}(0)$ such that $\left\{\left|u_{n_{k}}\right|^{q}\right\}_{k \geq k_{0}}$ is bounded in $L^{1}\left(B_{n_{k_{0}}}(0)\right)$ for any $k_{0}$. Then, there exist subsequence of $\left\{u_{n_{k}}\right\}_{k}$, still denoted by $\left\{u_{n_{k}}\right\}_{k}$ a measure $\mu$ and measurable function $u$ such that $\mu_{n_{k}} \rightarrow \mu$ in the weak sense of measures, $u_{n_{k}} \rightarrow u, \nabla u_{n_{k}} \rightarrow \nabla u$ a.e in $\mathbb{R}^{N}$. Moreover, $\left|\nabla u_{n_{k}}\right|^{p-2} \nabla u_{n_{k}} \rightarrow$ $|\nabla u|^{p-2} \nabla u$ in $L_{\text {loc }}^{s}\left(\mathbb{R}^{N}\right)$ for all $0 \leq s<\frac{N}{N-1}$ and $u$ satisfies (3.1) in the sense of distributions in $\mathbb{R}^{N}$.

Theorem 3.6 $[22,7]$ Let $\Omega$ be a bounded domain of $\mathbb{R}^{N}$. Then there exists a constant $C=C\left(N, p, \Lambda_{1}, \Lambda_{2}\right)>1$ such that if $\mu \in \mathfrak{M}_{b}(\Omega)$ and $u$ is a renormalized solution of problem (3.1) there holds

$$
|u(x)| \leq C \mathbf{W}_{1, p}^{2 R}[|\mu|](x) \quad \text { a.e. in } \Omega,
$$

where $R=\operatorname{diam}(\Omega)$. Moreover, if $\mu \geq 0$ and $u \geq 0$ then,

$$
u(x) \geq \frac{1}{C} \mathbf{W}_{1, p}^{\frac{d(x, \partial \Omega)}{4}}[\mu](x) \text { a.e in } \Omega .
$$

Theorem 3.7 $[10,14,17]$ Let $\Omega$ be a bounded domain of $\mathbb{R}^{N}$. Then there exists a constant $C=C\left(N, p, \Lambda_{1}, \Lambda_{2}\right.$, diam $\left.(\Omega)\right)>0$ such that if $\mu \in C_{b}(\Omega)$ and $u$ is a solution of problem (3.1) there holds

$$
|\nabla u(x)| \leq C\left(\mathbf{I}_{1}^{r}[|\mu|](x)\right)^{\frac{1}{p-1}}+C\left(f_{B_{r}(x)}|\nabla u|^{\gamma_{0}} d x\right)^{1 / \gamma_{0}}
$$

for any $B_{r}(x) \subseteq \Omega$ and for some $\gamma_{0} \in\left(0, \frac{N(p-1)}{N-1}\right)$. Moreover, if $A(x, \xi)=A(\xi)$ for any $(x, \xi) \in \mathbb{R}^{N} \times \mathbb{R}^{N}$, then the constant in (3.6) does not depend on diam $(\Omega)$.

Corollary 3.8 Let $\Omega$ be a bounded domain of $\mathbb{R}^{N}, R=\operatorname{diam}(\Omega), \mu \in \mathfrak{M}_{b}(\Omega)$. Then there exists a constant $C=C\left(N, p, \Lambda_{1}, \Lambda_{2}\right)>0$ and a renormalized solution $u$ of problem (3.1) such that

$$
|\nabla u(x)| \leq C\left(\frac{R}{\delta}\right)^{N}\left(\mathbf{I}_{1}^{2 R}[|\mu|](x)\right)^{\frac{1}{p-1}},
$$

for any $x \in \Omega$ such that $d(x, \partial \Omega)>\delta$ with $\delta \in(0, R / 2)$. Moreover, if $A(x, \xi)=A(\xi)$ for any $(x, \xi) \in \mathbb{R}^{N} \times \mathbb{R}^{N}$, then the constant in (3.7) does not depend on $R$.

Proof. We can choose $\mu_{n} \in C_{c}^{\infty}(\Omega)$ such that $\mu_{n}$ converges to $\mu$ in the sense of theorem 3.4 and $\left|\mu_{n}\right| \leq \varphi_{n} *|\mu|$, where $\left\{\varphi_{n}\right\}$ is a sequence of mollifiers in $\mathbb{R}^{N}$. Let $u_{n}$ be solutions of problem (3.1) with data $\mu_{n}$. Fixed $\delta \in(0, R / 2)$, by Theorem 3.7 , we have

$$
\left|\nabla u_{n}(x)\right| \leq C\left(\mathbf{I}_{1}^{\delta / 2}\left[\left|\mu_{n}\right|\right](x)\right)^{\frac{1}{p-1}}+C f_{B_{\delta / 2}(x)}\left|\nabla u_{n}\right| d x,
$$


for any $x \in \Omega, d(x, \partial \Omega)>\delta$. Notice that (see e.g. [9])

$$
\left|\left\{\left|\nabla u_{n}\right|>s\right\}\right| \leq C \frac{\left(\left|\mu_{n}\right|(\Omega)\right)^{\frac{N}{N-1}}}{s^{\frac{N(N-1)}{N-1}}} \text { for all } s>0 .
$$

It leads to

$$
\int_{\Omega}\left|\nabla u_{n}(x)\right| d x \leq C R^{N}\left(\frac{\left|\mu_{n}\right|(\Omega)}{R^{N-1}}\right)^{\frac{1}{p-1}} .
$$

Thus,

$$
\begin{aligned}
\left|\nabla u_{n}(x)\right| & \leq C\left(\frac{R}{\delta}\right)^{N}\left(\mathbf{I}_{1}^{2 R}\left[\left|\mu_{n}\right|\right](x)\right)^{\frac{1}{p-1}} \\
& \leq C\left(\frac{R}{\delta}\right)^{N}\left(\left(\varphi_{n} * \mathbf{I}_{1}^{2 R}[|\mu|]\right)(x)\right)^{\frac{1}{p-1}}
\end{aligned}
$$

for any $x \in \Omega, d(x, \partial \Omega)>\delta$.

On the other hand, by theorem 3.4, there exists a subsequence of $\left\{u_{n}\right\}$ converging to a renormalized solution $u$ of (3.1) with data $\mu_{n}$. Therefore, $u$ satisfies (3.7) since $\varphi_{n} * \mathbf{I}_{1}^{2 R}[|\mu|] \rightarrow$ $\mathbf{I}_{1}^{2 R}[|\mu|]$ almost everywhere.

\section{Proof of the main results}

Proof of Theorem 1.1.

Step 1: Case $\frac{3 N-2}{2 N-1}<p \leq 2$. Let $\mu_{n, k} \in C_{c}^{\infty}\left(B_{2 k}(0)\right)$ for $k \in \mathbb{N}$ such that $\mu_{n, k}$ converges to $\chi_{B_{k}(0)} \mu$ in the sense of theorem 3.4 with $\Omega=B_{2 k}(0)$ and $\left|\mu_{n, k}\right| \leq \varphi_{n} *\left(\chi_{B_{k}(0)}|\mu|\right)$, where $\left\{\varphi_{n}\right\}$ is a sequence of mollifiers in $\mathbb{R}^{N}$. Thanks to Proposition 2.3,

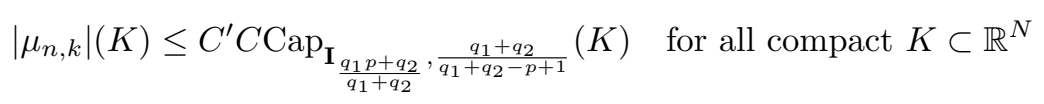

We will prove that if $C$ in (4.1) is small enough, then for any $k \geq 1, n \in \mathbb{N}$ the problem

$$
\begin{aligned}
-\operatorname{div}\left(A\left(\nabla u_{n, k}\right)\right) & =\chi_{B_{k}(0)}\left|u_{n, k}\right|^{q_{1}-1} u\left|\nabla u_{n, k}\right|^{q_{2}}+\mu_{n, k} & & \text { in } B_{2 k}(0), \\
u_{n, k} & =0 & & \text { on } \partial B_{2 k}(0),
\end{aligned}
$$

has a renormalized solution satisfying

$$
\left|u_{n, k}\right| \leq C_{0}\left(\mathbf{I}_{p}\left[\left|\mu_{n, k}\right|\right]\right)^{\frac{1}{p-1}}, \quad\left|\nabla u_{n, k}\right| \leq C_{0}\left(\mathbf{I}_{1}\left[\left|\mu_{n, k}\right|\right]\right)^{\frac{1}{p-1}} \quad \text { in } B_{k}(0) .
$$

By Theorem (2.10), we need to prove that there exists $M>0$ such that, if for $\alpha=1$ and $p$, the following inequalities hold,

$$
\mathbf{I}_{\alpha}\left[\left(\mathbf{I}_{p}\left[\left|\mu_{n, k}\right|\right]\right)^{\frac{q_{1}}{p-1}}\left(\mathbf{I}_{1}\left[\left|\mu_{n, k}\right|\right]\right)^{\frac{q_{2}}{p-1}}\right] \leq M \mathbf{I}_{\alpha}\left[\left|\mu_{n, k}\right|\right]<\infty \quad \text { almost everywhere, }
$$

then problem (4.2) has a renormalized solution satisfying (4.3).

For any $k \in \mathbb{N}$, we set

$$
\mathbf{E}_{\Lambda}=\left\{u:|u| \leq \Lambda\left(\mathbf{I}_{p}\left[\left|\mu_{n, k}\right|\right]\right)^{\frac{1}{p-1}}, \quad|\nabla u| \leq \Lambda\left(\mathbf{I}_{1}\left[\left|\mu_{n, k}\right|\right]\right)^{\frac{1}{p-1}} \text { in } B_{k}(0)\right\} .
$$


Since $\mu_{n, k} \in C_{c}^{\infty}\left(B_{2 k}(0)\right), \mathbf{E}_{\Lambda} \subset W_{0}^{1, \infty}\left(B_{2 k}(0)\right)$. Clearly, $\mathbf{E}_{\Lambda}$ is convex and closed under the strong topology of $W_{0}^{1,1}\left(B_{2 k}(0)\right)$. Moreover, if $u \in \mathbf{E}_{\Lambda}$, then $|u|^{q_{1}}|\nabla u|^{q_{2}} \in L^{1}\left(B_{k}(0)\right)$.

We consider the map $S: \mathbf{E}_{\Lambda} \mapsto W_{0}^{1,1}(\Omega)$ defined for each $v \in \mathbf{E}_{\Lambda}$ by $S(v)=u$, where $u \in W_{0}^{1,1}(\Omega)$ is the unique renormalized solution of

$$
\begin{array}{cl}
-\operatorname{div}(A(\nabla u))=\chi_{B_{k}(0)}|v|^{q_{1}-1} v|\nabla v|^{q_{2}}+\mu_{n, k} & \text { in } B_{2 k}(0), \\
u=0 & \text { on } \partial B_{2 k}(0) .
\end{array}
$$

Notice that $\mathbf{W}_{1, p}\left[\left|\mu_{n, k}\right|\right] \leq C\left(\mathbf{I}_{p}\left[\left|\mu_{n, k}\right|\right]\right)^{\frac{1}{p-1}}$, this is due to the fact that, since $1<p<2$, then

$$
W_{1, p}[\mu](x) \sim \sum a_{n}^{\frac{1}{p-1}} \leq\left(\sum a_{n}\right)^{\frac{1}{p-1}} \sim\left(I_{p}[\mu]\right)^{1 /(p-1)} \text { with } a_{n}=\frac{\mu\left(B\left(x, 2^{n}\right)\right)}{2^{n(N-p)}}
$$

By Theorem 3.6 and Corollary 3.8, we have

$$
\begin{aligned}
& |u| \leq C\left(\mathbf{I}_{p}\left[\chi_{B_{k}(0)}|v|^{q_{1}}|\nabla v|^{q_{2}} d x+\left|\mu_{n, k}\right|\right]\right)^{\frac{1}{p-1}}, \\
& |\nabla u| \leq C\left(\mathbf{I}_{1}\left[\chi_{B_{k}(0)}|v|^{q_{1}}|\nabla v|^{q_{2}} d x+\left|\mu_{n, k}\right|\right]\right)^{\frac{1}{p-1}},
\end{aligned}
$$

in $B_{k}(0)$. By the definition of $v$ we get

$$
\begin{aligned}
& |u| \leq C\left(\Lambda^{q_{1}+q_{2}} \mathbf{I}_{p}\left[\left(\mathbf{I}_{p}\left[\left|\mu_{n, k}\right|\right]\right)^{\frac{q_{1}}{p-1}}\left(\mathbf{I}_{1}\left[\left|\mu_{n, k}\right|\right]\right)^{\frac{q_{2}}{p-1}}\right]+\mathbf{I}_{p}\left[\left|\mu_{n, k}\right|\right]\right)^{\frac{1}{p-1}}, \\
& |\nabla u| \leq C\left(\Lambda^{q_{1}+q_{2}} \mathbf{I}_{1}\left[\left(\mathbf{I}_{p}\left[\left|\mu_{n, k}\right|\right]\right)^{\frac{q_{1}}{p-1}}\left(\mathbf{I}_{1}\left[\left|\mu_{n, k}\right|\right]\right)^{\frac{q_{2}}{p-1}}\right]+\mathbf{I}_{1}\left[\left|\mu_{n, k}\right|\right]\right)^{\frac{1}{p-1}},
\end{aligned}
$$

in $B_{k}(0)$. Using (4.4), we obtain

$$
\begin{aligned}
& |u| \leq C\left(\Lambda^{q_{1}+q_{2}} M \mathbf{I}_{p}\left[\left|\mu_{n, k}\right|\right]+\mathbf{I}_{p}\left[\left|\mu_{n, k}\right|\right]\right)^{\frac{1}{p-1}}=C\left(\Lambda^{q_{1}+q_{2}} M+1\right)^{\frac{1}{p-1}}\left(\mathbf{I}_{p}\left[\left|\mu_{n, k}\right|\right]\right)^{\frac{1}{p-1}}, \\
& |\nabla u| \leq C\left(\Lambda^{q_{1}+q_{2}} M \mathbf{I}_{1}\left[\left|\mu_{n, k}\right|\right]+\mathbf{I}_{1}\left[\left|\mu_{n, k}\right|\right]\right)^{\frac{1}{p-1}}=C\left(\Lambda^{q_{1}+q_{2}} M+1\right)^{\frac{1}{p-1}}\left(\mathbf{I}_{1}\left[\left|\mu_{n, k}\right|\right]\right)^{\frac{1}{p-1}},
\end{aligned}
$$

in $B_{k}(0)$. We choose

$$
\Lambda=C\left(\frac{q_{1}+q_{2}}{q_{1}+q_{2}-p+1}\right)^{\frac{1}{p-1}}, \quad M=\Lambda^{-q_{1}-q_{2}}\left(\left(\frac{q_{1}+q_{2}}{q_{1}+q_{2}-p+1}\right)^{\frac{1}{p-1}}-1\right)
$$

then $C\left(\Lambda^{q_{1}+q_{2}} M+1\right)^{\frac{1}{p-1}}=\Lambda$ and $u \in \mathbf{E}_{\Lambda}$. Hence, $S\left(\mathbf{E}_{\Lambda}\right) \subset \mathbf{E}_{\Lambda}$.

Next we show that $S: \mathbf{E}_{\Lambda} \mapsto \mathbf{E}_{\Lambda}$ is continuous. Let $\left\{v_{n}\right\}$ be a sequence in $\mathbf{E}_{\Lambda}$ such that $v_{m}$ converges strongly in $W_{0}^{1,1}\left(B_{2 k}(0)\right)$ to a function $v \in \mathbf{E}_{\Lambda}$. Set $u_{m}=S\left(v_{m}\right)$. We need to show that $u_{m} \rightarrow S(v)$ in $W_{0}^{1,1}\left(B_{2 k}(0)\right)$. We have

$$
\begin{aligned}
-\operatorname{div}\left(A\left(\nabla u_{m}\right)\right) & =\chi_{B_{k}(0)}\left|v_{m}\right|^{q_{1}-1} v_{m}\left|\nabla v_{m}\right|^{q_{2}}+\mu_{n, k} & & \text { in } B_{2 k}(0), \\
u_{m} & =0 & & \text { on } \partial B_{2 k}(0),
\end{aligned}
$$

and

$$
\left|u_{m}\right|,\left|v_{m}\right| \leq \Lambda\left(\mathbf{I}_{p}\left[\left|\mu_{n, k}\right|\right]\right)^{\frac{1}{p-1}}, \quad\left|\nabla u_{m}\right|,\left|\nabla v_{m}\right| \leq \Lambda\left(\mathbf{I}_{1}\left[\left|\mu_{n, k}\right|\right]\right)^{\frac{1}{p-1}} \quad \text { in } B_{k}(0) .
$$

Since $\left(\mathbf{I}_{p}\left[\left|\mu_{n, k}\right|\right]\right)^{\frac{q_{1}}{p-1}}\left(\mathbf{I}_{1}\left[\left|\mu_{n, k}\right|\right]\right)^{\frac{q_{2}}{p-1}} \in L_{l o c}^{1}\left(\mathbb{R}^{N}\right)$, we obtain 


$$
\chi_{B_{k}(0)}\left|v_{m}\right|^{q_{1}-1} v_{m}\left|\nabla v_{m}\right|^{q_{2}} \rightarrow \chi_{B_{k}(0)}|v|^{q_{1}-1} v|\nabla v|^{q_{2}} \quad \text { as } n \rightarrow \infty .
$$

Applying Theorem 3.4, we derive that $u_{n} \rightarrow S(v)$ in $W_{0}^{1,1}\left(B_{2 k}(0)\right)$ as $n \rightarrow \infty$. Similarly, we can prove that $S\left(\mathbf{E}_{\Lambda}\right)$ is pre-compact under the strong topology of $W_{0}^{1,1}\left(B_{2 k}(0)\right)$.

Thus, by Schauder Fixed Point Theorem, $S$ has a fixed point on $\mathbf{E}_{\Lambda}$. This means that for any $k, n \in \mathbb{N}$, problem (4.2) has a renormalized solution $u_{n, k}$ satisfying (4.3).

By Lemma 2.11, $\left\{\left(\mathbf{I}_{p}\left[\left|\mu_{n, k}\right|\right]\right)^{\frac{q_{1}}{p-1}}\left(\mathbf{I}_{1}\left[\left|\mu_{n, k}\right|\right]\right)^{\frac{q_{2}}{p-1}}\right\}_{n}$ is equi-integrable in $B_{2 k}(0)$. Thus, by a standard compactness argument, we get that $u_{n, k}$ converges to a renormalized solution $u_{k}$ of

$$
\begin{aligned}
-\operatorname{div}\left(A\left(\nabla u_{k}\right)\right) & =\chi_{B_{k}(0)}\left|u_{k}\right|^{q_{1}-1} u_{k}\left|\nabla u_{k}\right|^{q_{2}}+\chi_{B_{k}(0)} \mu & & \text { in } B_{2 k}(0), \\
u_{k} & =0 & & \text { on } \partial B_{2 k}(0),
\end{aligned}
$$

which satisfies

$$
\left|u_{k}\right| \leq C_{0}\left(\mathbf{I}_{p}[|\mu|]\right)^{\frac{1}{p-1}}, \quad\left|\nabla u_{k}\right| \leq C_{0}\left(\mathbf{I}_{1}[|\mu|]\right)^{\frac{1}{p-1}} \quad \text { in } B_{k}(0) .
$$

Finally, thanks to Theorem 3.5, there exists a subsequence of $\left\{u_{k}\right\}_{k}$, still denoted by $\left\{u_{k}\right\}_{k}$ and $u \in W_{l o c}^{1,1}\left(\mathbb{R}^{N}\right)$ such that $u_{k}$ converges to $u$ and $\nabla u_{k}$ converges to $\nabla u$ almost everywhere. Since

$$
\chi_{B_{k}(0)}\left|u_{k}\right| \leq C_{0}\left(\mathbf{I}_{p}[|\mu|]\right)^{\frac{1}{p-1}}, \quad \chi_{B_{k}(0)}\left|\nabla u_{k}\right| \leq C_{0}\left(\mathbf{I}_{1}[|\mu|]\right)^{\frac{1}{p-1}} \quad \text { for all } k,
$$

and $\left(\mathbf{I}_{p}[|\mu|]\right)^{\frac{q_{1}}{p-1}}\left(\mathbf{I}_{1}[|\mu|]\right)^{\frac{q_{2}}{p-1}} \in L_{l o c}^{1}\left(\mathbb{R}^{N}\right)$, thus $\chi_{B_{k}(0)}\left|u_{k}\right|^{q_{1}-1} u_{k}\left|\nabla u_{k}\right|^{q_{2}} \rightarrow|u|^{q_{1}-1} u|\nabla u|^{q_{2}}$ in $L_{l o c}^{1}\left(\mathbb{R}^{N}\right)$. This implies that, $u$ is a solution of problem (1.1) with $g(x, u, \nabla u)=|u|^{q_{1}} u|\nabla u|^{q_{2}}$ in the sense of distributions in $\mathbb{R}^{N}$ and it satisfies

$$
|u| \leq C_{0}\left(\mathbf{I}_{p}[|\mu|]\right)^{\frac{1}{p-1}}, \quad|\nabla u| \leq C_{0}\left(\mathbf{I}_{1}[|\mu|]\right)^{\frac{1}{p-1}} \text { in } B_{k}(0) .
$$

Step 2: Case $p>2$. In order to obtain the result, we will use

$$
\mathbf{W}_{\alpha, p}\left[\left(\mathbf{W}_{1, p}\left[\left|\mu_{n, k}\right|\right]\right)^{q_{1}}\left(\mathbf{W}_{\frac{1}{p}, p}\left[\left|\mu_{n, k}\right|\right]\right)^{q_{2}}\right] \leq M \mathbf{W}_{\alpha, p}\left[\left|\mu_{n, k}\right|\right]<\infty \quad \text { almost everywhere, }
$$

with $\alpha=1$ and $\alpha=1 / p$, instead of (4.4); and

$$
\mathbf{F}_{\Lambda}=\left\{u \in W_{0}^{1,1}\left(B_{2 k}(0)\right):|u| \leq \Lambda \mathbf{W}_{1, p}\left[\left|\mu_{n, k}\right|\right], \quad|\nabla u| \leq \Lambda \mathbf{W}_{\frac{1}{p}, p}\left[\left|\mu_{n, k}\right|\right] \text { in } B_{k}(0)\right\},
$$

instead of $\mathbf{E}_{\Lambda}$. We omit the details. The proof is complete.

\section{Proof of Theorem 1.2.}

Step 1: Case $\frac{3 N-2}{2 N-1}<p \leq 2$. Let $\mu_{n} \in C_{c}^{\infty}(\Omega)$ such that $\mu_{n}$ converges to $\mu$ in the sense of theorem 3.4 and $\left|\mu_{n}\right| \leq \varphi_{n} *(|\mu|)$, where $\left\{\varphi_{n}\right\}$ is a sequence of mollifiers in $\mathbb{R}^{N}$. Thanks to Proposition 2.3,

$$
\left|\mu_{n}\right|(K) \leq C^{\prime} C \operatorname{Cap}_{\mathbf{G}_{\frac{q_{1} p+q_{2}}{q_{1}+q_{2}}}, \frac{q_{1}+q_{2}}{q_{1}+q_{2}-p+1}}(K) \text { for all compact } K \subset \mathbb{R}^{N}
$$

We will prove that if $C$ in (4.9) is small enough, then for any $n \in \mathbb{N}$ the problem

$$
\begin{aligned}
-\operatorname{div}\left(A\left(x, \nabla u_{n}\right)\right) & =\left|u_{n}\right|^{q_{1}-1} u\left|\nabla u_{n}\right|^{q_{2}}+\mu_{n} & & \text { in } \Omega \\
u_{n} & =0 & & \text { on } \Omega,
\end{aligned}
$$


has a renormalized solution satisfying

$$
\left|u_{n}\right| \leq C_{0}\left(\mathbf{I}_{p}^{4 R}\left[\left|\mu_{n}\right|\right]\right)^{\frac{1}{p-1}}, \quad\left|\nabla u_{n}\right| \leq C_{0}\left(\mathbf{I}_{1}^{4 R}\left[\left|\mu_{n}\right|\right]\right)^{\frac{1}{p-1}} \text { in } \Omega .
$$

By Theorem (2.12), we need to prove that there exists $M>0$ such that, if for $\alpha=1$ and $p$, the following inequalities hold,

$$
\mathbf{I}_{\alpha}\left[\left(\mathbf{I}_{p}^{4 R}\left[\left|\mu_{n, k}\right|\right]\right)^{\frac{q_{1}}{p-1}}\left(\mathbf{I}_{1}^{4 R}\left[\left[\left|\mu_{n, k}\right|\right]\right)^{\frac{q_{2}}{p-1}}\right] \leq M \mathbf{I}_{\alpha}^{4 R}\left[\left|\mu_{n, k}\right|\right]<\infty \quad\right. \text { almost everywhere, }
$$

then problem (4.10) has a renormalized solution satisfying (4.11).

We have to prove that there exists $M>0$ such that if $\alpha=1$ and $p$, there holds

$$
\mathbf{I}_{\alpha}^{4 R}\left[\left(\mathbf{I}_{p}^{4 R}\left[\left|\mu_{n}\right|\right]\right)^{\frac{q_{1}}{p-1}}\left(\mathbf{I}_{1}^{4 R}\left[\left|\mu_{n}\right|\right]\right)^{\frac{q_{2}}{p-1}}\right] \leq M \mathbf{I}_{\alpha}^{4 R}\left[\left|\mu_{n}\right|\right]<\infty \quad \text { almost everywhere in } B_{2 R}\left(x_{0}\right),
$$

For $n \in \mathbb{N}$ fixed, we set

$$
\mathbf{E}_{\Lambda}=\left\{u \in W_{0}^{1,1}(\Omega):|u| \leq \Lambda\left(\mathbf{I}_{p}^{4 R}\left[\left|\mu_{n}\right|\right]\right)^{\frac{1}{p-1}},|\nabla u| \leq \Lambda\left(\mathbf{I}_{1}^{4 R}\left[\left|\mu_{n}\right|\right]\right)^{\frac{1}{p-1}} \text { in } \Omega\right\} .
$$

Clearly, $\mathbf{E}_{\Lambda}$ is closed under the strong topology of $W_{0}^{1,1}(\Omega)$, convex and $|u|^{q_{1}}|\nabla u|^{q_{2}} \in L^{\infty}(\Omega)$ for any $u \in \mathbf{E}_{\Lambda}$. We consider the map $S: \mathbf{E}_{\Lambda} \mapsto W_{0}^{1,1}(\Omega)$ defined for each $v \in \mathbf{E}_{\Lambda}$ by $S(v)=u$, where $u \in W_{0}^{1,1}(\Omega)$ is the unique renormalized solution of

$$
\begin{aligned}
-\operatorname{div}(A(x, \nabla u)) & =|v|^{q_{1}-1} v|\nabla v|^{q_{2}}+\mu_{n} \text { in } \Omega \\
u & =0 \quad \text { on } \partial \Omega,
\end{aligned}
$$

We will show that $S\left(\mathbf{E}_{\Lambda}\right)$ is subset of $\mathbf{E}_{\Lambda}$ for some $\Lambda>0$ small enough.

For $v \in \mathbf{E}_{\Lambda}$ and $u=S(v)$, we have

$$
|v| \leq \Lambda\left(\mathbf{I}_{p}^{4 R}\left[\left|\mu_{n}\right|\right]\right)^{\frac{1}{p-1}},|\nabla v| \leq \Lambda\left(\mathbf{I}_{1}^{4 R}\left[|\mu|_{n}\right]\right)^{\frac{1}{p-1}} .
$$

In particular,

$$
\left\|\left.v\right|_{L^{\infty}\left(\Omega_{d / 2}\right)} \leq C_{1} \Lambda d^{-\frac{N-p}{p-1}}\left(\left|\mu_{n}\right|(\Omega)\right)^{1 /(p-1)}, \quad\right\| \nabla v \|_{L^{\infty}\left(\Omega_{d / 2}\right)} \leq C_{1} \Lambda d^{-\frac{N-1}{p-1}}\left(\left|\mu_{n}\right|(\Omega)\right)^{1 /(p-1)},
$$

where $\Omega_{d / 2}=\{x \in \Omega: d(x, \partial \Omega) \leq d / 2\}$.

From (4.13) with $\alpha=1$ and $p$ we derive

$$
\begin{aligned}
\mathbf{I}_{p}^{4 R}\left[\left.|| v\right|^{q_{1}-1} v|\nabla v|^{q_{2}}+\mu_{n} \mid\right] & \leq \Lambda^{q_{1}+q_{2}} \mathbf{I}_{p}^{4 R}\left[\left(\mathbf{I}_{p}^{4 R}\left[\left|\mu_{n}\right|\right]\right)^{\frac{q_{1}}{p-1}}\left(\mathbf{I}_{1}^{4 R}\left[\left|\mu_{n}\right|\right]\right)^{\frac{q_{2}}{p-1}}\right]+\mathbf{I}_{p}^{4 R}\left[\left|\mu_{n}\right|\right] \\
& \leq\left(\Lambda^{q_{1}+q_{2}} M+1\right) \mathbf{I}_{p}^{4 R}\left[\left|\mu_{n}\right|\right],
\end{aligned}
$$

and

$$
\begin{aligned}
\mathbf{I}_{1}^{4 R}\left[\left.|| v\right|^{q_{1}-1} v|\nabla v|^{q_{2}}+\mu_{n} \mid\right] & \leq \Lambda^{q_{1}+q_{2}} \mathbf{I}_{1}^{4 R}\left[\left(\mathbf{I}_{p}^{4 R}[|\mu|]\right)^{\frac{q_{1}}{p-1}}\left(\mathbf{I}_{1}^{4 R}\left[\left|\mu_{n}\right|\right]\right)^{\frac{q_{2}}{p-1}}\right]+\mathbf{I}_{1}^{4 R}\left[\left|\mu_{n}\right|\right] \\
& \leq\left(\Lambda^{q_{1}+q_{2}} M+1\right) \mathbf{I}_{1}^{4 R}\left[\left|\mu_{n}\right|\right] .
\end{aligned}
$$

By Theorem 3.6 and $\mathbf{W}_{1, p}^{4 R}\left[\left|\mu_{n}\right|\right] \lesssim\left(\mathbf{I}_{p}^{4 R}\left[\left|\mu_{n}\right|\right]\right)^{\frac{1}{p-1}}$ in $\Omega$, we have

$$
\begin{aligned}
|u| & \leq C_{2}\left(\mathbf{I}_{p}^{4 R}\left[\left.|| v\right|^{q_{1}-1} v|\nabla v|^{q_{2}}+\mu_{n} \mid\right]\right)^{\frac{1}{p-1}} \\
& \leq C_{2}\left(\Lambda^{q_{1}+q_{2}} M+1\right)^{\frac{1}{p-1}}\left(\mathbf{I}_{p}^{4 R}\left[\left|\mu_{n}\right|\right]\right)^{\frac{1}{p-1}} \quad \text { in } \Omega .
\end{aligned}
$$


From Corollary 3.8, we derive

$$
\begin{aligned}
|\nabla u(x)| & \leq C_{3}\left(\frac{R}{d}\right)^{N}\left(\mathbf{I}_{1}^{4 R}\left[\left.|| v\right|^{q_{1}-1} v|\nabla v|^{q_{2}}+\mu_{n} \mid\right](x)\right)^{\frac{1}{p-1}} \\
& \leq C_{4}\left(\Lambda^{q_{1}+q_{2}} M+1\right)^{\frac{1}{p-1}}\left(\mathbf{I}_{1}^{4 R}\left[\left|\mu_{n}\right|\right](x)\right)^{\frac{1}{p-1}}
\end{aligned}
$$

for any $x \in \Omega$ verifying $d(x, \partial \Omega)>d / 4$. By the standard regularity results for quasilinear equations, we deduce

$$
\|\mid \nabla u\|_{L^{\infty}\left(\Omega_{d / 4}\right)} \leq C_{5}\left(\|u\|_{L^{\infty}\left(\Omega_{d / 2}\right)}+|||v|^{q_{1}}|\nabla v|^{q_{2}} \|_{L^{\infty}\left(\Omega_{d / 2}\right)}^{1 /(p-1)}\right),
$$

where $C_{5}=C_{5}(N, p, \Omega)$.

(a) Estimate of $\left.|||v|^{q_{1}}|\nabla v|^{q_{2}}\right|_{L^{\infty}\left(\Omega_{d / 2}\right)} ^{1 /(p-1)}$. From (4.13), we have $\left|\mu_{n}\right|(\Omega) \leq C_{6} M^{\frac{p-1}{q_{1}+q_{2}-p+1}}$. Thus

$$
\begin{aligned}
\left\||v|^{q_{1}}|\nabla v|^{q_{2}}\right\|_{L^{\infty}\left(\Omega_{d / 2}\right)}^{1 /(p-1)} & \leq\|v\|_{L^{\infty}\left(\Omega_{d / 2}\right)}^{\frac{q_{1}}{p-1}}|| \nabla v \mid \|_{L^{\infty}\left(\Omega_{d / 2}\right)}^{\frac{q_{2}}{p-1}} \\
& \leq\left(C_{1} \Lambda d^{-\frac{N-p}{p-1}}\left(\left|\mu_{n}\right|(\Omega)\right)^{1 /(p-1)}\right)^{\frac{q_{1}}{p-1}}\left(C_{1} \Lambda d^{-\frac{N-1}{p-1}}\left(\left|\mu_{n}\right|(\Omega)\right)^{1 /(p-1)}\right)^{\frac{q_{2}}{p-1}} \\
& \leq C_{7} \Lambda^{\frac{q_{1}}{p-1}+\frac{q_{2}}{p-1}}\left(\left|\mu_{n}\right|(\Omega)\right)^{\frac{q_{1}+q_{2}}{(p-1)^{2}}} \\
& \leq C_{8} \Lambda^{\frac{q_{1}}{p-1}+\frac{q_{2}}{p-1}} M^{\frac{1}{p-1}} \inf _{x \in \Omega}\left(\mathbf{I}_{1}^{4 R}\left[\left|\mu_{n}\right|\right](x)\right)^{\frac{1}{p-1}}
\end{aligned}
$$

where $C_{8}=C_{8}\left(N, p, \alpha, q_{1}, q_{2}, d, R\right)$.

(b) Estimate of $\|u\|_{L^{\infty}\left(\Omega_{d / 2}\right)}$. By (4.14) we have

$$
\begin{aligned}
\|u\|_{L^{\infty}\left(\Omega_{d / 2}\right)} & \leq C_{2}\left(\Lambda^{q_{1}+q_{2}} M+1\right)^{\frac{1}{p-1}}\left(\left\|\mathbf{I}_{p}^{4 R}\left[\left|\mu_{n}\right|\right]\right\|_{L^{\infty}\left(\Omega_{d / 2}\right)}\right)^{\frac{1}{p-1}} \\
& \leq C_{9}\left(\Lambda^{q_{1}+q_{2}} M+1\right)^{\frac{1}{p-1}} d^{-\frac{N-p}{p-1}}\left(\left|\mu_{n}\right|(\Omega)\right)^{\frac{1}{p-1}} \\
& \leq C_{10}\left(\Lambda^{q_{1}+q_{2}} M+1\right)^{\frac{1}{p-1}} \inf _{x \in \Omega}\left(\mathbf{I}_{1}^{4 R}\left[\left|\mu_{n}\right|\right](x)\right)^{\frac{1}{p-1}} .
\end{aligned}
$$

Therefore,

$$
\|\mid \nabla u\|_{L^{\infty}\left(\Omega_{d / 4}\right)} \leq C_{11}\left(\Lambda^{\frac{q_{1}}{p-1}+\frac{q_{2}}{p-1}} M^{\frac{1}{p-1}}+\left(\Lambda^{q_{1}+q_{2}} M+1\right)^{\frac{1}{p-1}}\right) \inf _{x \in \Omega}\left(\mathbf{I}_{1}^{4 R}\left[\left|\mu_{n}\right|\right](x)\right)^{\frac{1}{p-1}}
$$

where $C_{11}=C_{11}\left(N, p, \alpha, q_{1}, q_{2}, d, R, \Omega\right)$.

Combining this with (4.15) we get for all $x \in \Omega$,

$$
\begin{aligned}
|\nabla u(x)| & \leq C_{4}\left(\Lambda^{q_{1}+q_{2}} M+1\right)^{\frac{1}{p-1}}\left(\mathbf{I}_{1}^{4 R}\left[\left|\mu_{n}\right|\right](x)\right)^{\frac{1}{p-1}} \\
& +C_{11}\left(\Lambda^{\frac{q_{1}}{p-1}+\frac{q_{2}}{p-1}} M^{\frac{1}{p-1}}+\left(\Lambda^{q_{1}+q_{2}} M+1\right)^{\frac{1}{p-1}}\right)\left(\mathbf{I}_{1}^{4 R}\left[\left|\mu_{n}\right|\right](x)\right)^{\frac{1}{p-1}} .
\end{aligned}
$$

We can find $M, \Lambda>0$ such that

$$
\begin{aligned}
& C_{2}\left(\Lambda^{q_{1}+q_{2}} M+1\right)^{\frac{1}{p-1}} \leq \Lambda, \\
& C_{4}\left(\Lambda^{q_{1}+q_{2}} M+1\right)^{\frac{1}{p-1}}+C_{11}\left(\Lambda^{\frac{q_{1}}{p-1}+\frac{q_{2}}{p-1}} M^{\frac{1}{p-1}}+\left(\Lambda^{q_{1}+q_{2}} M+1\right)^{\frac{1}{p-1}}\right) \leq \Lambda .
\end{aligned}
$$


Thus, from (4.14) and (4.16) we obtain $S\left(\mathbf{E}_{\Lambda}\right) \subset \mathbf{E}_{\Lambda}$. Moreover, it can be shown that the $\operatorname{map} S: \mathbf{E}_{\Lambda} \mapsto \mathbf{E}_{\Lambda}$ is continuous and $S\left(\mathbf{E}_{\Lambda}\right)$ is pre-compact under the strong topology of $W_{0}^{1,1}(\Omega)$. Then by Schauder Fixed Point Theorem, $S$ has a fixed point on $\mathbf{E}_{\Lambda}$. This means problem (4.10) has a renormalized solution satisfying (4.11).

Step 2: The case $p>2$. To obtain the result, we will use

$$
\mathbf{W}_{\alpha, p}^{4 R}\left[\left(\mathbf{W}_{1, p}^{4 R}\left[\left|\mu_{n}\right|\right]\right)^{q_{1}}\left(\mathbf{W}_{\frac{1}{p}, p}\left[\left|\mu_{n}\right|\right]\right)^{q_{2}}\right] \leq M \mathbf{W}_{\alpha, p}^{4 R}[|\mu|]<\infty \quad \text { almost everywhere in } \Omega
$$

with $\alpha=1$ and $\alpha=1 / p$, instead of (4.4); and

$$
\mathbf{F}_{\Lambda}=\left\{u \in W_{0}^{1,1}(\Omega):|u| \leq \Lambda \mathbf{W}_{1, p}^{4 R}\left[\left|\mu_{n}\right|\right], \quad|\nabla u| \leq \Lambda \mathbf{W}_{\frac{1}{p}, p}^{4 R}\left[\left|\mu_{n}\right|\right] \text { in } \Omega\right\}
$$

instead of $\mathbf{E}_{\Lambda}$. We omit the details. The proof is complete.

\section{References}

[1] D. R. Adams, L.I. Hedberg, Function Spaces and Potential Theory, Grundlehren der Mathematischen Wisenschaften 31, Springer-Verlag (1999).

[2] D. R. Adams, On the existence of capacitary strong type estimates in $\mathbb{R}^{N}$, Ark. Mat. 14 (1976), 125-140.

[3] L. Ambrosio, N.Fusco, D.Pallara, Functions of bounded variation and free discontinuity problems, Oxford University Press, 2000.

[4] M. F. Bidaut-Véron, Local and global behavior of solutions of quasilinear equations of Emden-Fowler type, Arch. Ration. Mech. Anal. 107 (1989), 293-324.

[5] M. F. Bidaut-Véron, Necessary conditions of existence for an elliptic equation with source term and measure data involving p-Laplacian, in: Proc. 2001 Luminy Conf. on Quasilinear Elliptic and Parabolic Equations and Systems, Electron. J. Differ. Equ. Conf. 8 (2002), 23-34.

[6] M. F. Bidaut-Véron, Removable singularities and existence for a quasilinear equation with absorption or source term and measure data, Adv. Nonlinear Stud. 3 (2003), 25-63.

[7] M. F. Bidaut-Véron, Q. H. Nguyen, L. Véron, Quasilinear Lane-Emden equations with absorption and measure data, J. Math. Pures Appl. 102 (2014), 315-337 .

[8] M. F. Bidaut-Véron, Q. H. Nguyen, L. Véron, Quasilinear and Hessian LaneEmden systems with reaction and measure data, Potential Anal., to appear, doi.org/10.1007/s11118-018-9753-z.

[9] G. Dal Maso, F. Murat, L. Orsina, A. Prignet, Renormalized solutions of elliptic equations with general measure data, Ann. Sc. Norm. Sup. Pisa, 28 (1999), 741-808.

[10] F. Duzaar, G. Mingione, Gradient estimates via linear and nonlinear potential, J. Funct. Anal. 259 (2010), 2961-2998.

[11] R. Fefferman, Strong differentiation with respect to measure, Amer. J. Math 103 (1981), $33-40$.

[12] P. Honzik and B. Jaye, On the good- $\lambda$ inequality for nonlinear potentials, Proc. Amer. Math. Soc. 140 (2012), 4167-4180.

[13] N.J. Kalton, I.E. Verbitsky, Nonlinear equations and weighted norm inequality, Trans. Amer. Math. Soc. 351 (1999), 3441-3497. 
[14] T. Kuusi, G. Mingione, Linear potential in nonlinear potential theory, Arch. Rat. Mech. Anal. 207 (2013), 207-246.

[15] Q. H. Nguyen, Potential estimates and quasilinear parabolic equations with measure data, arXiv:1405.2587, pp. 1-120, submitted

[16] Q.-H. Nguyen and N. C. Phuc, Good- $\lambda$ and Muckenhoupt-Wheeden type bounds in quasilinear measure datum problems, with applications, Math. Ann. 374 (2019), 67-98.

[17] Q.-H. Nguyen and N. C. Phuc, Pointwise gradient estimates for a class of singular quasilinear equation with measure data. J. Funct. Anal. 278 (2020) Issue 5, 108391.

[18] Q.-H. Nguyen and N. C. Phuc, Existence and regularity estimates for quasilinear equations with measure data: the case $1<p \leq \frac{3 n-2}{2 n-1}$. Submitted for publication.

[19] Q.-H. Nguyen and N. C. Phuc, Quasilinear Riccati type equations with oscillatory and singular data. to appear in Advanced Nonlinear Studies, (arXiv:2003.03724).

[20] Q. H. Nguyen, L. Véron, Quasilinear and Hessian Type Equations with Exponential Reaction and Measure Data, Arch. Rat. Mech. Anal. 214 (2014), 235-267.

[21] V. G. Maz'ya, I. E. Verbitsky, Capacitary inequalities for fractional integrals, with applications to partial differential equations and sobolev multipliers, Arkiv Mat, 33 (1995), 81-115.

[22] N. C. Phuc, I. E. Verbitsky, Quasilinear and Hessian equations of Lane-Emden type, Ann. Math. 168 (2008), 859-914 (2008).

[23] N. C. Phuc, Quasilinear Riccati type equations with super-critical exponents, Comm. Part. Diff. Equ. 35 (2010), 1958-1981.

[24] N. C. Phuc, Nonlinear Muckenhoupt-Wheeden type bounds on Reifenberg flat domains, with application to quasilinear Riccati type equations, Adv. in Math. 250 (2014), 378419

[25] N. C. Phuc, I. E. Verbitsky, Singular quasilinear and Hessian equation and inequalities, J. Funct Anal. 256 (2009), 1875-1906.

[26] N. S. Trudinger, X. J. Wang, Quasilinear elliptic equations with signed measure data, Discr. Cont. Dyn. Syst. 23 (2009), 477-494.

[27] L. Véron, Local and Global Aspects of Quasilinear Degenerate Elliptic Equations, World Scientific Publishing Co. Pte. Ltd., Hackensack, NJ (2017). 